\title{
Dynamic Element Matching Techniques For Delta-Sigma ADCs With Large Internal Quantizers
}

\author{
Brent C. Nordick \\ Brigham Young University - Provo
}

Follow this and additional works at: https://scholarsarchive.byu.edu/etd

Part of the Electrical and Computer Engineering Commons

\section{BYU ScholarsArchive Citation}

Nordick, Brent C., "Dynamic Element Matching Techniques For Delta-Sigma ADCs With Large Internal Quantizers" (2004). Theses and Dissertations. 134.

https://scholarsarchive.byu.edu/etd/134

This Thesis is brought to you for free and open access by BYU ScholarsArchive. It has been accepted for inclusion in Theses and Dissertations by an authorized administrator of BYU ScholarsArchive. For more information, please contact scholarsarchive@byu.edu, ellen_amatangelo@byu.edu. 


\section{DYNAMIC ELEMENT MATCHING TECHNIQUES FOR DELTA-SIGMA ADCS WITH LARGE INTERNAL QUANTIZERS}

by

Brent C. Nordick

A thesis submitted to the faculty of

Brigham Young University

in partial fulfillment of the requirements for the degree of

Master of Science

Department of Electrical and Computer Engineering

Brigham Young University

August 2004 
Copyright (C) 2004 Brent C. Nordick

All Rights Reserved 


\begin{abstract}
DYNAMIC ELEMENT MATCHING TECHNIQUES FOR DELTA-SIGMA ADCS WITH LARGE INTERNAL QUANTIZERS
\end{abstract}

\author{
Brent C. Nordick \\ Department of Electrical and Computer Engineering \\ Master of Science
}

This thesis presents two methods that enable high internal quantizer resolution in delta-sigma analog-to-digital converters. Increasing the quantizer resolution in a delta-sigma modulator can increase SNR, improve stability and reduce integrator power consumption. However, each added bit of quantizer resolution also causes an exponential increase in the power dissipation, required area and complexity of the dynamic element matching (DEM) circuit required to attenuate digital-to-analog converter (DAC) mismatch errors. One way to overcome these drawbacks is to segment the feedback signal, creating a "coarse" signal and a "fine" signal. This reduces the DEM circuit complexity, power dissipation, and size. However, it also creates additional problems. The negative consequences of segmentation are presented, along with two potential solutions: one that uses calibration to cancel mismatch between the "coarse" DAC and the "fine" DAC, and another that frequency-shapes this mismatch error. Mathematical analysis and behavioral simulation results are presented. A potential circuit design for the frequency-shaping method is presented in detail. Circuit simulations for one of the proposed implementations show that the delay through the digital path is under $7 \mathrm{~ns}$, thus permitting a $50 \mathrm{MHz}$ clock frequency for the overall ADC. 


\section{ACKNOWLEDGMENTS}

I would like to gratefully acknowledge the support and encouragement from my wife, Andrea. To her go the official titles of "Tech Writer" and "Hyphenation-Queen" for all the proofreading and grammar checking. (Any mistakes still present in this text are most likely because I made some changes and didn't ask her to check them over.)

Special thanks to my wonderful parents for their support, and for providing a roof over our heads for a bit when it was needed. Thanks as well to my brothers and sisters for their examples in striving for advanced degrees.

I would like to acknowledge Dr. Craig Petrie for the time he has taken to proofread and suggest improvements for the various drafts of this thesis. I also acknowledge the other two members of my graduate committee, Dr. Michael Rice and Dr. Brent Nelson, for their help with my thesis. Special thanks to all three for the instruction and mentoring they have provided during my time as a student at BYU.

I would like to thank the Electrical Engineering Department of BYU: the combination of wonderful professors, staff, and laboratory facilities made all the difference. (Additional thanks for the $\mathrm{HT}_{\mathrm{E}} \mathrm{X}$ thesis style package they provided.)

Special thanks to the Intel Research Council for funding this research. 


\section{Contents}

$\begin{array}{ll}\text { Acknowledgments vi } & \text { vi }\end{array}$

List of Tables $\quad x$

List of Figures $\quad$ xii

\begin{tabular}{lll}
\hline Introduction & 1
\end{tabular}

1.1 Thesis Overview . . . . . . . . . . . . . . . . . . . . . . . . . . 2

\begin{tabular}{|lll}
\hline 2 & Delta-Sigma ADCs & 3
\end{tabular}

2.1 Introduction to Delta-Sigma ADCs . . . . . . . . . . . . . . . . 3

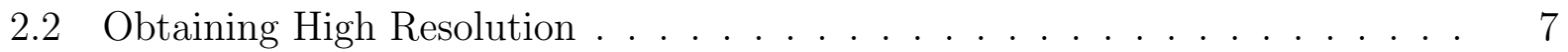

2.3 Multi-Bit Internal Quantization . . . . . . . . . . . . . . . . . . . 9

2.4 High Internal Quantization . . . . . . . . . . . . . . . . . . . . . 12

2.5 Conclusion . . . . . . . . . . . . . . . . . . . . . . . . 13

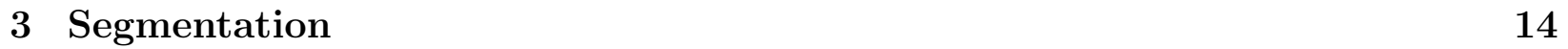

3.1 Segmenting the Digital Word . . . . . . . . . . . . . . . . . . . . 14

3.2 Analysis of the Problem . . . . . . . . . . . . . . . . . . 17

3.3 Conclusion . . . . . . . . . . . . . . . . . . . . . . . . . 18

\begin{tabular}{lll}
\hline Calibration & 19
\end{tabular}

4.1 The Motivation For Calibration . . . . . . . . . . . . . . . . . . . . . . 19

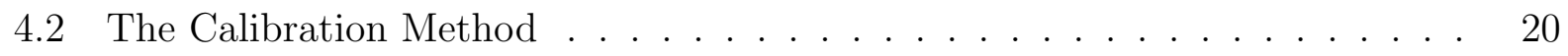

4.3 Drawbacks and Benefits . . . . . . . . . . . . . . . . . . . . . 22

4.4 Behavioral Simulation Results . . . . . . . . . . . . . . . . . . . . . . . . 22 
4.5 Conclusion $\ldots \ldots \ldots \ldots \ldots$

$\begin{array}{lll}5 & \text { Requantization } & 25\end{array}$

5.1 The Noise-Shaped Requantization (ReQ) Method . . . . . . . . . . . . . . . 25

5.2 Drawbacks and Benefits . . . . . . . . . . . . . . . . . . . . 27

5.3 Behavioral Circuit Simulation Results . . . . . . . . . . . . . . . . . . 31

5.4 Conclusion $\ldots \ldots \ldots \ldots \ldots$

6 Circuit Design 33

6.1 Circuit Overviews . . . . . . . . . . . . . . . . . . . . . . . 33

6.1 .1 Encoder $\ldots \ldots \ldots \ldots \ldots$

6.1 .2 ReQ Block . . . . . . . . . . . . . . . . . . . . . . . . . . . . . . 34

$6.1 .3 \quad$ Decode Logic $\ldots \ldots \ldots$

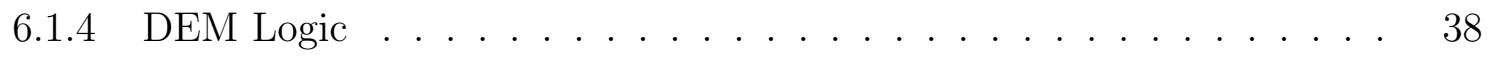

6.2 Circuit Simulations . . . . . . . . . . . . . . . . . . . . . . 45

6.3 Conclusion . . . . . . . . . . . . . . . . . . . . . . . . 47

$\begin{array}{lll}7 & \text { Conclusion } & 53\end{array}$

7.1 Contributions . . . . . . . . . . . . . . . . . . . . 53

7.2 Summary of Results $\ldots \ldots \ldots \ldots \ldots$

7.3 Areas for Potential Future Research . . . . . . . . . . . . . . . . . 54

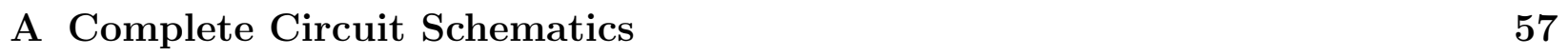

A.1 Top Level Schematic . . . . . . . . . . . . . . . . . . . . . . . . 57

A.2 Bubble Decode . . . . . . . . . . . . . . . . . . . . . . 58

A.2.1 Inverter . . . . . . . . . . . . . . . . . . . . . . . . . . . . . . . 59

A.2.2 3 Input Nor . . . . . . . . . . . . . . . . . . . . . 60

A.3 2's Complement Encoder . . . . . . . . . . . . . . . . . . . . . . . . . 61

A.4 Unsigned Encoder . . . . . . . . . . . . . . . . . . . . . . . . . . . . . 62

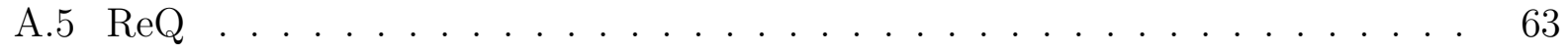

A.5.1 8-bit Subtractor . . . . . . . . . . . . . . . . . . . . 64

A.5.2 Full Adder . . . . . . . . . . . . . . . . . . . . . . . 65 
A.5.3 5-bit Subtractor . . . . . . . . . . . . . . . . . . . . 66

A.5.4 D-Register Bank . . . . . . . . . . . . . . . . . 67

A.5.5 D-Register . . . . . . . . . . . . . . . . . . . . 68

A.5.6 D-Latch . . . . . . . . . . . . . . . . . . . . . . . . . . . . 69

A.6 4-bit Decoder Logic . . . . . . . . . . . . . . . . . . . . . . . 70

A.7 5-bit Decoder Logic . . . . . . . . . . . . . . . . . . . . . . . . . 71

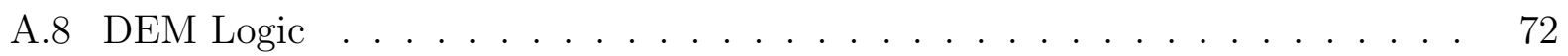

A.8.1 16-line Rotate-1 Logic . . . . . . . . . . . . . . . . . . . . 73

A.8.2 31-line Rotate-1 Logic . . . . . . . . . . . . . . . . . . . . 74

A.8.3 2-to-1 Mux . . . . . . . . . . . . . . . . . . . 75

\begin{tabular}{ll}
\hline B MATLAB Code & 76
\end{tabular}

B.1 Reference Case Code . . . . . . . . . . . . . . . . . . . . . . 76

B.2 Calibration Code . . . . . . . . . . . . . . . . . . . . . 80

B.3 ReQ Code . . . . . . . . . . . . . . . . . . . . . . . . . . . . . . . . . . 88

B.4 Manual Calibration Code. . . . . . . . . . . . . . . . . . . . . . . . . 94

B.5 Additional Functions . . . . . . . . . . . . . . . . . . . . . . . . . 101

B.5.1 mypsd . . . . . . . . . . . . . . . . . . . . . . . . 101

B.5.2 mysnr . . . . . . . . . . . . . . . . . . . . . 102

\begin{tabular}{lr}
\hline Bibliography & 104
\end{tabular} 


\section{List of Tables}

2.1 Different Types of DACs . . . . . . . . . . . . . . . . . . . 11

2.2 Example Operation of Different Types of DACs . . . . . . . . . . . . . . 11

4.1 Simulated SNR Results for Segmented $\Delta \Sigma$ ADC (in dB) . . . . . . . . . . . 20

6.1 Logic Table for 4-Bit Binary-to-Thermometer Code Conversion. . . . . . . . 44

6.2 Timing Results for the Critical Path . . . . . . . . . . . . . . . . . 47 


\section{List of Figures}

2.1 Comparison Between a Nyquist One-Bit ADC and a One-Bit $\Delta \Sigma \mathrm{ADC}$. . . 4

2.2 Circuit Level Block Diagram of a First-Order One-Bit $\Delta \Sigma \mathrm{ADC} \ldots \ldots$

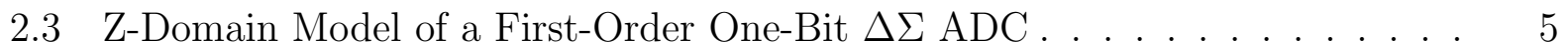

$2.4 \quad$ Frequency Plot Showing $S_{Q}\left(e^{j w}\right)$ and $S_{N}\left(e^{j w}\right)$ for a $\Delta \Sigma$ ADC $\ldots \ldots . .8$

$2.5 \quad$ Basic First-Order $\Delta \Sigma$ ADC Block Diagram $\ldots \ldots \ldots \ldots \ldots$

2.6 Graphical Explanation of DEM Operation . . . . . . . . . . . . . . . . 12

3.1 Block Diagram: $\Delta \Sigma$ ADC With a Segmented DAC/DEM Structure … . 15

3.2 Mathematical Block Diagram of a Segmented $\Delta \Sigma$ ADC $\ldots \ldots \ldots \ldots$

$3.3 \quad$ Simulation Results: 2nd-Order $\Delta \Sigma$ ADC With Segmented Feedback $\ldots .$.

4.1 Block Diagram of Calibration Method . . . . . . . . . . . . . . . . . . 21

4.2 Simulated Results for Calibrated System . . . . . . . . . . . . . . . . . 23

$5.1 \quad$ Mathematical Block Diagram of ReQ Architecture . . . . . . . . . . . . 26

5.2 Power Spectra Showing DC Offset in Ideal ReQ Simulation . . . . . . . . . . 27

5.3 Simulated Results for ReQ System … . . . . . . . . . . . . . . . . 32

6.1 Overall Block Diagram of the Feedback Path for the ReQ Method . . . . . . 34

6.2 Bubble Decode Circuitry . . . . . . . . . . . . . . . . . . . . . . . . . . . . 35

6.3 Encoder Circuit for the MSBs . . . . . . . . . . . . . . . . 36

6.4 Encoder Circuit for the LSBs $\ldots \ldots \ldots \ldots$

$6.5 \quad \operatorname{ReQ}$ Circuit $\ldots \ldots \ldots \ldots \ldots$

6.6 Full Adder Circuit . . . . . . . . . . . . . . . . . . . . . . . 40

6.7 D-Latch Circuit . . . . . . . . . . . . . . . . . . . . . . . . 41

6.8 D-Register Circuit . . . . . . . . . . . . . . . . . . . . . . . . . . . . . . 41

6.9 4-Bit Binary-to-Thermometer Decoder Circuit . . . . . . . . . . . . . . 42

6.105 -Bit Binary-to-Thermometer Decoder Circuit . . . . . . . . . . . . . . 43 
6.11 Block Diagram of DEM Circuitry $\ldots \ldots \ldots$. . . . . . . . . . 45

6.12 DEM Circuitry $\ldots \ldots \ldots \ldots$

6.13 Timing Simulation for ReQ Bubble Decode Block . . . . . . . . . . . . . . 48

6.14 Timing Simulation for ReQ Encoder Block . . . . . . . . . . . . . . . . 49

6.15 Timing Simulation for ReQ 8-bit Subtractor Block. . . . . . . . . . . . 50

6.16 Timing Simulation for ReQ 5-bit Subtractor Block . . . . . . . . . . . . . 51

6.17 Timing Simulation for ReQ Decoder Block . . . . . . . . . . . . . . . 52

A.1 Overall Schematic for Digital Feedback Path . . . . . . . . . . . . 57

A.2 Bubble Decode Logic . . . . . . . . . . . . . . . . . . . . . . . . 58

A.3 Inverter . . . . . . . . . . . . . . . . . . . . . . . . . . . . 59

A.4 Nor Gate . . . . . . . . . . . . . . . . . . . . . . . . . . . . . . . . 60

A.5 2's Complement Encoder . . . . . . . . . . . . . . . . . . . . . . 61

A.6 Unsigned Encoder . . . . . . . . . . . . . . . . . . . . . . . . . . . . . . . . 62

A.7 Requantization Logic . . . . . . . . . . . . . . . . . . . . . . . . . 63

A.8 8-bit Subtractor . . . . . . . . . . . . . . . . . . . . . . . . . . . . 64

A.9 Full Adder Design . . . . . . . . . . . . . . . . . . . . . . . . . 65

A.105-bit Subtractor . . . . . . . . . . . . . . . . . . . 66

A.11 D-Register Bank . . . . . . . . . . . . . . . . . . . . . 67

A.12 D-Register . . . . . . . . . . . . . . . . . . . . . . . 68

A.13 D-Latch . . . . . . . . . . . . . . . . . . . . . . . 69

A.14 4-bit Binary-to-Thermometer Code Decoder ．.. . . . . . . . . . . . . 70

A.15 5-bit Binary-to-Thermometer Code Decoder . . . . . . . . . . . . . 71

A.16 Dynamic Element Matching Logic . . . . . . . . . . . . . . . . . . . 72

A.17 16-Input Rotate-1 . . . . . . . . . . . . . . . . . . . . . 73

A.18 31-Input Rotate-1 . . . . . . . . . . . . . . . . . . . . . . . 74

A.192-to-1 Mux . . . . . . . . . . . . . . . . . . . . . 75 


\section{Chapter 1}

\section{Introduction}

The analog-to-digital converter (ADC) is the essential link between "real world" analog signals and digital electronics. In the current digital age, they are nearly everywhere: car sensors, home thermostats, DVD and CD players, televisions, personal computers, cell phones, and many other common devices. As consumers expect more and more from digital electronic devices, faster ADCs with higher resolution are needed. At the same time, cell phones and other "mobile" technologies are demanding lower power alternatives. As such, improving present $\mathrm{ADC}$ architectures is an active field of research.

The delta-sigma $(\Delta \Sigma)$ ADC is one architecture that is being examined for use in lowpower, high-resolution, moderate-speed applications. Conventionally, $\Delta \Sigma$ ADCs are used for low-frequency applications $(<100 \mathrm{kHz})$ requiring high resolution $(>14 \mathrm{bits})$, such as

digital audio or high-precision instrumentation [1, 2, 3. Recent work, however, is extending the signal bandwidths of $\Delta \Sigma$ ADCs into the $\mathrm{MHz}$ range while maintaining high resolution [4, 5].

One method of extending the signal bandwidth of a $\Delta \Sigma \mathrm{ADC}$ without decreasing the resolution is to appropriately trade off internal quantization and oversampling ratio. As the oversampling ratio is reduced, the signal bandwidth increases, but the signal-to-noise ratio (resolution) is decreased. This can be recovered by increasing the number of bits in the internal quantization path. Several problems arise from this approach. This thesis presents an analysis of these problems and two potential solutions. Simulations show that near-ideal resolution can be maintained for practical implementations. 


\subsection{Thesis Overview}

The thesis begins with a short introduction of $\Delta \Sigma$ ADCs (Chapter 2). Some of the issues involved in designing high-speed, high-resolution ADCs are presented. Increasing the resolution of the internal quantization is shown to be a desirable step for higher bandwidth design, and accompanying problems for quantization levels above one bit are discussed. Problems that occur when internal quantization levels are increased too far are covered, and a potential solution is presented: segmentation.

In Chapter 3, the proposed method of segmentation is described, along with its inherent drawbacks. The method is analyzed, and the source of the main drawback is identified.

In Chapter 4, calibration is presented as a potential solution to the problems associated with segmentation. A mathematical analysis is provided. An in-depth description of how to implement the calibration solution is presented, along with the potential benefits and drawbacks. Behavioral simulations are provided to demonstrate the performance of this solution.

In Chapter 5, another potential solution is presented, this one adapting a method of selecting the coarse and fine bits developed for $\Delta \Sigma$ DACs [6]. Again, a mathematical analysis of this solution is presented. A description of the required hardware and its operation is provided, again with the potential benefits and drawbacks. Simulation results are shown to demonstrate this solution's performance.

In Chapter 6, circuits implementing the ReQ method are presented, with schematics

and explanations. SPICE simulations are presented showing the worst-case timing of the design, verifying that it is fast enough to meet the design constraints.

As a conclusion, Chapter 7 reviews the research presented and then compares the results of the two potential solutions and their respective strengths and weaknesses. Contributions of this project are presented, and areas for continued research are proposed. 


\section{Chapter 2}

\section{Delta-Sigma ADCs}

As a starting point for discussion, this chapter presents the key operating principles of $\Delta \Sigma$ ADCs. The design parameters which can be adjusted to increase the output resolution are presented, as well as the practical limits of their application.

\subsection{Introduction to Delta-Sigma ADCs}

This is not meant to be a full tutorial on $\Delta \Sigma$ ADCs, but a basic summary of key concepts to provide a common basis for further discussion. As presented in [1], a $\Delta \Sigma \mathrm{ADC}$ achieves high resolution by trading resolution in time for resolution in amplitude. The internal circuitry of the ADC is clocked at some multiple of the required external data rate (the oversampling ratio), providing multiple internal data points that can be digitally processed to provide an output of much higher resolution than could be otherwise obtained. The effective bandwidth is limited, however, to a fraction of the achievable internal frequency.

Three of the key design parameters that affect the resolution of a $\Delta \Sigma$ ADC are: The oversampling ratio (OSR), the modulator order ( $L$ in the following equations), and the number of bits of internal quantization $(N)$. Each of these will be discussed in more depth in Section 2.2,

Figure 2.1 shows how a $\Delta \Sigma$ ADC works in comparison with a "standard" (nonoversampling or "Nyquist") ADC architecture. Simple one-bit ADCs are used for the comparison, meaning that each ADC output is quantized to a single bit. The first part of the picture shows the operation of a Nyquist ADC. The dashed line is the input to the ADC, and the circles represent ADC outputs. The one-bit ADC is very inaccurate in its representation of the input signal, since there are only two possible levels for the outputs: low and high. 

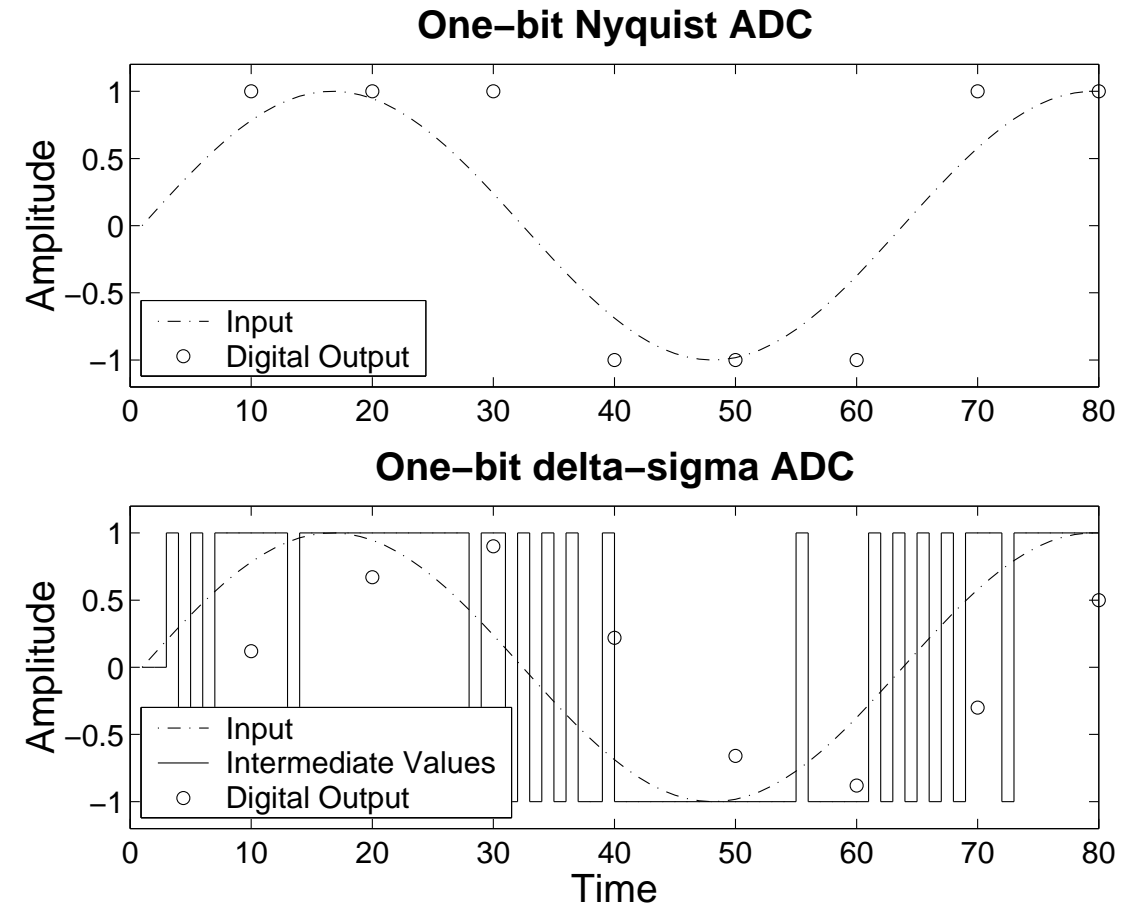

Figure 2.1: Comparison Between a Nyquist One-Bit ADC and a One-Bit $\Delta \Sigma$ ADC

The lower portion of the figure shows the operation of a one-bit $\Delta \Sigma$ converter. The input signal is the same, but the $\Delta \Sigma$ device generates ten intermediate values for each output. These intermediate values are filtered to give more precise outputs. From the plot we can see seven distinct output levels, suggesting that the one-bit ADC could have an output that has at least three bits of accuracy.

Figure 2.2 shows the circuit-level block diagram of a basic, first-order $\Delta \Sigma \mathrm{ADC}$, and Figure 2.3 shows its equivalent $\mathrm{z}$-domain model. From these it can be shown that:

$$
Y(z)=z^{-1} X(z)+\left(1-z^{-1}\right) Q(z) .
$$

It can also be shown that the noise transfer function from $Q(z)$ to the output is:

$$
\frac{Y(z)}{Q(z)}=\left(1-z^{-1}\right) .
$$

Given that $q(n)$ (the time domain representation of the signal $Q(z)$ ) is the quantization noise inserted by the internal quantizer, it is limited in magnitude to one-half an LSB 


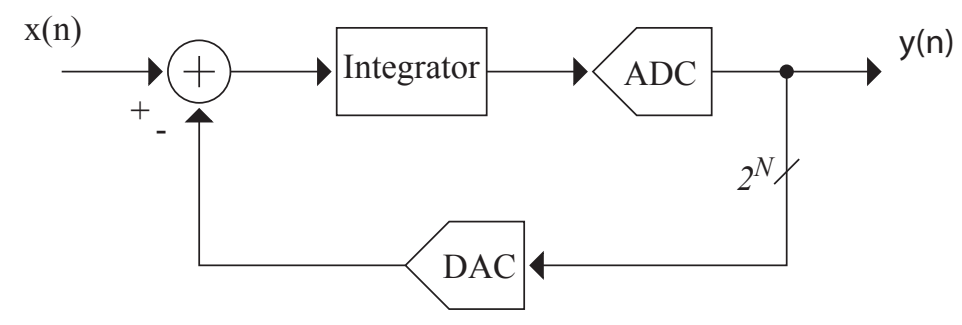

Figure 2.2: Circuit Level Block Diagram of a First-Order One-Bit $\Delta \Sigma$ ADC

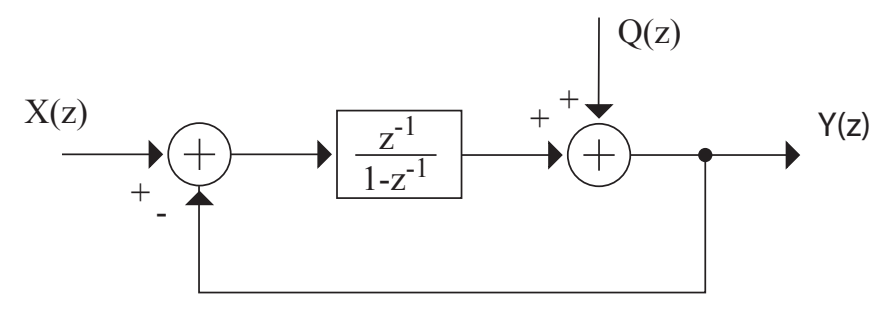

Figure 2.3: Z-Domain Model of a First-Order One-Bit $\Delta \Sigma \mathrm{ADC}$

step size (the range $\left.\left[-\frac{\Delta}{2}, \frac{\Delta}{2}\right]\right)$. In the case of one-bit quantization where the output is set to $\pm V_{\text {ref }}, \Delta$ is given by the following equation:

$$
\Delta=\frac{2 \cdot V_{\text {ref }}}{2}=V_{\text {ref }}
$$

If the input signal is sufficiently random, $q(n)$ can be assumed to be uniformly distributed within $\left[-\frac{\Delta}{2}, \frac{\Delta}{2}\right]$. Following this assumption, the probability density function (pdf) of $q(n)$ can be described as follows:

$$
P_{q}(u)=\left\{\begin{array}{ll}
\frac{1}{\Delta} & -\frac{\Delta}{2} \leq u \leq \frac{\Delta}{2} \\
0 & \text { elsewhere }
\end{array} .\right.
$$

The pdf of $q(n)$ can be shown to be zero-mean by the following method:

$$
\begin{aligned}
\mu=E\{Q\} & =\int_{-\infty}^{\infty} u \cdot P_{q}(u) \mathrm{d} u \\
& =\int_{-\frac{\Delta}{2}}^{\frac{\Delta}{2}} u \cdot \frac{1}{\Delta} \mathrm{d} u \\
& =\frac{1}{\Delta}\left[\frac{u^{2}}{2}\right]_{-\frac{\Delta}{2}}^{\frac{\Delta}{2}} \\
& =0 .
\end{aligned}
$$


Following a similar process, the variance of $q(n)$ can be shown to be a constant:

$$
\begin{aligned}
\sigma^{2}=E\left\{Q^{2}\right\} & =\int_{-\infty}^{\infty} u^{2} \cdot P_{q}(u) \mathrm{d} u \\
& =\int_{-\frac{\Delta}{2}}^{\frac{\Delta}{2}} u^{2} \cdot \frac{1}{\Delta} \mathrm{d} u \\
& =\frac{1}{\Delta}\left[\frac{u^{3}}{3}\right]_{-\frac{\Delta}{2}}^{\frac{\Delta}{2}} \\
& =\frac{\Delta^{2}}{12} .
\end{aligned}
$$

The quantization noise sequence is usually modeled as a "wide-sense stationary" (WSS) random process [7]. The power spectral density (PSD) of a WSS random process is defined as the discrete-time Fourier transform (DTFT) of the autocorrelation function. Given that the signal is zero-mean, this is equal to the covariance:

$$
S_{Q}\left(e^{j \omega}\right)=\sigma^{2} .
$$

Notice that the power is not a function of frequency; it is equal at all frequencies. This PSD characterizes the quantization noise added to the samples by the $\Delta \Sigma \mathrm{ADC}$, and the noise defines the accuracy, or SNR (signal-to-noise ratio), of the internal quantizer.

However, in the case of a $\Delta \Sigma \mathrm{ADC}$, the quantization error is not directly transmitted to the output, but is shaped by the $\left(1-z^{-1}\right)$ term in Equation 2.2 . The modulation noise, or the quantization noise's effect on the output, can be shown to be:

$$
N(z)=\left(1-z^{-1}\right) Q(z)
$$

The PSD of the shaped noise is then:

$$
\begin{aligned}
S_{N}\left(e^{j \omega}\right) & =\left|1-e^{j \omega}\right| \sigma^{2} \\
& =\left(1-e^{j \omega}\right)\left(1+e^{j \omega}\right) \sigma^{2} \\
& =[2-2 \cos (\omega)] \sigma^{2} .
\end{aligned}
$$

The modulation noise power is a function of frequency, as shown by Equation (2.9). A $\Delta \Sigma \mathrm{ADC}$ is only interested in a small portion of the signal band, so the modulation noise power in the band of interest is given by:

$$
S_{\Delta \Sigma}\left(e^{j \omega}\right)=\frac{1}{2 \pi} \int_{-\omega_{0}}^{\omega_{0}} S_{N}\left(e^{j \omega}\right) \mathrm{d} \omega
$$




$$
\begin{aligned}
& =\frac{\sigma^{2}}{2 \pi} \int_{-\omega_{0}}^{\omega_{0}}[2-2 \cos (\omega)] \mathrm{d} \omega \\
& =\frac{2 \sigma^{2}}{\pi}\left[\omega_{0}-\sin \left(\omega_{0}\right)\right] \\
& \approx \frac{2 \sigma^{2}}{\pi}\left[\omega_{0}-\omega_{0}+\frac{\omega^{3}}{6}\right] \\
& \approx \frac{\sigma^{2} \omega^{3}}{3 \pi}
\end{aligned}
$$

Given that $\omega_{0}=\omega_{s} /(2 \cdot$ OSR $)$ and that $\omega_{s}=2 \pi$, Equation 2.10 can be rewritten as follows:

$$
\begin{aligned}
S_{\Delta \Sigma}\left(e^{j \omega}\right) & \approx \sigma^{2} \cdot \frac{1}{3 \pi} \cdot \omega_{0}^{3} \\
& \approx\left(\frac{V_{\mathrm{ref}}}{\sqrt{12}}\right)^{2} \cdot \frac{1}{3 \pi} \cdot \frac{\pi^{3}}{\mathrm{OSR}^{3}} \\
& \approx\left(\frac{V_{\mathrm{ref}}}{\sqrt{12}}\right)^{2} \cdot \frac{\pi^{2}}{3} \cdot \frac{1}{\mathrm{OSR}^{3}}
\end{aligned}
$$

Figure 2.4 is a graphical comparison between the signals $Q(\omega)$ and $N(\omega)$ for a $\Delta \Sigma$ ADC with an oversampling ratio (OSR) of 16. Notice how little power of $N(\omega)$ exists in the signal band, which is all that will remain after low-pass filtering.

\subsection{Obtaining High Resolution}

The resolution of a $\Delta \Sigma$ ADC is measured by its signal-to-noise ratio (SNR). As shown in Equation 2.12, SNR is calculated by taking the root-mean-square (RMS) signal power, $S$, and dividing it by the RMS noise power in the band of interest:

$$
\mathrm{SNR}_{\text {ideal }}=10 \cdot \log _{10}\left(\frac{S}{n_{0}}\right)
$$

In order to maximize the SNR, and thus the possible resolution, of the ADC, the noise power, $n_{0}$, should be minimized.

The basic modulator of Figure 2.2 can be modified in several ways to increase the resolution. The first would be to simply run the internal clock faster relative to the output clock rate (in other words, increase the oversampling ratio). A second way is to increase the aggressiveness of the noise shaping by adding additional integration stages (this increases 


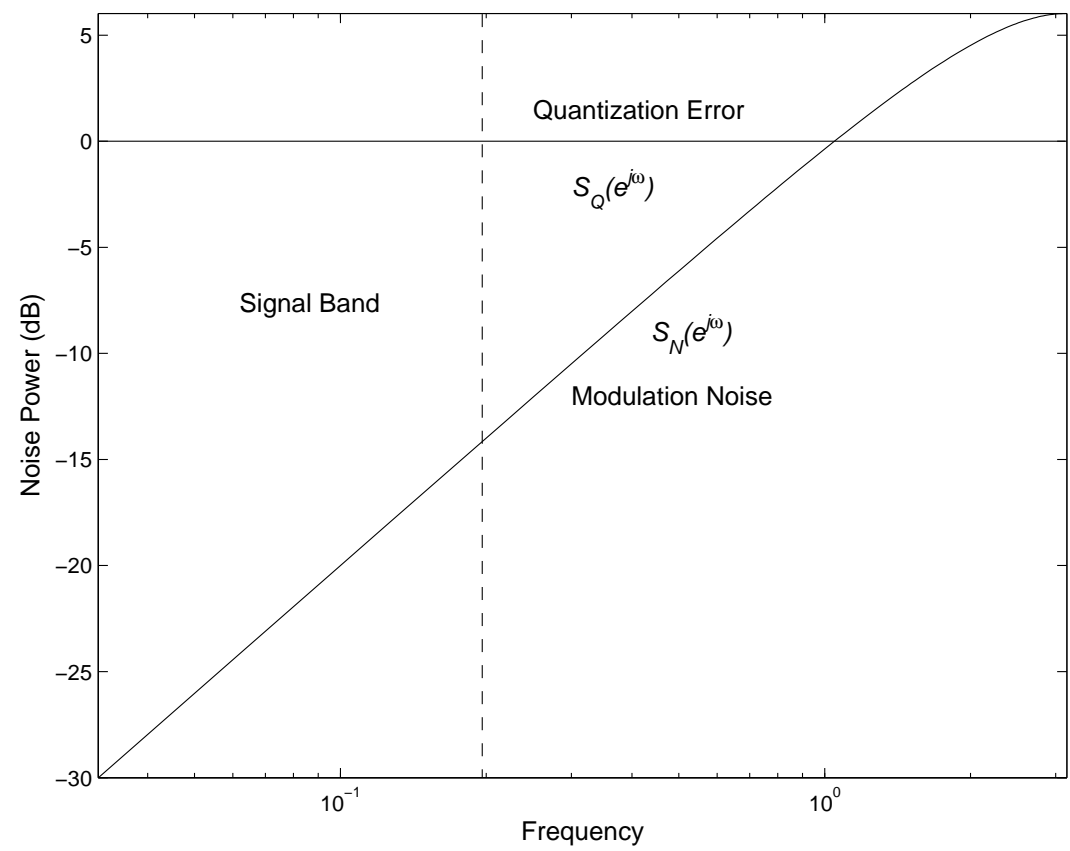

Figure 2.4: Frequency Plot Showing $S_{Q}\left(e^{j w}\right)$ and $S_{N}\left(e^{j w}\right)$ for a First-Order $\Delta \Sigma$ ADC With an OSR of 16.

the modulator order). A third method is to increase the number of bits used in the feedback path, $N$.

Equation (2.11) can be generalized to give an approximation of the ideal quantization noise in a $\Delta \Sigma \mathrm{ADC}$ :

$$
n_{0} \approx \frac{2 \cdot V_{\mathrm{ref}}}{2^{N} \sqrt{12}} \cdot \frac{\pi^{L}}{\sqrt{2 L+1}} \cdot \frac{1}{\operatorname{OSR}^{(L+0.5)}} .
$$

(It should be noted that this derivation assumes that the $\Delta \Sigma \mathrm{ADC}$ is using $\left(1-z^{-1}\right)^{L}$ noise shaping. Other noise transfer functions are possible.) This equation is comprised of the three aforementioned parameters: oversampling ratio (OSR); modulator order, $L$; and the number of bits of internal quantization, $N$. As each is increased, the ideal resolution of the ADC is increased because the overall noise signal is reduced. However, an increase in any of the three can also adversely affect power dissipation, design complexity, area required, and system stability. 
The OSR is the ratio of how fast the internal digital circuitry must run (sampling frequency, $\left.f_{s}\right)$ compared to the Nyquist rate $\left(2 f_{o}\right)$ :

$$
\mathrm{OSR}=\frac{f_{s}}{2 f_{o}}=\frac{1}{2 f_{o} T}
$$

The higher the OSR, the more aggressive the digital filtering (lower cutoff frequency) that can be done to remove more of the noise, and thus the higher the potential output resolution. However, increasing the OSR can only be done by either increasing the internal modulator frequency (which is limited by realizable device speeds), or decreasing the output bandwidth. For designs requiring high bandwidths, the OSR is limited to relatively small numbers.

The modulator order is generally the number of integration stages in the analog forward path of the $\Delta \Sigma$ ADC. Increasing the modulator order will increase the ADC resolution, but at the cost of more circuitry. This leads to a more complex design that requires more power and more chip area. Higher-order modulators are also more difficult to make stable, which sacrifices some of the aggressiveness of the noise shaping.

The internal quantization level is the number of bits of resolution the internal quantizer uses. The previous discussions in Section 2.1 used one-bit internal quantization. More internal quantization levels provide an increased resolution to the internal data points, resulting in higher overall resolution. It can also improve the overall system stability. However, this causes the internal quantizer to become more complex, increasing the chip area and required power. Perhaps more importantly, any quantization above one bit requires a DAC which must be as accurate as the overall ADC, as discussed in Section 2.3. For this reason, traditional $\Delta \Sigma$ modulators have used strictly two-level (one-bit) internal quantization.

\subsection{Multi-Bit Internal Quantization}

The problem with internal quantization greater than one bit is caused by the digitalto-analog conversion required in the $\Delta \Sigma$ modulator feedback path. As shown in Figure 2.5, the ADC output code passes through a DAC and then is summed into the analog forward path of the modulator. Any errors introduced by this DAC are added directly to the input signal and are then transmitted directly to the output along with the input. Because of this, the feedback DAC must have a resolution equal to the overall required ADC resolution. This 


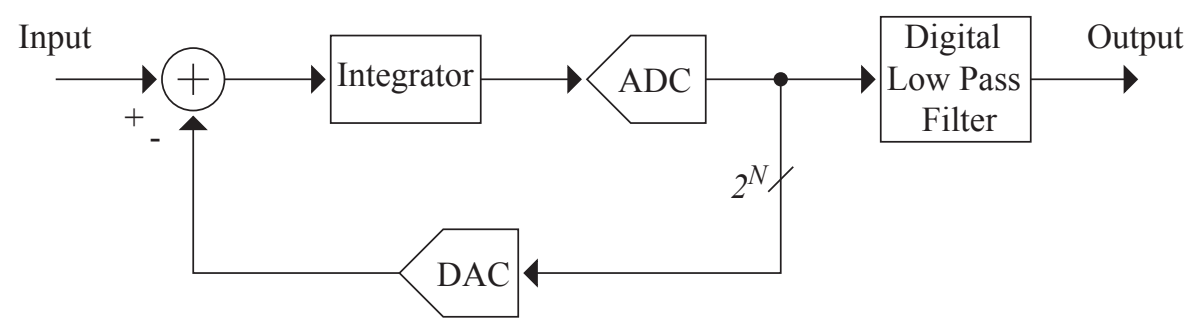

Figure 2.5: Basic First-Order $\Delta \Sigma$ ADC Block Diagram

is easily done for one-bit DACs, which have inherently perfect linearity. However, multi-bit DACs have various internal element mismatches which prevent them from realizing such high resolutions given achievable matching in typical VLSI fabrication processes.

This problem is overcome by various noise-shaping algorithms generally known as "dynamic element matching" (DEM) techniques. DEM algorithms operate on the signal at the input to the DAC, and take advantage of the oversampling inherent in $\Delta \Sigma$ devices and attempt to shape the noise generated by the DAC, shifting the noise to frequencies that are out of the band of interest. The low-pass filter present at the output of the $\Delta \Sigma$ modulator will then remove this error. DEM noise shaping is similar to $\Delta \Sigma$ noise shaping. However, DEM is done digitally where the $\Delta \Sigma$ noise shaping discussed earlier is analog, and DEM operates on noise generated from DAC element mismatches, while $\Delta \Sigma$ noise shaping operates on the quantization noise.

To understand DEM, the various DAC architectures must be described. Table 2.1 shows the three main categories of interest for this discussion. "Binary-weighted" DACs have one element for each of the input bits. A 4-bit DAC would have exactly four elements, each weighted according to the binary place value of each bit: 1:2:4:8. A "unit-element" or "thermometer-coded" DAC has as many elements as it has input codes (minus one): a 4-bit unit-element DAC would have $2^{4}-1=15$ elements, all weighted equally. "Segmented" DACs are a split between binary-weighted and unit-element DACs. A 4-bit segmented DAC may have the two least significant bits realized by $2^{2}-1=3$ elements, of weight " 1 ", and the two most significant bits realized by 3 elements of weight "4". Table 2.2 shows how various input codes would be realized by a 4-bit DAC from each category. 
Table 2.1: Different Types of DACs

\begin{tabular}{|c|c|c|}
\hline $\begin{array}{c}\text { Thermometer-Coded or } \\
\text { Unit-Element DAC }\end{array}$ & Segmented DAC & Binary-Weighted DAC \\
\hline \hline & $\begin{array}{c}2 \text { Groups: } \\
2^{N}-1 \text { elements }\end{array}$ & $\begin{array}{c}2^{k}-1 \text { MSB elements } \\
2^{l}-1 \text { LSB elements } \\
k+l=N\end{array}$ \\
& $N$ elements \\
\hline all elements & weighting of MSB to LSB & binary weighting \\
identically weighted & $2^{N-k}: 1$ & $2^{N-1}: \cdots: 2^{1}: 2^{0}$ \\
\hline
\end{tabular}

Table 2.2: Example Operation of Different Types of DACs

\begin{tabular}{|c|c|c|c|c|}
\hline Input Code & Unit-Element DAC & 2-2 Segm & ed DAC & Binary-Weighted DAC \\
\hline \multirow{3}{*}{3} & & \multicolumn{2}{|l|}{\begin{tabular}{|l|l|l|}
1 & 1 & 1 \\
\end{tabular}} & \begin{tabular}{|l|l} 
\\
\end{tabular} \\
\hline & \begin{tabular}{|l|l|l|l|l|l|l|l|} 
& 1 & 1 & 1 & 1 & 1 & 1 & 1 \\
1 & 1 & 1 & 1 & 1 & 1 & 1 \\
\end{tabular} & 4 & 4 & 2 \\
\hline & & & & 8 \\
\hline \multirow{3}{*}{5} & \multirow{3}{*}{ 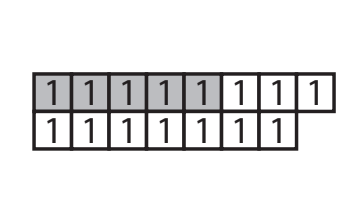 } & \multicolumn{2}{|l|}{\begin{tabular}{|l|l|l|}
1 & 1 & 1 \\
\end{tabular}} & \multirow{3}{*}{$\begin{array}{l}1 \\
2 \\
-4 \\
\end{array}$} \\
\hline & & 4 & 4 & \\
\hline & & & & \\
\hline
\end{tabular}




\begin{tabular}{|c|c|c|}
\hline Input Code $=2$ & Input Code $=3$ & Input Code $=5$ \\
\hline \begin{tabular}{|l|l|l|l|l|l|l|l|}
1 & 1 & 1 & 1 & 1 & 1 & 1 & 1 \\
\end{tabular} & \begin{tabular}{l|l|l}
1 & 1 & 1 \\
\end{tabular} & \begin{tabular}{|l|l|l|l|l|l|l|}
1 & 1 & 1 & 1 & 1 & 1 & 1 \\
\end{tabular} \\
\hline \begin{tabular}{|l|l|l|l|l|l|l|}
1 & 1 & 1 & 1 & 1 & 1 & 1 \\
\end{tabular} & \begin{tabular}{|l|l|l|l|l|l|l|}
1 & 1 & 1 & 1 & 1 & 1 & 1 \\
\end{tabular} & \begin{tabular}{|l|l|l|l|l|l|l|}
1 & 1 & 1 & 1 & 1 & 1 & 1 \\
\end{tabular} \\
\hline
\end{tabular}

Figure 2.6: Graphical Explanation of DEM Operation

DEM algorithms generally use unit-element DACs. Each element of the DAC is designed to be exactly the same size, but there is always some mismatch present. Standard unit-element DACs have a fixed error for a given input code because the same unit elements are used each time to form that code. A code of "1" will be formed with the first unit element, while a code of "4" would use the first four unit elements. DEM changes the element selection process, using different unit elements to form the same code in order to create a time-varying error.

There are several different DEM algorithms, varying mostly in how they choose which unit elements to use. The method chosen for this research is a data-weighted averaging (DWA) method, also referred to as barrel-shifting. This method keeps track of the last element used in the previous code, and uses the next group of elements sequentially. Figure 2.6 demonstrates how this works. For a code of "2" followed by a code of "3" and a code of " 5 ", the algorithm would use the first two unit elements in the DAC for the first code, followed by elements three through five for the second code. The subsequent code would then begin at the sixth element. This selection method turns each element on and off rapidly. The end result is that the DAC error is first-order noise shaped with most of the noise power at higher frequencies, as shown in [8, 9].

\subsection{High Internal Quantization}

While such DEM algorithms work well for relatively low quantization levels (two to five bits), they begin to present significant problems when internal quantization levels are extended farther. Each additional bit of internal quantization causes an exponential increase in the complexity, size, and power dissipation of the DEM logic and DAC. This is because DEM algorithms work with unit-element DACs. The DAC must have $2^{N}-1$ elements (where 
$N$ is the number of bits of internal quantization), and the DEM logic must deal with the control signals feeding those $2^{N}-1$ unit elements.

Increasing the internal quantization level also increases the size, complexity, and power usage of the internal quantizer. Fortunately, there are some architectures available that will reduce these effects, two of which are folding and two-step ADC architectures. These both generate the digital word in two parts, a "coarse" resolution and a "fine" resolution, allowing the internal quantizer to be smaller and use less power. The drawback of these architectures is that the time required to perform the quantization increases, potentially destabilizing the $\Delta \Sigma$ modulator. A folding $\mathrm{ADC}$, with its lower latency, would be the first obvious choice. Recent research has also shown that it is possible to incorporate two-step ADCs within a single-loop modulator, potentially maintaining loop stability [10, 11].

Efficient DEM algorithms are needed to accommodate the high level of quantization achieved with folding, two-step, or other coarse/fine quantizers. Research has shown that DEM algorithms based on a tree structure can be adapted for use with a segmented DAC structure [12]. An approach was desired, however, that utilizes the DWA (barrel-shifting) DEM method in search of a potentially simpler solution.

\subsection{Conclusion}

The tradeoffs involved in achieving high resolution in $\Delta \Sigma$ ADCs usually lead to low internal quantization levels and high oversampling rates. In order to achieve higher bandwidths, the OSR needs to be lowered and the internal quantization level increased. New DEM algorithms are needed to enable internal quantization levels over 5 or 6 bits while keeping chip size and power dissipation within reasonable bounds. 


\section{Chapter 3}

\section{Segmentation}

Technological advancement is always calling for faster, higher resolution ADCs. In order to have both high bandwidth and high resolution in $\Delta \Sigma$ ADCs, an efficient DEM algorithm is required to insure the accuracy of the ADC feedback path. This chapter presents one potential method of achieving such an efficient DEM application.

\subsection{Segmenting the Digital Word}

As mentioned in Section 2.4, a folding or two-step architecture for the internal quantizer can solve some of the problems arising from increasing the internal quantization level beyond 5 bits. Since these architectures provide the digital data in two sections, "coarse bits" and "fine bits", a logical way to interface with the DEM is to simply perform DEM independently on the coarse and fine DAC banks, as illustrated in Figure 3.1. With this method there is no need to encode and recombine the coarse and fine thermometer-coded signals generated by the quantizer, resulting in simple and fast feedback path circuitry. (Thermometer code is a method of encoding $N$ binary bits using $2^{N}$ signal lines. To create an output code of $k$, the first $k$ lines are set high and the rest are set low, similar to mercury in a thermometer.) The quantizer produces $N_{C}$ bits as the coarse signal and $N_{F}$ bits as the fine signal, for a total of $N$ bits $\left(N=N_{C}+N_{F}\right)$.

Figure 3.2 shows a mathematical representation of the segmented architecture from Figure 3.1. The two-step quantizer resolves the $N_{C}$ coarse bits, and then subtracts this value from the input and generates the $N_{F}$ fine bits from this signal. The gain of $2^{-\left(N-N_{C}\right)}$ inside the quantizer represents a binary right shift to insure the correct place value of the bits, since the coarse bits are the $N_{C}$ most significant bits of an $N$-bit signal. 


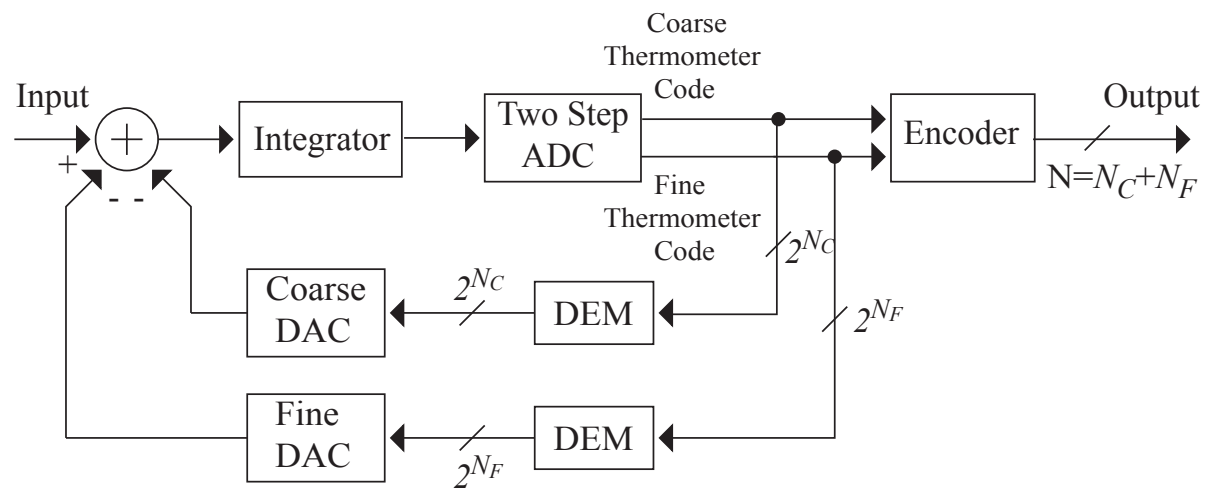

Figure 3.1: Block Diagram of a $\Delta \Sigma$ ADC With a Segmented DAC/DEM Structure

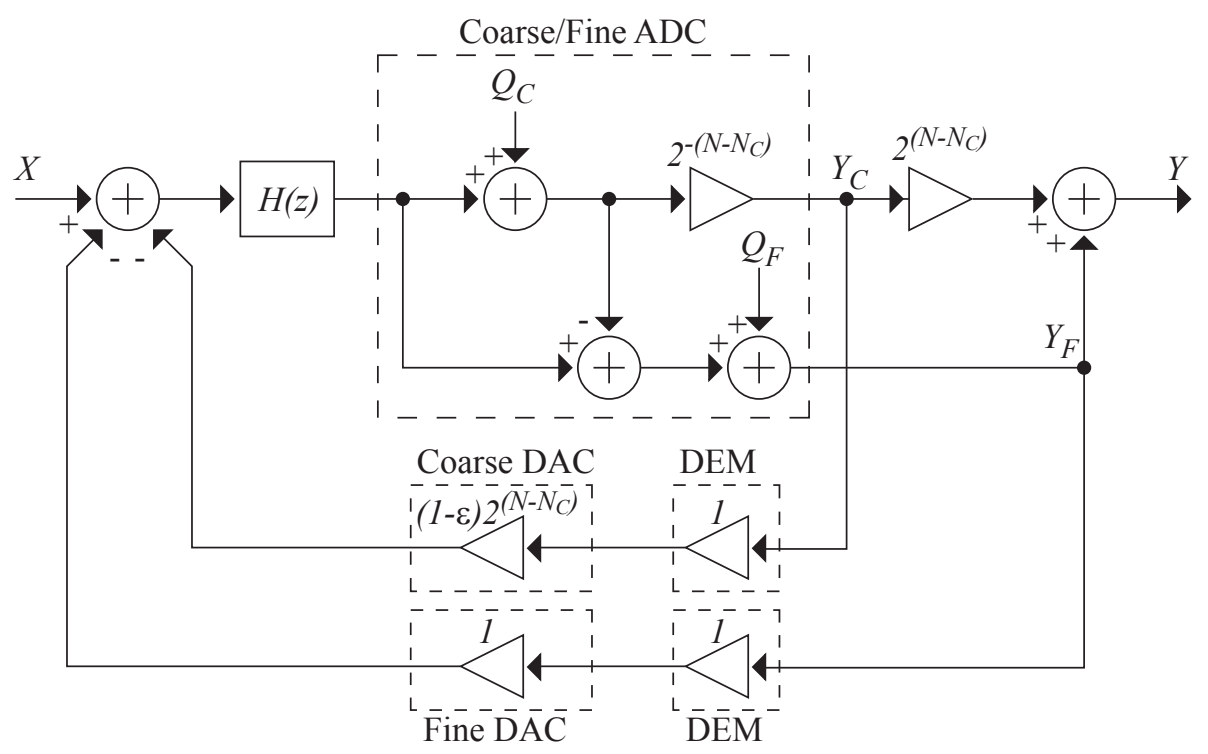

Figure 3.2: Mathematical Block Diagram of a $\Delta \Sigma$ ADC With a Segmented DAC/DEM Structure 


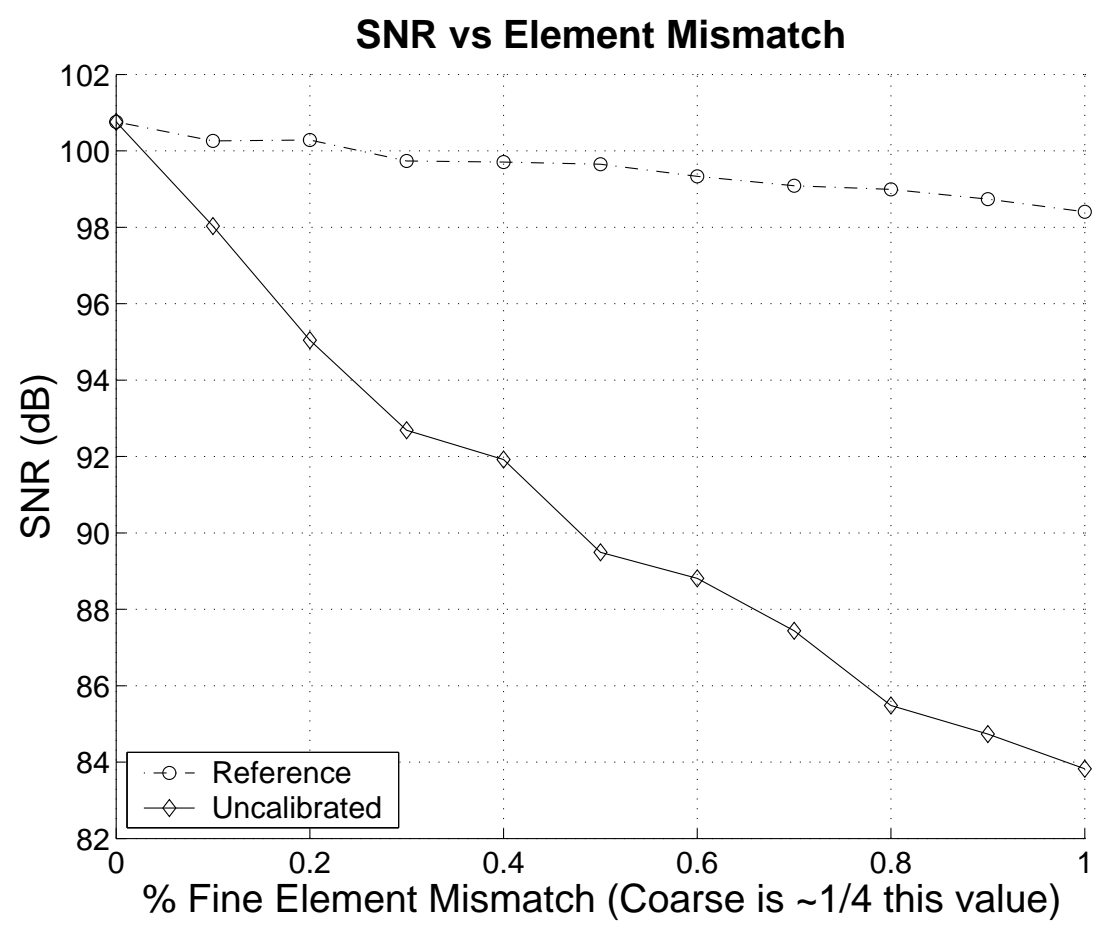

Figure 3.3: Simulation Results For a Second-Order $\Delta \Sigma$ ADC With a Segmented Feedback Path

The coarse and fine outputs are each applied to separate DACs using smaller, independent DEM circuits, significantly reducing DEM complexity. Since DEM does not change the digital signal, but only operates on the error within each DAC, the DEM blocks can be represented as a simple gain of unity, as seen in Figure 3.2. The coarse DAC transfer function is weighted $2^{N-N_{C}}$ times that of the fine DAC to represent the place value of the coarse bits relative to the fine. In practice, this is done by making the elements of the coarse DAC $2^{N-N_{C}}$ times larger than those of the fine DAC. The quantization noise, $Q_{C}$, present in both signals $Y_{C}$ and $Y_{F}$, ideally will cancel when the coarse and fine signals are summed together at the modulator input. This result should be the same as if a single DEM circuit with a single DAC had been in the feedback path. With perfect DACs (0\% mismatch), MATLAB behavioral simulations indicate that this system operates well, as shown in Figure 3.3. But, as shown in the same Figure, with the addition of unit-element mismatch, the overall SNR of the system drops much faster than the full 256-element reference case. 
Unless otherwise noted, all simulations performed for comparison in this thesis use a second-order $\Delta \Sigma$ ADC architecture with an OSR of 20 and 8 bits of internal quantization. The reference case is a non-segmented (single-path) architecture, and the others use some variation of the segmented architecture, splitting the feedback signal into 4 bits for each of the coarse and fine paths. The percent mismatch of the coarse unit elements is scaled assuming they are $2^{N-N_{C}}$ times larger than the fine unit elements, which is typically the case in a practical application. Random element sizes are generated using the MATLAB "rand" function. To account for the randomness of the element mismatch, the average of 21 different simulations is taken, with new element values generated for each run.

\subsection{Analysis of the Problem}

The previous explanation of the system's operation neglected an important point. The weighting of the coarse DAC as compared to the fine DAC depends on the relative sizes of the unit elements. Since the unit elements vary in actual size, the weight of the coarse DAC will not be exactly $2^{N-N_{C}}$, but will be off by the factor $1-\epsilon$, as shown in Figure 3.2 . Because of this gain mismatch between the coarse and fine DAC banks, the quantization noise, $Q_{C}$, will not completely cancel when the coarse and fine signals are summed together, as they would in the ideal case. The non-canceled portion of the quantization noise will be added directly to the input signal, and thus be transmitted to the output of the ADC.

The output, $Y$, of the ADC in Figure 3.2 can be derived as follows:

$$
\begin{aligned}
& Y_{C}(z)=\frac{2^{-\left(N-N_{C}\right)}\left[Q_{C}(z)+\left(X(z)-Y_{F}(z)\right) H(z)\right]}{1+(1-\epsilon) H(z)} \\
& Y_{F}(z)=\frac{\left(-Q_{C}(z)+Q_{F}(z)\right)[1+(1-\epsilon) H(z)]}{1+(1-\epsilon) H(z)} \\
& Y(z)=Y_{C}(z) \cdot 2^{N-N_{C}}+Y_{F}(z) \\
& =\underbrace{\frac{X(z) \cdot H(z)}{1+(1-\epsilon) H(z)}}_{\text {Desired Signal }}+\underbrace{\frac{Q_{F}(z)}{1+(1-\epsilon) H(z)}}_{\text {Expected Noise }} \\
& +\underbrace{\frac{\epsilon \cdot\left(Q_{C}(z)-Q_{F}(z)\right) H(z)}{1+(1-\epsilon) H(z)}}_{\text {Extra Noise Term }} .
\end{aligned}
$$


For comparison, a non-segmented (single-path) approach would lead to:

$$
Y(z)=\underbrace{\frac{X(z) \cdot H(z)}{1+H(z)}}_{\text {Desired Signal }}+\underbrace{\frac{Q(z)}{1+H(z)}}_{\text {Expected Noise }}
$$

A comparison of Equations (3.4) and 3.5 shows that the coarse/fine system has the error term " $\epsilon \cdot\left(Q_{C}-Q_{F}\right)$ " present in addition to normal quantization noise. The values of $Q_{C}$ and $Q_{F}$ are dependent on the number of bits used in the coarse and fine signals $\left(N_{C}\right.$ and $N_{F}$ respectively) and the current input signal. In normal implementations, $Q_{C}>>Q_{F}$ because the LSB step size of the coarse bits is much larger than the LSB step size of the fine bits, so $Q_{C}-Q_{F} \approx Q_{C}$. The value of the mismatch term, $\epsilon$, changes with each clock cycle due to the operation of the DEM. Its magnitude depends on the mismatch between coarse and fine DACs, which is a function of mismatch between unit elements. For realistic unit-element mismatch values, $\epsilon$ is small, but still large enough to significantly affect the SNR of the system. Simulations show that a segmented $\Delta \Sigma$ ADC, as described in this section, with a unit-element mismatch of $1.0 \%$ has an SNR of $84 \mathrm{~dB}$ as compared to almost $99 \mathrm{~dB}$ for the reference case (see Figure 3.3.

\subsection{Conclusion}

Handling the coarse and fine data separately is an appealing option. It requires very little hardware, can be very fast, and is simple and intuitive. However, simulations and mathematical analysis show that this method has some serious problems and will degrade the overall SNR of the system. The problem lies in the mismatch between the coarse and fine DACs. Because the unit elements in each DAC will each have an element of random error in their size, the ratio of the coarse DAC weighting to that of the fine will not be the desired value, and will even vary with time due to the DEM circuitry's operation. This results in coarse quantization noise leaking to the output, which degrades SNR substantially. 


\section{Chapter 4}

\section{Calibration}

Using a segmented feedback DAC with separate DEM is a potential method to simplify the DEM block and allow it to operate on highly-quantized signals. However, error is introduced by another mismatch problem, namely, the mismatch between the coarse and fine DAC banks. As the title suggests, this chapter presents a method to remove that mismatch using calibration.

\subsection{The Motivation For Calibration}

The mismatch term, $\epsilon$, from Equation (3.4) represents the deviation from the desired gain ratio of the coarse and fine DACs. As mentioned in the previous section, each DAC's gain changes with every cycle due to the DEM operation. However, since $\Delta \Sigma$ ADCs use oversampling, the average gain of the DAC over time is more important than any instantaneous value. For an $N$-bit, fully-differential DAC with $M=2^{N}-1$ unit elements, each of value $D_{i}$, the output corresponding to an input code of " $n$ " in the range $[0, N]$ is equal to:

$$
F_{D}(n)=\sum_{i=1}^{n} D_{i}-\sum_{i=n+1}^{M} D_{i} .
$$

In words: the output is equal to the sum of the first $n$ elements minus the sum of the remaining elements. The difference between two successive input codes is then given by:

$$
F_{D}(k)-F_{D}(k-1)=2 \cdot D_{k}
$$

Since the DAC gain is equal to the average slope of the transfer function, the average gain of the DAC $(\bar{A})$ can be written as:

$$
\bar{A}=\frac{\overline{F_{D}(k)-F_{D}(k-1)}}{2 D_{\mathrm{ref}}}=\frac{\overline{D_{i}}}{D_{\mathrm{ref}}}
$$


Table 4.1: Simulated SNR Results for Segmented $\Delta \Sigma$ ADC (in dB)

\begin{tabular}{|c|c|c|c|}
\hline \multirow{2}{*}{} & \multicolumn{3}{|c|}{ Unit-Element \% Mismatch } \\
\cline { 2 - 4 } & 0.0 & 0.5 & 1.0 \\
\hline Reference & 100.8 & 99.6 & 98.4 \\
Segmented & 100.8 & 89.5 & 83.8 \\
Segmented with & 100.8 & 99.5 & 98.0 \\
\hline Adjusted DAC Gain & & \\
\hline
\end{tabular}

where $2 D_{\text {ref }}$ represents the ideal output step size (corresponding to an input LSB change) and $\overline{D_{i}}$ is the average unit-element value.

Equation (4.3) states that the average gain of the coarse or fine DAC is equal to the normalized average element size. So the mismatch, $\epsilon$, from Equation (3.4) can be reduced by matching the average element values between DACs with the ratio of $2^{N-N_{C}}: 1$. The individual DAC element values are not important, as long as the average element values meet this ratio. The $(1-\epsilon)$ factor will be zero on average and the coarse quantization noise will not leak to the output. Simulated results shown in Table 4.1 demonstrate this. The first row is the SNR of the reference $\Delta \Sigma \mathrm{ADC}$ at various unit-element mismatch values. The second row shows the standard segmented case. The third row is the same segmented case, with a single fine unit element adjusted such that the average unit-element value in the coarse DAC is exactly 16 times larger than the average unit-element value in the fine DAC $\left(2^{8-4}: 1=16: 1\right)$. At $1 \%$ mismatch, the segmented ADC without equalized DAC gains has an SNR 14.6 dB lower than the reference (non-segmented) case. In comparison, the ADC with adjusted DAC gains has an SNR only $0.4 \mathrm{~dB}$ lower than the reference case. Such adjustment of the DAC gains can be acheived through calibration.

\subsection{The Calibration Method}

Figure 4.1 is a block diagram of a proposed calibration architecture. This method performs the calibration as an initialization step. During calibration, the connections from the coarse/fine ADC to the feedback path are broken and a single-bit path provides the 


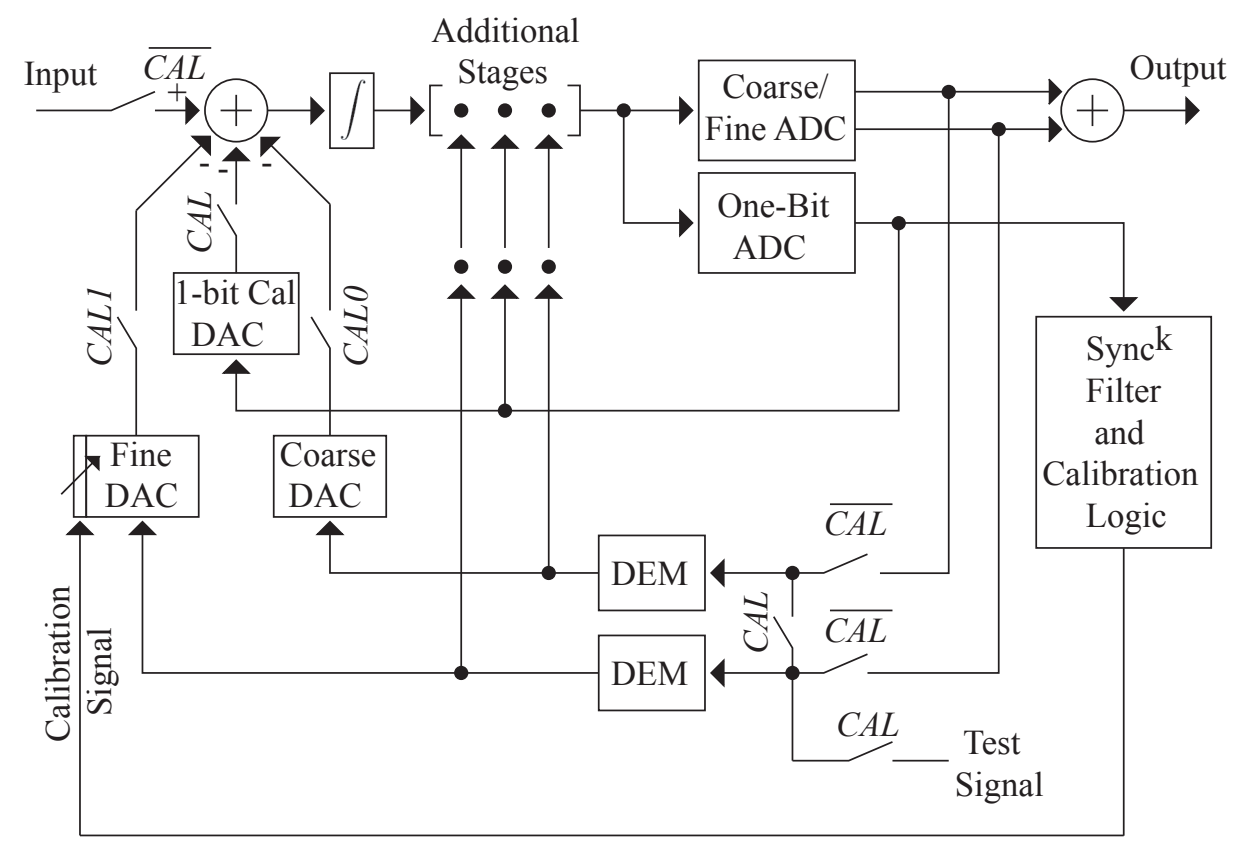

Figure 4.1: Block Diagram of Calibration Method

modulator feedback. A one-bit quantizer is used because it is immune to the mismatch that plagues multi-bit quantizers. The modulator input is grounded and a fixed, DC test signal is presented to the coarse and fine DACs through the DEM circuitry. The output is sent to an averaging sync ${ }^{k}$ filter, and then measured for each DAC individually, with the other DACs disconnected from the circuit. The relative measurement between coarse and fine DAC banks can then be used to make the necessary adjustment to match the ratio of the average DAC element values. Simulations show that the calibration routine must measure coarse and fine average capacitor values to within $2.5 \%$ of their nominal values to keep SNR penalties below $2 \mathrm{~dB}$. This means that the one-bit modulator must measure the calibration signal to within $0.0098 \%$ of full scale (13 to 14-bit accuracy); this is feasible for a one-bit modulator with high oversampling.

Correction must be applied to one of the DACs to complete the calibration. Due to the averaging effect of the DEM algorithm, the required adjustment does not have to be added to all the unit elements in the bank. Simulations show that it is sufficient to manipulate a single fine unit element to achieve the required average unit-element ratio. 
Similarly adjusting a coarse bank element is not effective; two possible explanations for this are as follows: 1) the mismatch between individual coarse bank elements may be increased, reducing the effectiveness of coarse-bank DEM, and 2) the coarse DAC input data is more strongly patterned than the fine DAC input data, therefore DEM averaging is performed less efficiently in the coarse DAC.

\subsection{Drawbacks and Benefits}

A distinct advantage of the calibration method is the low complexity of the feedback path. For an 8-bit quantizer, two independent, 4-bit DEM implementations are required. These DEM algorithms can be constructed so that only a four-stage pass-gate structure is required in the signal path to shuffle the thermometer-coded ADC outputs [13]. Thus both the complexity and the timing delay of the digital feedback path are minimal.

The necessity of having a separate calibration mode is a significant drawback. The device is not ready for immediate use, and cannot track changes in circuit behavior relating to temperature and other time-varying effects. A possible solution is to use a background calibration routine, such as one that uses an out-of-band DAC test sequence as proposed in [14]. Another drawback is that the calibration circuitry can be quite complex and large, requiring a lot of control circuitry and memory to compute and apply the required calibration adjustment. Also, as separate DACs are generally used for each integration stage in $\Delta \Sigma$ modulators, each set of DAC banks must be calibrated individually, which adds to the time required to calibrate high-order modulators. Exactly how to apply the calibration adjustment in such a way that does not add additional error is also a non-trivial problem.

\subsection{Behavioral Simulation Results}

Figure 4.2 shows simulation results for both calibrated and uncalibrated systems compared to the reference case for various unit-element mismatch percentages. In the calibration simulation, a "calibration mode" is simulated using a 1-bit ADC and feedback path, and alternately applying a test signal to the coarse and fine DAC to measure their individual effects on the output. These individual measurements are then compared and used to calculate how much a single fine element must be adjusted to insure an average element size 


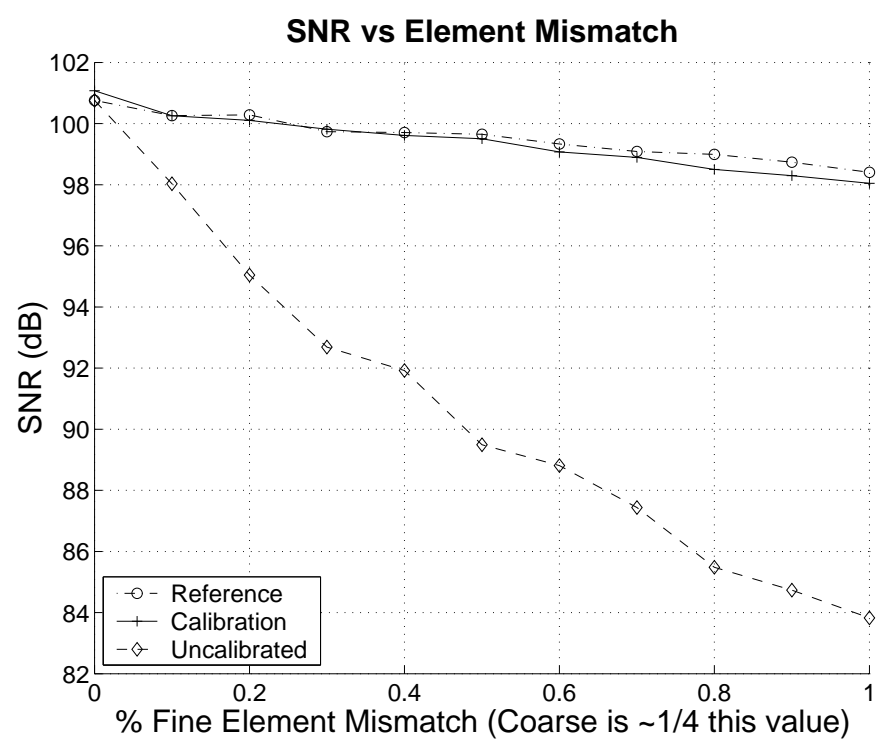

Figure 4.2: Simulated Results for Calibrated System

ratio of $16: 1$. The simulation then continues normal operation with this calibration amount in place.

For the uncalibrated system and $1 \%$ mismatch, using independent coarse/fine DEM results in a $14.5 \mathrm{~dB}$ degradation in SNR from the ideal case. For the calibrated simulation, one of the fine DAC elements was adjusted to correct the gain ratio between the coarse and fine DACs. This small adjustment achieved an SNR within $0.5 \mathrm{~dB}$ of the reference case. (Note that this does not take into account errors in applying the calibration adjustment.)

\subsection{Conclusion}

Calibration can significantly improve the SNR of the subdivided system, and can be done in a way to minimally affect the overall operation of the ADC. The method proposed adds a one-bit calibration path and extra circuitry that are active during an initialization step to perform the calibration, and a small bit of logic to vary one of the fine unit elements. It does not add extra logic to the feedback path, and does not change the normal operation of the ADC. As such, it is fairly simple, and can be quite accurate. However, it is unable to track changes in the unit-element values that can occur over time, and the calibration measurement and adjustment circuitry can be complex. Also, the calibration must be performed for 
each group of DAC banks, meaning that increasing the order of the $\Delta \Sigma$ modulator will significantly increase the time required to fully calibrate the ADC. 


\section{Chapter 5}

\section{Requantization}

The previous chapter showed that calibration is a viable option for eliminating the error added by the segmented DEM approach. However, the operation of the DEM blocks themselves suggests another solution. The DEM blocks function not by removing the error in the DAC elements, but by shifting the noise caused by that error away from the band of interest. This chapter presents a noise-shaping method that attempts to do the same with the noise caused by the coarse and fine DAC bank mismatch.

\subsection{The Noise-Shaped Requantization (ReQ) Method}

This method was initially proposed in [6] for a $\Delta \Sigma$ DAC; this work extends this concept to $\Delta \Sigma$ ADCs. The basic idea is to generate a new coarse signal with a digital $\Delta \Sigma$ modulator and use this coarse signal to generate a new fine signal. This insures that both the coarse and fine signals are individually noise shaped, which is performed in a way that causes the quantization error leakage to be noise shaped as well. Even though it does not completely cancel errors due to DAC mismatch, the quantization error noise power will be outside the signal band. The process is explained below, and is modeled in Figure 5.1.

First, the coarse and fine signals from the ADC internal quantizer are encoded into binary and concatenated to form a 2's complement $N$-bit signal, $Y^{\prime}$. This signal is then requantized to $N_{C}$ bits to form a new coarse signal using a digital first-order $\Delta \Sigma$ modulator. This coarse signal is subtracted from the original $N$-bit signal to form the new fine signal, comprised of $N_{F}+1$ bits. After requantization, the new coarse and fine signals are:

$$
Y_{C}^{\prime}(z)=2^{-\left(N-N_{C}\right)}\left[Y^{\prime}(z)+Q_{C}^{\prime}(z)\left(1-z^{-1}\right)\right]
$$




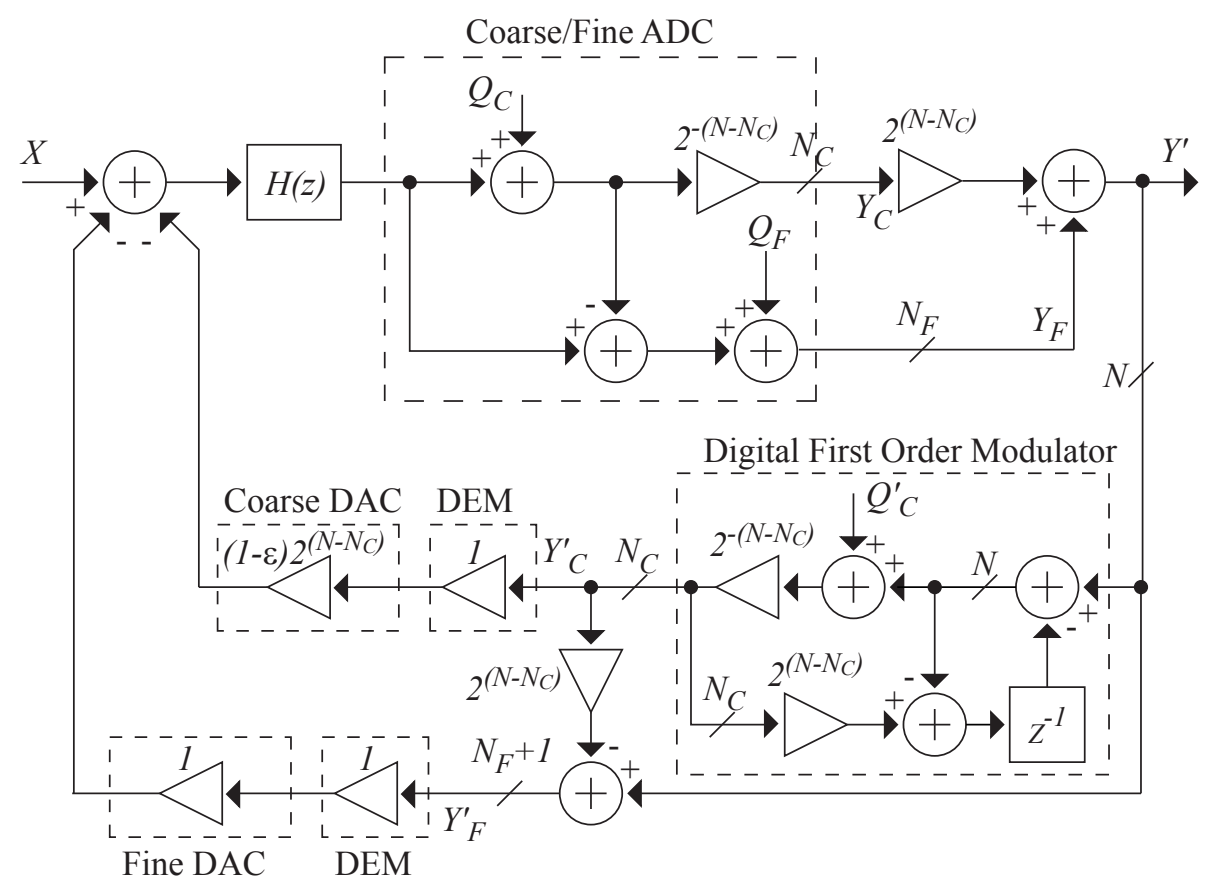

Figure 5.1: Mathematical Block Diagram of ReQ Architecture

$$
Y_{F}^{\prime}(z)=-Q_{C}^{\prime}(z) \cdot\left(1-z^{-1}\right) .
$$

Signals $Y_{C}^{\prime}(z)$ and $Y_{F}^{\prime}(z)$ then pass through independent DEM blocks and DACs and are summed at the input of the modulator. With first-order requantization (ReQ), the quantization error that is not completely cancelled due to coarse/fine DAC mismatch (as in Equation (3.4) is noise-shaped away from the signal band. The output of the system with ReQ as in Figure 5.1 is derived as follows:

$$
\begin{aligned}
Y_{C}(z) & =2^{-\left(N-N_{C}\right)}\left[Q_{C}(z)+\left(X(z)-(1-\epsilon) 2^{N-N_{C}} \cdot Y_{C}^{\prime}(z)-Y_{F}^{\prime}(z)\right) H(z)\right] \\
Y_{F}(z) & =-Q_{C}(z)+Q_{F}(z) \\
Y^{\prime}(z) & =Y_{C}(z) \cdot 2^{N-N_{C}}+Y_{F}(z) \\
& =X(z) \cdot H(z)-\left[(1-\epsilon) Q_{C}^{\prime}(z)-Q_{C}^{\prime}(z)\right]\left(1-z^{-1}\right) \\
& \quad+Q_{F}(z)-(1-\epsilon) H(z) \cdot Y^{\prime}(z) \\
& =\underbrace{\frac{X(z) \cdot H(z)}{1+(1-\epsilon) H(z)}}_{\text {Desired Signal }}+\underbrace{\frac{Q_{F}(z)}{1+(1-\epsilon) H(z)}}_{\text {Expected Noise }}
\end{aligned}
$$




\section{Power Spectra}
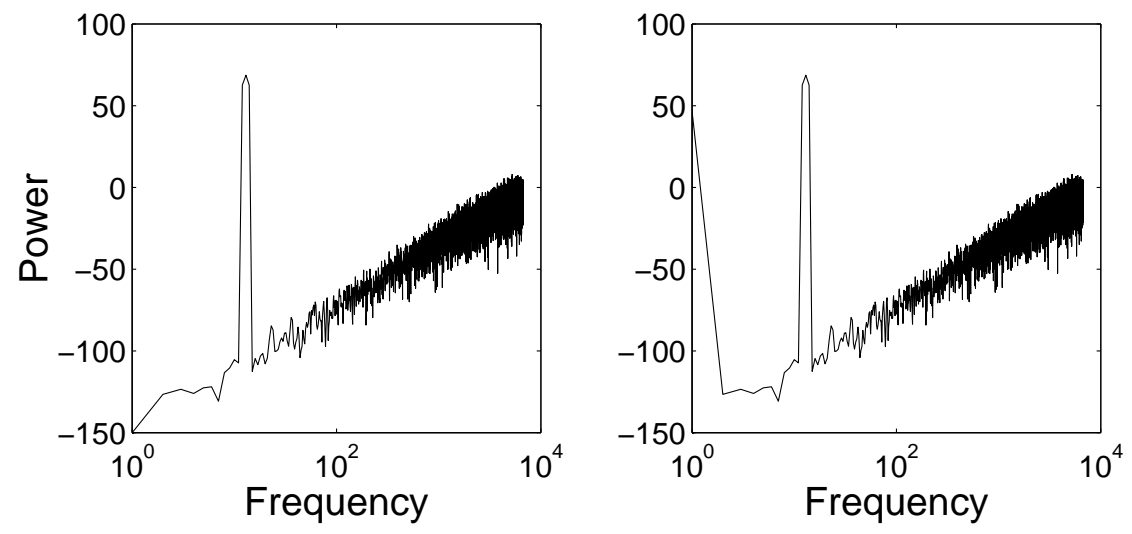

Figure 5.2: Comparison of an Ideal Segmented $\Delta \Sigma$ ADC (as in Figure 3.2) and an Ideal $\Delta \Sigma$ ADC Using the ReQ Block Showing DC Offset

$$
+\underbrace{\frac{\left(\epsilon \cdot Q_{C}^{\prime}(z)\right)\left(1-z^{-1}\right) H(z)}{1+(1-\epsilon) H(z)}}_{\text {Extra Noise Term }} .
$$

A comparison of Equation (5.3) and Equation (3.4) shows that the desired signal and expected noise terms are almost the same, but the extra noise term in Equation (5.3) is now multiplied by $\left(1-z^{-1}\right)$, which causes first-order noise shaping. Simulations show that higher-order ReQ is not necessary; first-order ReQ sufficiently suppresses coarse/fine mismatch errors below the noise shaped by the DEM within each DAC.

\subsection{Drawbacks and Benefits}

Although the need for calibration is eliminated, the ReQ method contains several minor drawbacks. An extra fine bit is required because of the final addition operation before the DEM blocks in Figure 5.1, requiring slightly larger DEM and DAC implementations. The total number of bits of information is still the same ( $N$ bits); the coarse and fine signals $Y_{C}^{\prime}$ and $Y_{F}^{\prime}$ overlap by one bit. Another potential drawback is a slight reduction in signal range due to the possibility of overflow in the ReQ circuitry. Simulations show that the SNR is not adversely affected by potential overflow if the input is limited to about $90 \%$ of full scale. 
A third drawback comes in the form of a DC error that occurs when using the ReQ method. Even when simulations are performed using ideal unit elements, some DC offset is present. Figure 5.2 demonstrates this. The left half of the picture shows the power spectrum for a segmented $\Delta \Sigma \mathrm{ADC}$ with ideal elements. The right half shows the same signal, this time from a $\Delta \Sigma$ ADC with ReQ operating in the feedback path. The ReQ signal shows first-order noise shaping of the quantization noise leakage, but it also has a DC component. This DC offset arises due to the difference between $Y_{F}$ and $Y_{F}^{\prime}$. The signal $Y_{F}$ is an unsigned number (the lower bits of a 2's complement number), while $Y_{F}^{\prime}$ is a 2's complement number, which can be either positive or negative. An analysis of the source of this DC error follows.

In a system using unsigned numbers (positive integers), a fully-differential DAC can be modeled by the following equation:

$$
D(Y)=\frac{V_{\text {ref }}}{2^{N}-1}(Y)-\frac{V_{\text {ref }}}{2^{N}-1}\left[\left(2^{N}-1\right)-(Y)\right]
$$

(A fully-differential DAC is one that always uses all of the elements, each in either the "positive" or the "negative" sense.) In a 2's complement DAC, the equation for $D(Y)$ is slightly different:

$$
D(Y)=\frac{V_{\mathrm{ref}}}{2^{N}-1}\left(Y+2^{N-1}\right)-\frac{V_{\mathrm{ref}}}{2^{N}-1}\left[\left(2^{N}-1\right)-\left(Y+2^{N-1}\right)\right] .
$$

The first term represents the elements that are "on," or added, and the second term represents the elements that are "off," or subtracted. In these equations, $V_{\text {ref }}$ is the reference voltage for the DAC, and $2^{N}-1$ is representative of how many individual levels the DAC can represent. The term $Y+2^{N-1}$ in Equation (5.5) results from the need to convert the 2 's complement number " $Y$ " to a positive number representing how many unit elements to use in the "positive" sense (the rest are "negative", as shown by the second term in the equation). Through some simplification it is easy to show that the 2's complement DAC equation reduces to:

$$
D(Y)=\frac{V_{\text {ref }}}{2^{N}-1}(2 \cdot Y+1)
$$


For a basic coarse/fine system with no ReQ step inserted, as in Figure 3.2 , since $Y_{C}$ is a 2's complement number, and $Y_{F}$ is an unsigned number, the following equations describe the output of the DACs:

$$
\begin{aligned}
D_{C}\left(Y_{C}\right)= & \frac{V_{\text {ref }} \cdot 2^{N-N_{C}}}{2^{N}-1}\left(Y_{C}+2^{N_{C}-1}\right) \\
& -\frac{V_{\text {ref }} \cdot 2^{N-N_{C}}}{2^{N}-1}\left[\left(2^{N_{C}}-1\right)-\left(Y_{C}+2^{N_{C}-1}\right)\right] \\
D_{F}\left(Y_{F}\right)= & \frac{V_{\text {ref }}}{2^{N}-1}\left(Y_{F}\right)-\frac{V_{\text {ref }}}{2^{N}-1}\left[\left(2^{N_{F}}-1\right)-\left(Y_{F}\right)\right] .
\end{aligned}
$$

The sum of the output of these two DACs with the input of $Y_{C}$ and $Y_{F}$ should be the same as the output of a single DAC with the input of $Y$ (see Equation (5.6)):

$$
\begin{aligned}
D_{C}\left(Y_{C}\right)+D_{F}\left(Y_{F}\right)= & \frac{V_{\text {ref }}}{2^{N}-1}\left[2 \cdot 2^{N-N_{C}}\left(Y_{C}+2^{N_{C}-1}\right)+2\left(Y_{F}\right)\right. \\
& \left.-2^{N-N_{C}}\left(2^{N_{C}}-1\right)-\left(2^{N_{F}}-1\right)\right] \\
= & \frac{V_{\text {ref }}}{2^{N}-1}\left[2\left(2^{N-N_{C}} \cdot Y_{C}+Y_{F}+2^{N-N_{C}} \cdot 2^{N_{C}-1}\right)\right. \\
& \left.-\left(2^{N}-1\right)-\left(-2^{N-N_{C}}+2^{N_{F}}\right)\right] \\
= & \frac{V_{\text {ref }}}{2^{N}-1}\left[2\left(Y+2^{N-1}\right)-\left(2^{N}-1\right)\right] \\
= & \frac{V_{\text {ref }}}{2^{N}-1}(2 \cdot Y+1) .
\end{aligned}
$$

This shows that the two separate DACs together perform exactly the same function that a single DAC (as in the reference design) would do: $D(Y)=D_{C}\left(Y_{C}\right)+D_{F}\left(Y_{F}\right)$.

When this same analysis is applied to the ReQ system (as in Figure 5.1), a slightly different result is obtained. Both $Y_{C}^{\prime}$ and $Y_{F}^{\prime}$ are 2's complement numbers, and $Y_{F}^{\prime}$ has one more bit than $Y_{F}$. So the DAC equations are:

$$
\begin{aligned}
D_{C}^{\prime}\left(Y_{C}^{\prime}\right)=\frac{V_{\mathrm{ref}} \cdot 2^{N-N_{C}}}{2^{N}-1} & \left(Y_{C}^{\prime}+2^{N_{C}-1}\right) \\
& \quad-\frac{V_{\mathrm{ref}} \cdot 2^{N-N_{C}}}{2^{N}-1}\left[\left(2^{N_{C}}-1\right)-\left(Y_{C}^{\prime}+2^{N_{C}-1}\right)\right] \\
D_{F}^{\prime}\left(Y_{F}^{\prime}\right)=\frac{V_{\text {ref }}}{2^{N}-1}\left(Y_{F}^{\prime}+2^{N_{F}}\right) & \\
& -\frac{V_{\mathrm{ref}}}{2^{N}-1}\left[\left(2^{N_{F}+1}-1\right)-\left(Y_{F}^{\prime}+2^{N_{F}}\right)\right] .
\end{aligned}
$$


The sum of $D_{C}^{\prime}\left(Y_{C}^{\prime}\right)$ and $D_{F}^{\prime}\left(Y_{F}^{\prime}\right)$ should be the same as a single DAC with an input of $Y^{\prime}$. However, it is not. The sum is instead given by:

$$
\begin{aligned}
D_{C}^{\prime}\left(Y_{C}^{\prime}\right)+D_{F}^{\prime}\left(Y_{F}^{\prime}\right)= & \frac{V_{\text {ref }}}{2^{N}-1}\left[2 \cdot 2^{N-N_{C}}\left(Y_{C}^{\prime}+2^{N_{C}-1}\right)+2\left(Y_{F}^{\prime}+2^{N_{F}}\right)\right. \\
= & \frac{V_{\text {ref }}}{2^{N-N_{C}}-1}\left[2\left(2^{N_{C}}-1\right)-\left(2^{N_{F}+1}-1\right)\right] \\
& \left.-\left(2^{N}-1\right)+\left(2 \cdot 2^{N_{F}}+2^{N-N_{C}}-2^{\prime}+Y_{F}^{\prime}+1\right)\right] \\
= & \frac{V_{\text {ref }}}{2^{N}-1}\left[2\left(2^{N-N_{C}} \cdot 2_{C}^{\prime}+Y_{F}^{\prime}+2^{N-1}\right)\right. \\
= & \frac{V_{\text {ref }}}{2^{N}-1}\left[2\left(Y+2^{N-1}\right)-\left(2^{N}-1\right)+\left(2^{N_{F}-1}\right)\right] \\
= & \underbrace{\frac{V_{\text {ref }}}{2^{N}-1}(2 \cdot Y+1)}_{\text {Desired Signal }}+\underbrace{\frac{V_{\text {ref }}}{2^{N}-1}\left(2^{N_{F}}\right)}_{\text {Offset }} .
\end{aligned}
$$

The offset term is added to the input node of the $\Delta \Sigma$ ADC, causing a DC offset in the output. It is caused by the fact that $Y_{F}^{\prime}$ is a signed number, whereas before, $Y_{F}$ was unsigned. (The examples given were for a 2's complement number representation. It can be shown that the same DC offset is present for other signed numbering systems, such as offset or signmagnitude notation.)

The DC offset is the same magnitude as the step caused by one coarse unit element. To counter this offset, one extra unit element was added to the coarse DAC. Given the fullydifferential nature of the system, the additional unit element will effectively be subtracted from the DAC output signal, removing the DC bias. With unit-element mismatch present, it does not fully remove the DC offset, but does so accurately enough to not noticeably affect the overall SNR of the ADC.

Since the ReQ method adds significant logic to the feedback path, it can potentially limit the maximum possible clock speed of the device. If a folding ADC quantizer is used, one-half clock cycle (less the ADC comparator latching time) is available for this digital computation. As an example, the design presented later in this work has a target clock rate of $50 \mathrm{MHz}$, permitting about $10 \mathrm{~ns}$ for the computation. Recent developments show that a 
two-step ADC architecture can be used while still allowing this same amount of propagation time for the logic [10, 11]. With careful design, the delay through this path should be small enough, and as CMOS technology scales, the delay will continue to decrease, permitting faster clock rates.

The benefits to offset these drawbacks are few, but significant. First, there is no startup mode required, providing "instant-on" functionality. Second, since this method of removing the DAC mismatch error is completely digital, the actual chip should operate very close to the simulation. In the case of calibration, there are additional inaccuracies that will be present in physical silicon (such as the circuits to apply the calibration change and a limited number of bits to represent the measurements) that were not taken into account in the simulations. So the ReQ method is potentially easier to implement and easier to achieve functionality in silicon.

\subsection{Behavioral Circuit Simulation Results}

Figure 5.3 compares the performance of the calibration and ReQ methods against the ideal (full DEM) case. For the case of $1 \%$ fine element mismatch, the ReQ method achieves an average SNR of $96.5 \mathrm{~dB}$, which is only $2 \mathrm{~dB}$ less than the full 8-bit DEM reference case. The calibration method acheives slighty superior performance in simulation, but would be much harder to effectively implement in silicon.

\subsection{Conclusion}

The ReQ method applies techniques developed for $\Delta \Sigma$ DACs to the ADC feedback path. The coarse and fine signals are reassigned using a first-order digital modulator, and then applied to the DEM and DAC circuits. The resulting system has slightly worse SNR performance than the calibration method previously discussed, but it does not require any special initialization or setup in order to work, and is potentially easier to implement.

This method adds significant delay to the feedback path, and adds some area and power consumption for the required circuitry. However, operation very near the reference case is achieved, and no additional steps or circuitry are required to use this method with higher-order $\Delta \Sigma$ modulators. 


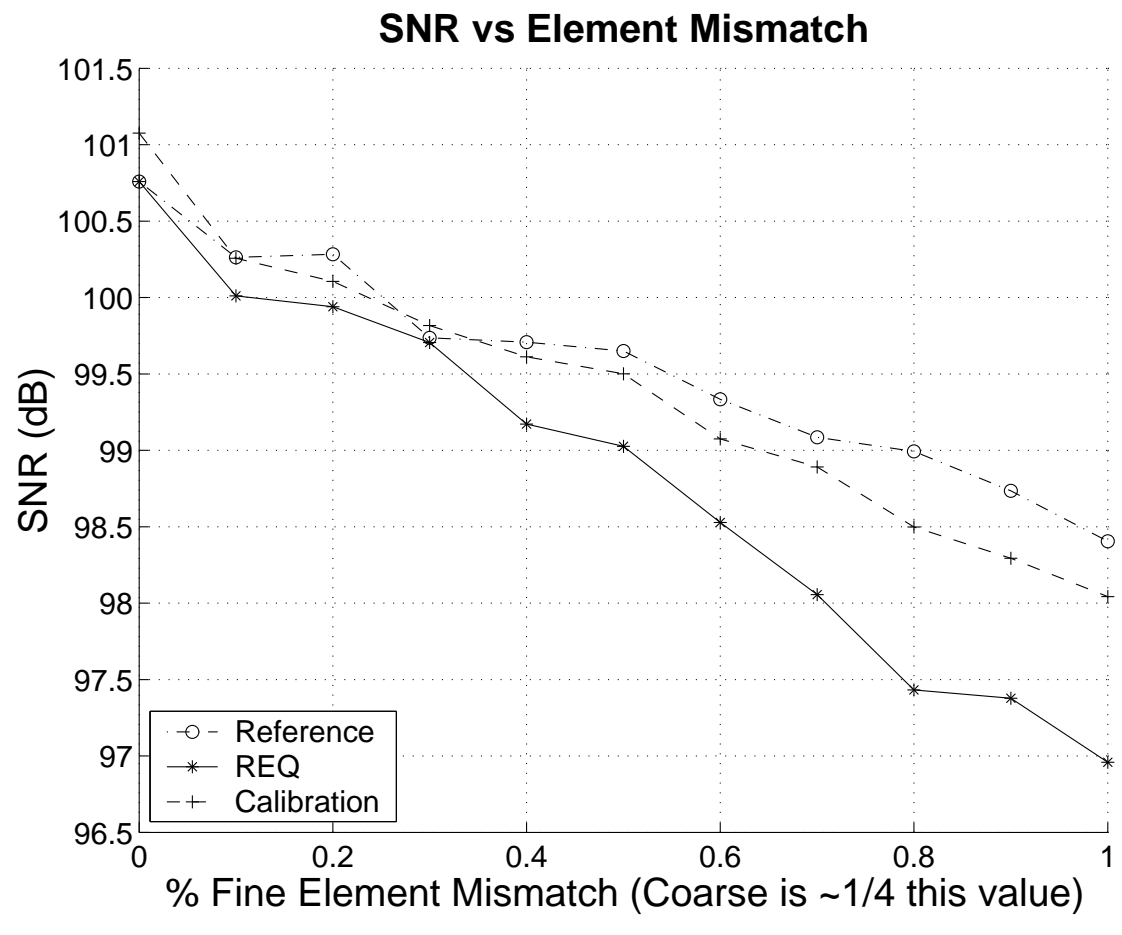

Figure 5.3: Simulated Results for ReQ System 


\section{Chapter 6}

\section{Circuit Design}

Both the calibration and requantization methods previously discussed are successful and efficient methods to implement a segmented DEM architecture. The physical realization of the calibration method depends on analog circuits to apply the calibration. The ReQ method, however, is completely digital in its implementation. As such, the physical operation of the ReQ method should be closer to its simulated performance than that of the calibration method. For this reason, the ReQ method was chosen as the solution for the current research application. This chapter presents the circuit designs to implement the ReQ method in silicon.

\subsection{Circuit Overviews}

The ReQ circuits presented in this chapter are designed to meet the following system specifications in a TSMC $0.25 \mu \mathrm{m}$ process: fourth-order $\Delta \Sigma \mathrm{ADC}, 50 \mathrm{MHz}$ clock rate, $N_{C}=4$, and $N_{F}=4$. Straight-forward designs were used (circuits without many speed optimizations) to see if standard circuits could be used to meet the time delay constraints. Due to the lack of available synthesis tools, the designs were done by hand, instead of using RTC. The overall block diagram of the feedback path is shown in Figure 6.1. Each block will be explained in turn, and full schematics for each can be found in Appendix A.

\subsubsection{Encoder}

The first blocks in the feedback path are the two encoders. These identical blocks first convert the thermometer code output of the ADC quantizer into a "one-hot" signal (a signal in which only one wire is asserted at any given time). In order to do this, the circuitry 


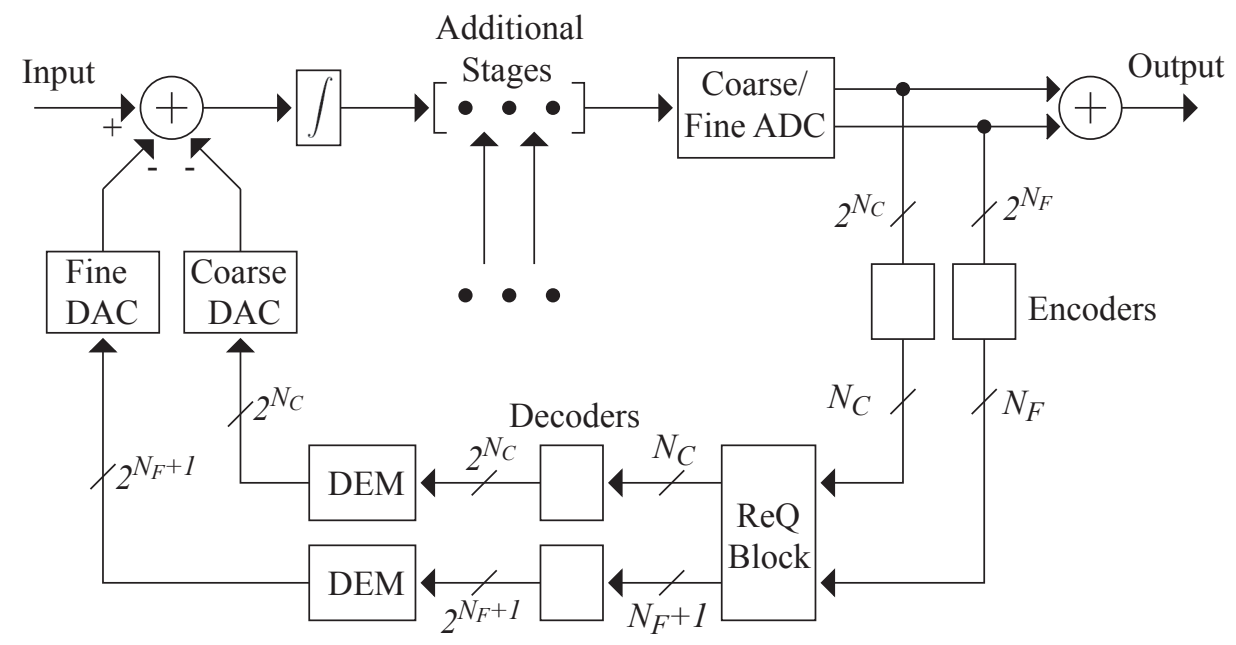

Figure 6.1: Overall Block Diagram of the Feedback Path for the ReQ Method

looks for the edge of the thermometer code - a sequence of "0 01 ". A three-input detection allows the circuit to eliminate a spurious code of "1 01 " (referred to as a "bubble"). The circuit is comprised of 3-input nor gates and inverters. Figure 6.2 shows this part of the design.

After this, the encoders convert the "one-hot" signals to binary. Since the output of these two blocks represent, respectively, the four most significant bits and four least significant bits of the 2's complement binary number, the circuits are slightly different: the encoder block for the most significant bits has an extra inverter on the MSB to make it output 2's complement signed numbers. Figure 6.3 shows the circuit design for the encoder handling the most significant bits, and Figure 6.4 shows the circuit design for the encoder handling the least significant bits. The circuits were designed using weak p-type pull-up devices to hold the outputs high, unless pulled low by strong n-type devices controlled by the input signals.

\subsubsection{ReQ Block}

The ReQ circuit (see Figure 6.5) is comprised of two 8-bit subtractors, a 5-bit subtractor, and an 8-bit register. The lower right part of Figure 5.1 shows a block diagram view 

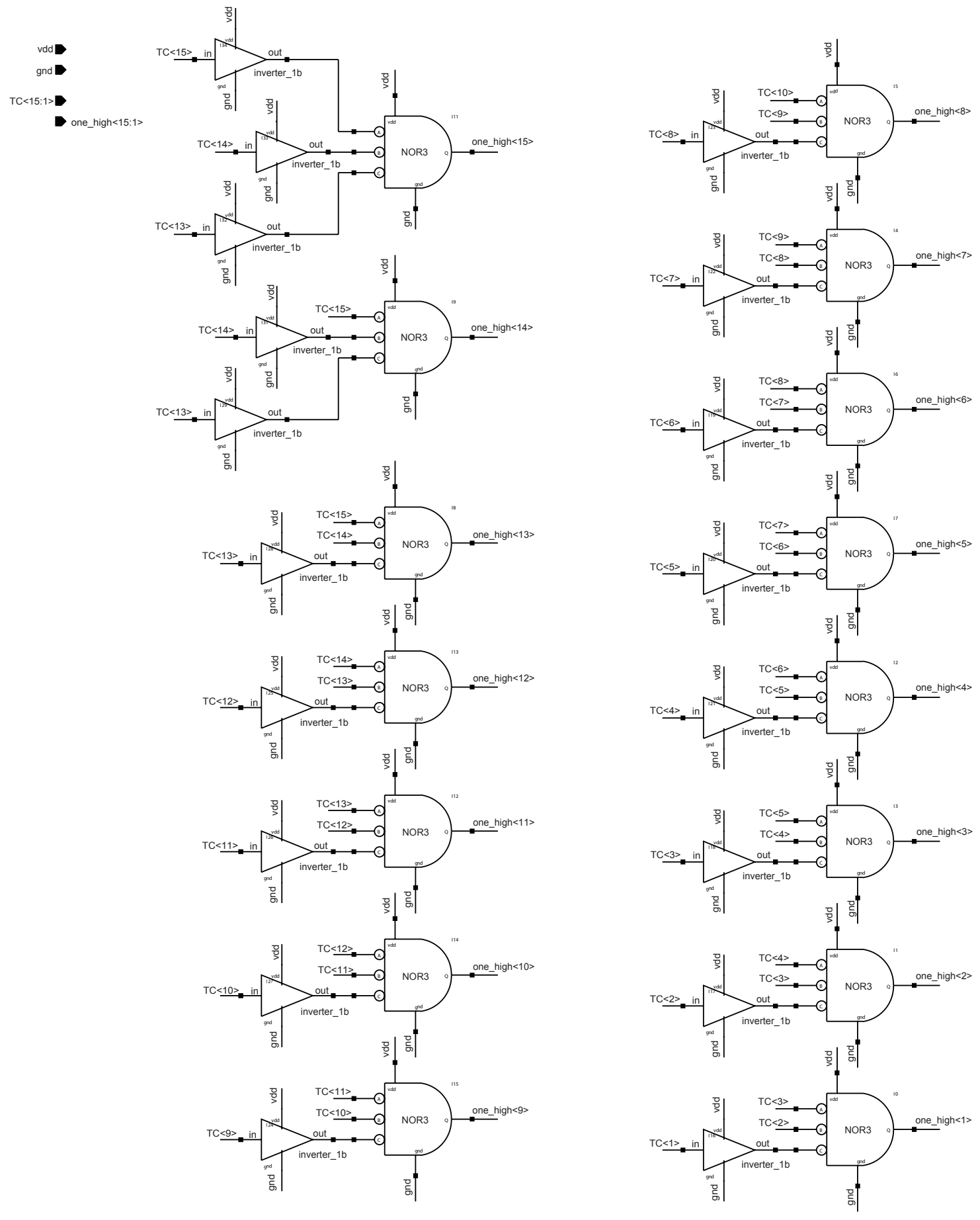

Figure 6.2: Bubble Decode Circuitry 


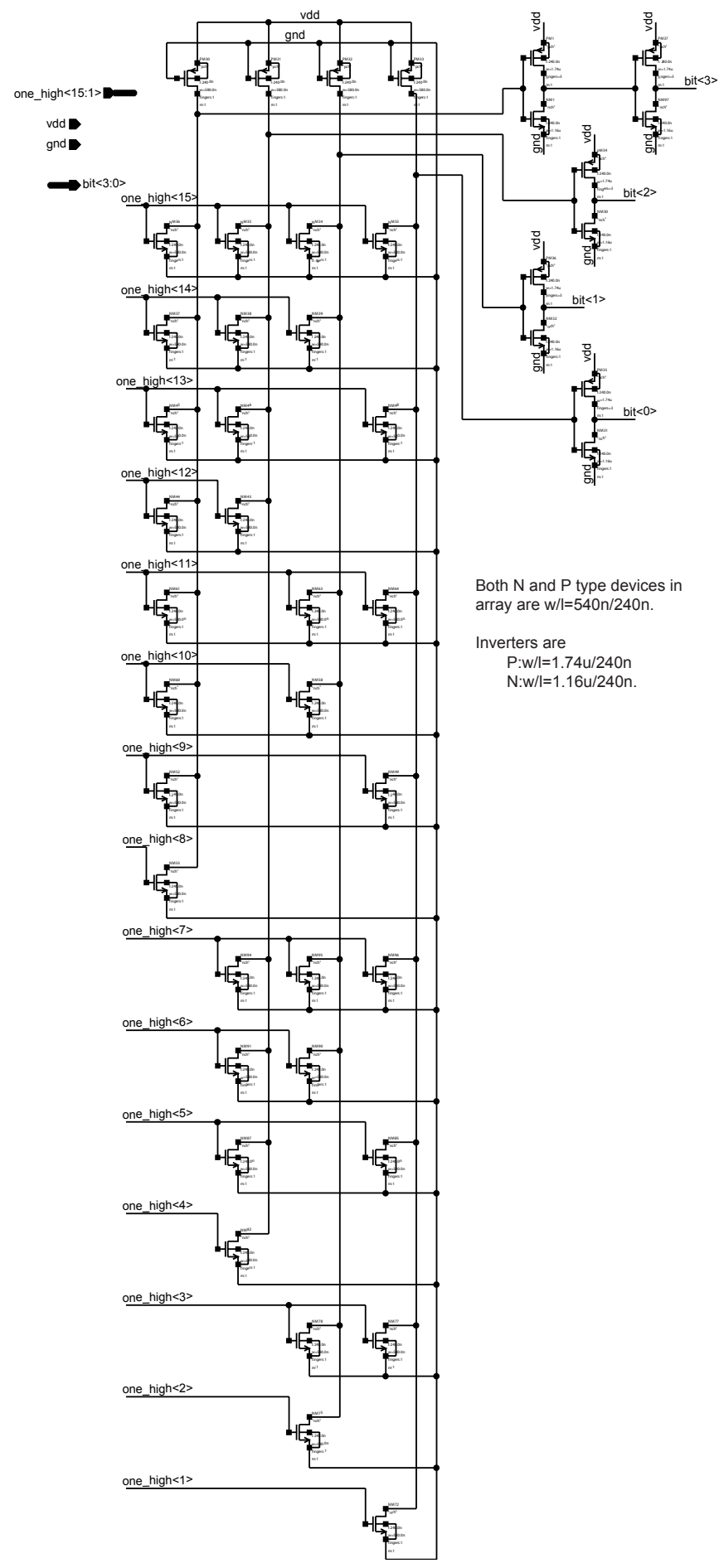

Figure 6.3: Encoder Circuit for the MSBs 


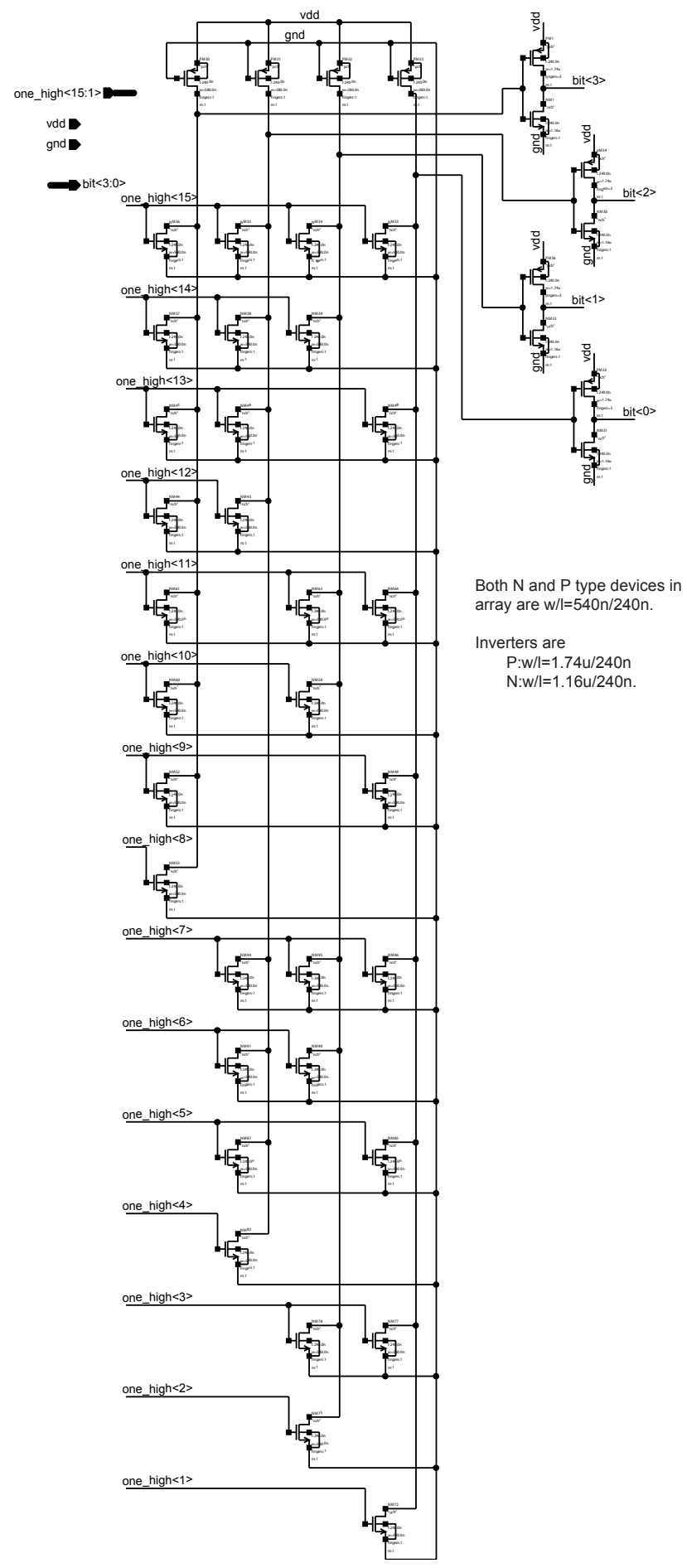

Figure 6.4: Encoder Circuit for the LSBs 
of the ReQ. The path including the register and the two 8-bit subtractors makes up a firstorder digital modulator to form a new noise-shaped coarse signal, and the 5-bit subtractor is used to form the new noise-shaped fine signal.

The subtractors are a simple ripple-carry design. Since they are only 8 or 5 bits wide, the potential speedup of carry lookahead circuits is small, and does not seem to offset the drawback of the increased size and power requirements. The basic full adder circuit is shown in Figure 6.6.

The D-flip-flops are a standard design, such as can be found in textbooks and web tutorials. Each flip-flop is made up of two anti-parallel inverters connected in a loop, with pass gates to break the feedback and connect the input signal when the controlling clock is high. Register setup and hold times are difficult to simulate without accurate transistor models, so care was taken to allow a generous amount of time for setup and hold. The circuit used in this design is shown in Figures 6.7 and 6.8 .

\subsubsection{Decode Logic}

The decode logic is a collection of complex gates to realize the conversion from 4or 5-bit binary to thermometer code. The logic was generated by hand using standard logic tables and k-maps. As an example, Table 6.1 shows the desired operation of the 4-bit binary-to-thermometer code conversion. The table for the 5-bit binary-to-thermometer code conversion follows the same pattern.

Since larger devices require more power, the transistors in the complex gates are all minimum size. This slows down the logic somewhat, but it is still fast enough for this application. Figures 6.9 and 6.10 show the schematics of the two decoder blocks.

\subsubsection{DEM Logic}

The DEM logic was designed using the methods presented in [13]. Figure 6.11 shows the basic operational blocks of the DEM logic for the coarse path: the rotate block and the rotate control. The rotate control logic takes the binary output signal from the ReQ logic and adds it to the current value stored in the pointer register. Since the current input code 


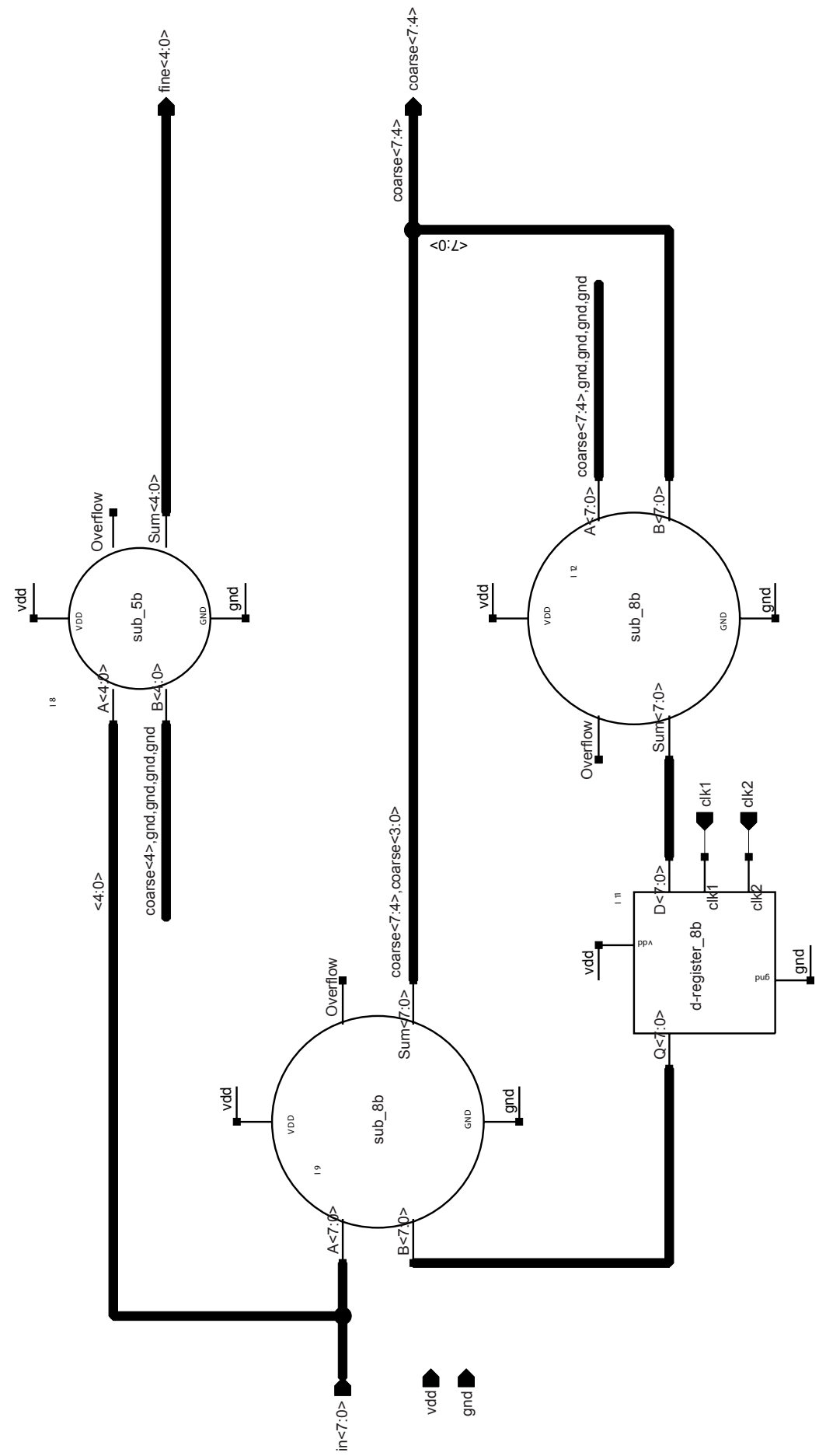

Figure 6.5: ReQ Circuit 


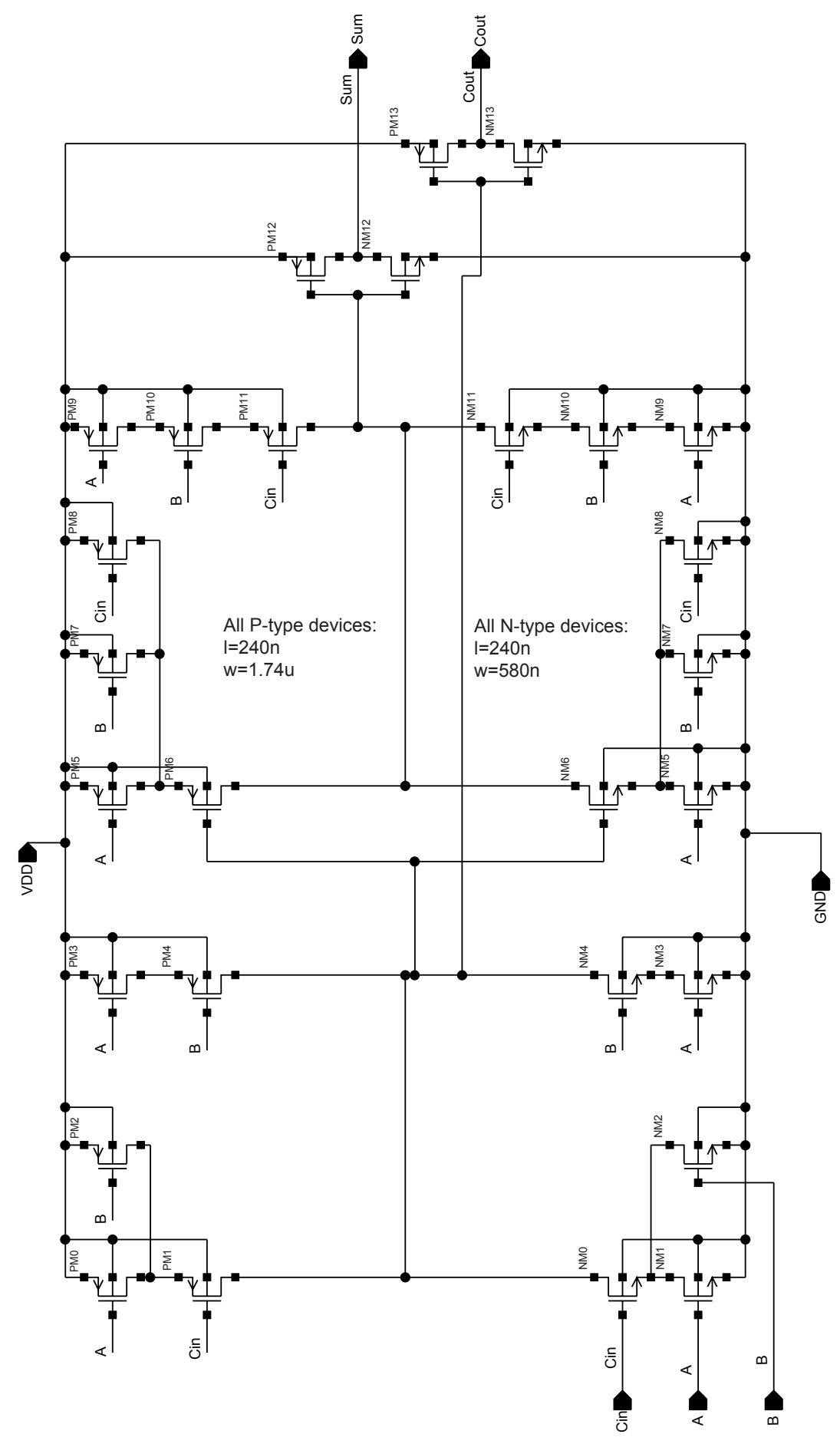

Figure 6.6: Full Adder Circuit 


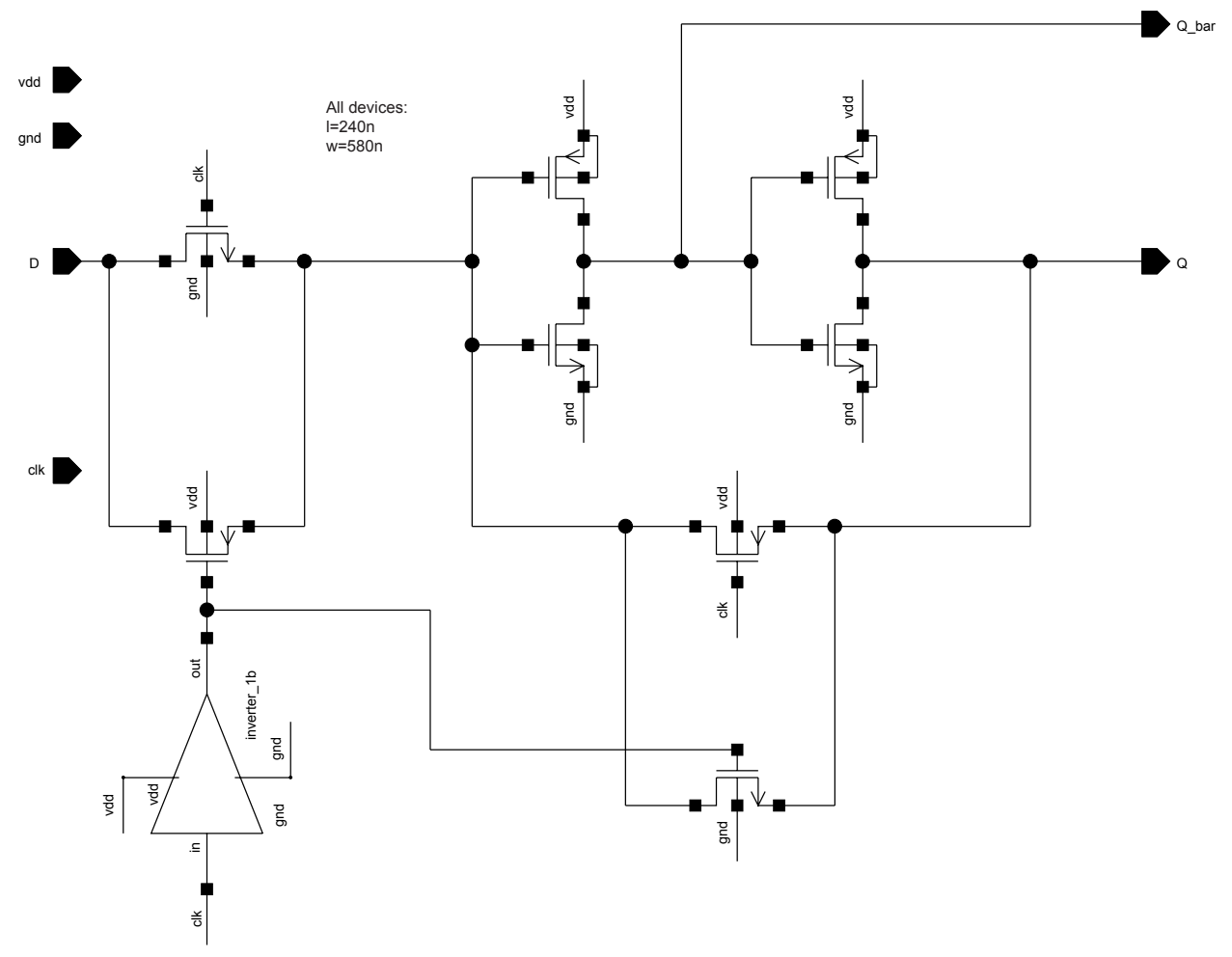

Figure 6.7: D-Latch Circuit

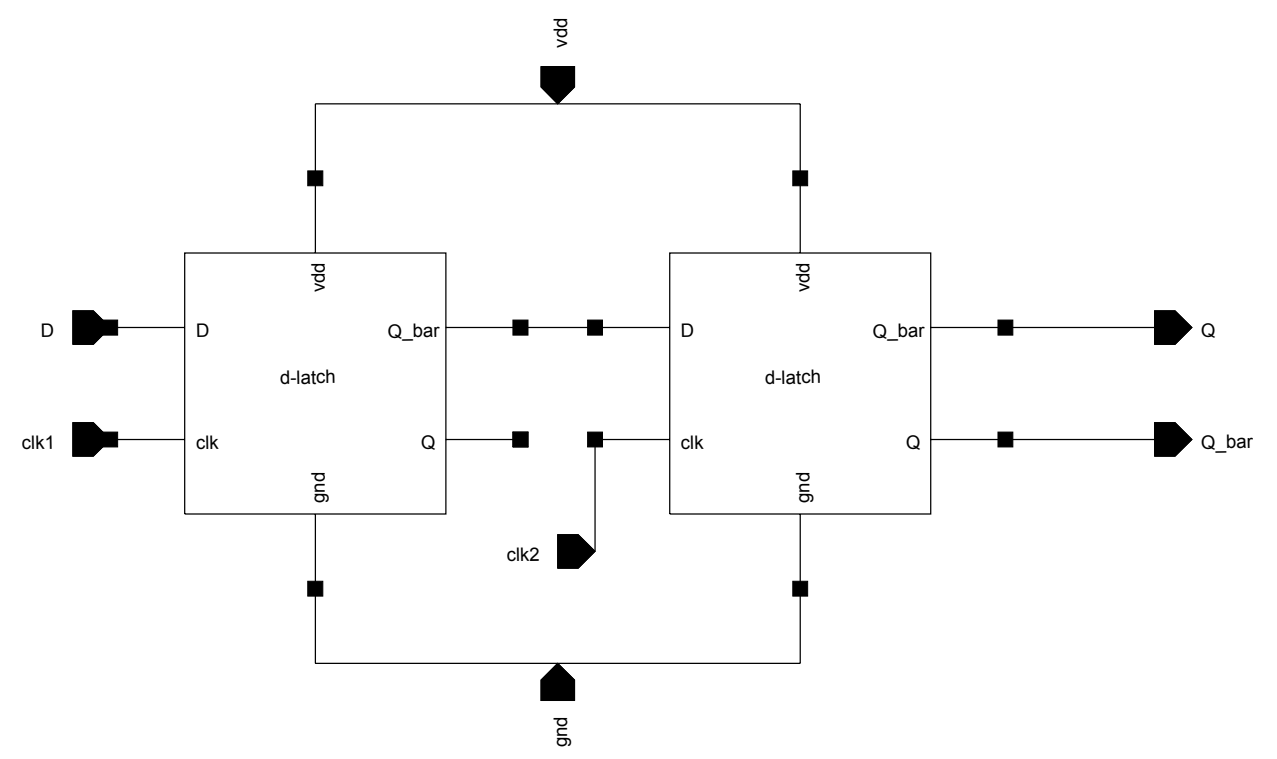

Figure 6.8: D-Register Circuit 


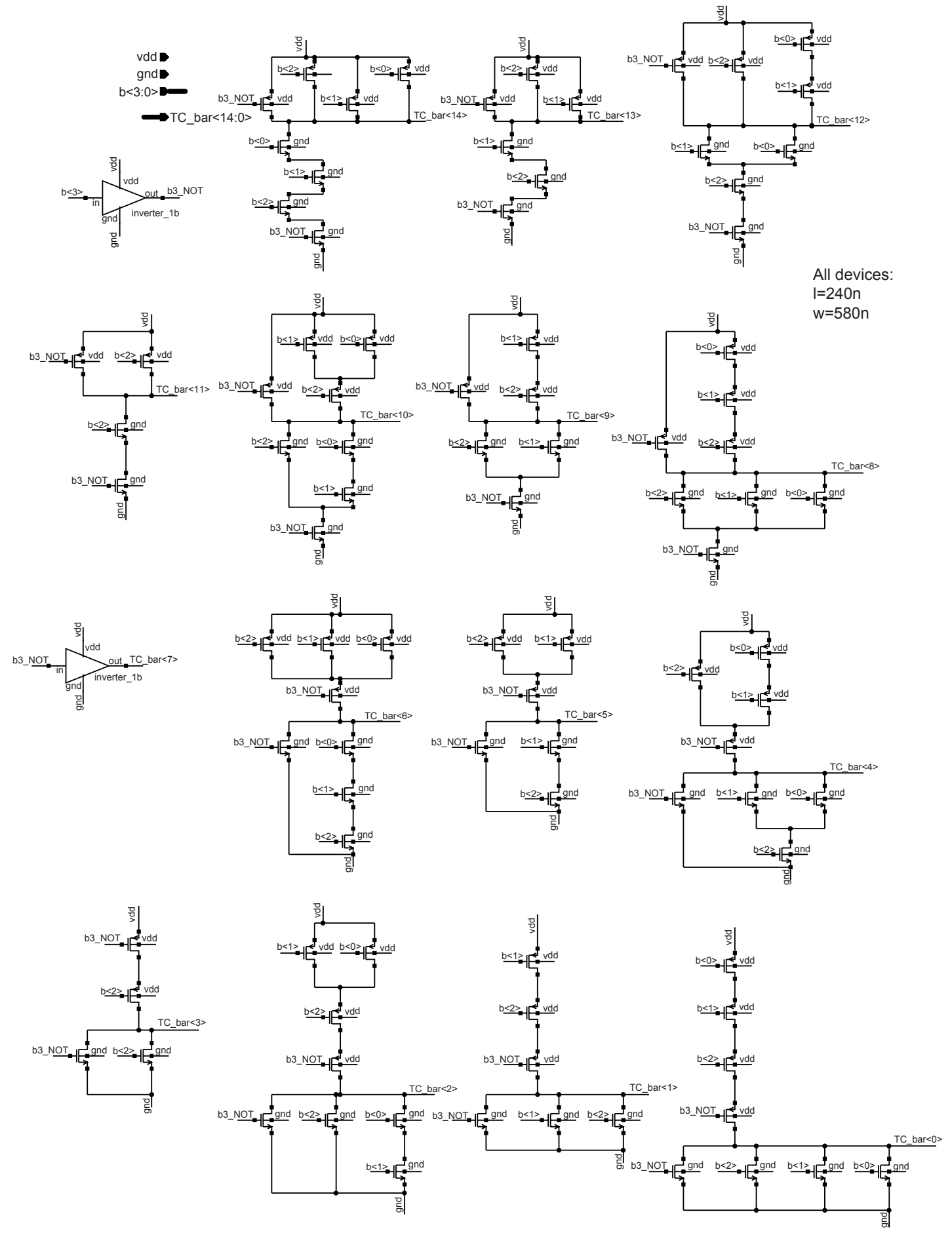

Figure 6.9: 4-Bit Binary-to-Thermometer Decoder Circuit 


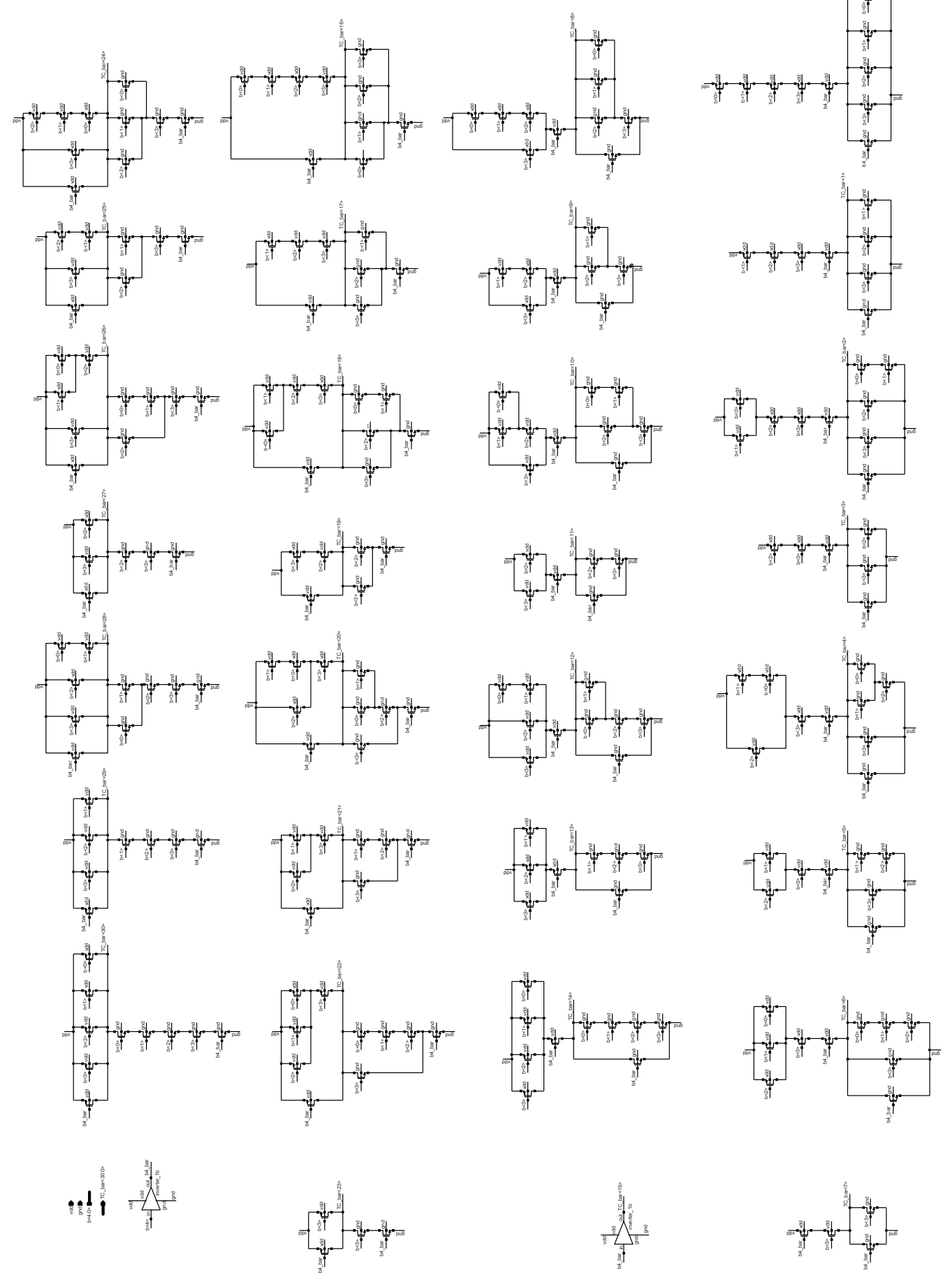

Figure 6.10: 5-Bit Binary-to-Thermometer Decoder Circuit 
Table 6.1: Logic Table for 4-Bit Binary-to-Thermometer Code Conversion

\begin{tabular}{|cccc|ccccccccccccccc|}
\hline $\mathrm{b} 3$ & $\mathrm{~b} 2$ & $\mathrm{~b} 1$ & $\mathrm{~b} 0$ & 14 & 13 & 12 & 11 & 10 & 9 & 8 & 7 & 6 & 5 & 4 & 3 & 2 & 1 & 0 \\
\hline 0 & 1 & 1 & 1 & 1 & 1 & 1 & 1 & 1 & 1 & 1 & 1 & 1 & 1 & 1 & 1 & 1 & 1 & 1 \\
0 & 1 & 1 & 0 & 0 & 1 & 1 & 1 & 1 & 1 & 1 & 1 & 1 & 1 & 1 & 1 & 1 & 1 & 1 \\
0 & 1 & 0 & 1 & 0 & 0 & 1 & 1 & 1 & 1 & 1 & 1 & 1 & 1 & 1 & 1 & 1 & 1 & 1 \\
0 & 1 & 0 & 0 & 0 & 0 & 0 & 1 & 1 & 1 & 1 & 1 & 1 & 1 & 1 & 1 & 1 & 1 & 1 \\
0 & 0 & 1 & 1 & 0 & 0 & 0 & 0 & 1 & 1 & 1 & 1 & 1 & 1 & 1 & 1 & 1 & 1 & 1 \\
0 & 0 & 1 & 0 & 0 & 0 & 0 & 0 & 0 & 1 & 1 & 1 & 1 & 1 & 1 & 1 & 1 & 1 & 1 \\
0 & 0 & 0 & 1 & 0 & 0 & 0 & 0 & 0 & 0 & 1 & 1 & 1 & 1 & 1 & 1 & 1 & 1 & 1 \\
0 & 0 & 0 & 0 & 0 & 0 & 0 & 0 & 0 & 0 & 0 & 1 & 1 & 1 & 1 & 1 & 1 & 1 & 1 \\
1 & 1 & 1 & 1 & 0 & 0 & 0 & 0 & 0 & 0 & 0 & 0 & 1 & 1 & 1 & 1 & 1 & 1 & 1 \\
1 & 1 & 1 & 0 & 0 & 0 & 0 & 0 & 0 & 0 & 0 & 0 & 0 & 1 & 1 & 1 & 1 & 1 & 1 \\
1 & 1 & 0 & 1 & 0 & 0 & 0 & 0 & 0 & 0 & 0 & 0 & 0 & 0 & 1 & 1 & 1 & 1 & 1 \\
1 & 1 & 0 & 0 & 0 & 0 & 0 & 0 & 0 & 0 & 0 & 0 & 0 & 0 & 0 & 1 & 1 & 1 & 1 \\
1 & 0 & 1 & 1 & 0 & 0 & 0 & 0 & 0 & 0 & 0 & 0 & 0 & 0 & 0 & 0 & 1 & 1 & 1 \\
1 & 0 & 1 & 0 & 0 & 0 & 0 & 0 & 0 & 0 & 0 & 0 & 0 & 0 & 0 & 0 & 0 & 1 & 1 \\
1 & 0 & 0 & 1 & 0 & 0 & 0 & 0 & 0 & 0 & 0 & 0 & 0 & 0 & 0 & 0 & 0 & 0 & 1 \\
1 & 0 & 0 & 0 & 0 & 0 & 0 & 0 & 0 & 0 & 0 & 0 & 0 & 0 & 0 & 0 & 0 & 0 & 0 \\
\hline
\end{tabular}

is used to calculate the control signal for the following cycle, this computation is performed outside the loop of the modulator and has no bearing on the critical path of the overall ADC.

The current value in the pointer register is used as the control signal to the rotate block, which rotates the thermometer-coded output from the decoder a fixed number of digit positions in response to the pointer control value. The rotate block is made up of 4 separate rotate cells that each conditionally rotate by a fixed amount, depending on the pointer value. For example, the "rotate 2" block passes the input unchanged to its output if bit 1 of the pointer control signal is not asserted, or rotates the input signal by 2 positions if the control bit is asserted. In other words, the "rotate 2" cell passes bit 0 of its input to bit 2 of its output, bit 1 of its input to bit 3 of its output, and so on, as illustrated on the right hand side of Figure 6.11. Each rotate cell is designed with transmission gates. The Figure actually shows the block diagram for the 4-bit coarse case. The 5-bit fine DEM has a 5-bit pointer and an extra rotate cell that rotates by 16 . Figure 6.12 shows the DEM schematics. 


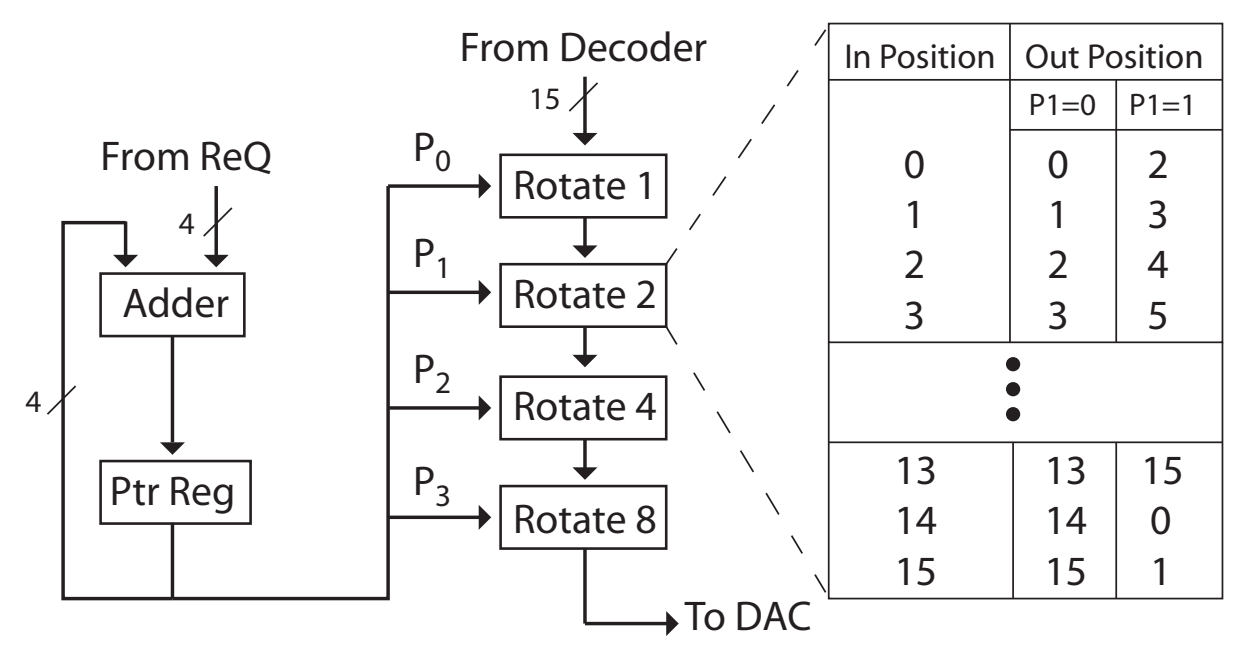

Figure 6.11: Block Diagram of DEM Circuitry

\subsection{Circuit Simulations}

The target clock rate for this design is $50 \mathrm{MHz}$. Due to the design used for the twostep quantizer and integrators, the computations in the digital feedback must be completed during one-half clock cycle. This requires a worst-case delay of less than $10 \mathrm{~ns}$. The "feedback path" includes the encoder blocks, the ReQ, the decode blocks, and the DEM logic. (A small amount of extra time must be allotted for the comparators in the internal quantizer at the beginning and the DACs at the end, so the actual time available is less than $10 \mathrm{~ns}$.) The critical path for the digital feedback involves the following: a 4-bit bubble decode, a 4-bit thermometer code-to-binary encoder, an 8-bit adder and a 5-bit adder in the ReQ block, a 5-bit binary-to-thermometer decoder, and a 31-line DEM consisting of 5 stages of pass gates. Achieving a transistor-level simulation in which all of these elements operated in the worst-case condition proved to be very difficult, as the resulting output from a worst-case simulation of some blocks did not allow for the worst-case simulation of connected blocks. To simulate an absolute maximum delay, simulations were run on each block separately, carefully loading each block to mimic its connected operation. Figures 6.13, 6.14, 6.15, 6.16, and 6.17 show timing diagrams for each of the blocks in the critical path, and Table 6.2 shows the overall results of these simulations. The overall delay should be acceptable; more 

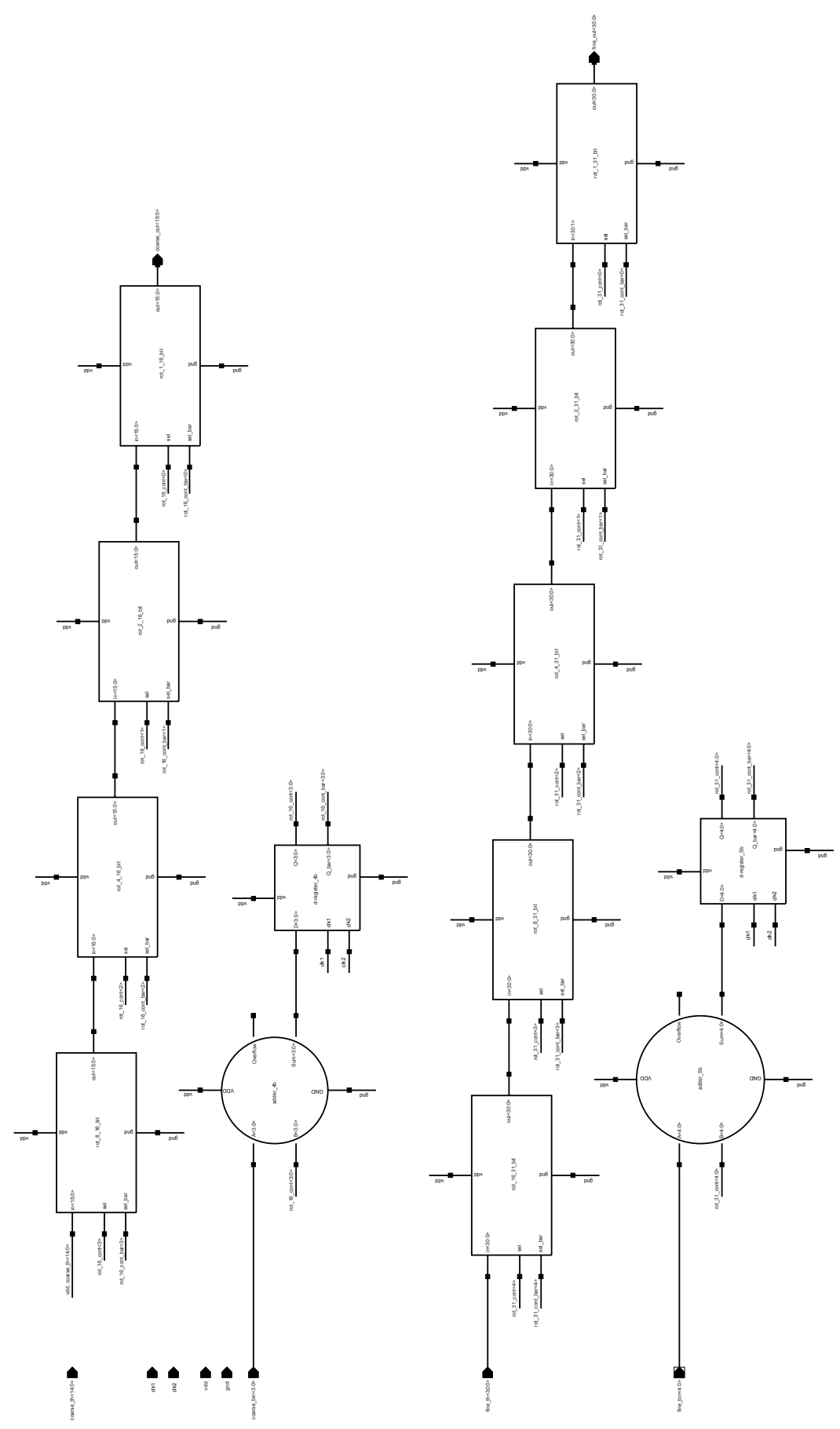

Figure 6.12: DEM Circuitry 
Table 6.2: Timing Results for the Critical Path

\begin{tabular}{|ll|c|rc|}
\hline & \multicolumn{2}{c|}{ Block } & Equivalent & \multicolumn{2}{c|}{ Worst- } \\
& Fane & Fan Out & Case Delay \\
\hline 1) & Bubble Decode & 1 inverter & 0.15 & $\mathrm{~ns}$ \\
2) & Therm-to-Bin Encoder & 8 inverters & 0.23 & $\mathrm{~ns}$ \\
$3)$ & 8-bit Subtractor & 25 inverters & 1.94 & $\mathrm{~ns}$ \\
$4)$ & 5-bit Subtractor & 35 inverters & 1.46 & $\mathrm{~ns}$ \\
$5)$ & $\begin{array}{c}\text { Bin-to-Therm Decoder } \\
\text { and 31-line DEM }\end{array}$ & $\begin{array}{c}\text { 5 layers of muxes } \\
\text { and 2 inverters }\end{array}$ & 2.50 & $\mathrm{~ns}$ \\
\hline \hline TOTAL: & & $\sim 6.28 \mathrm{~ns}$ \\
\hline
\end{tabular}

simulations that take into account delays of the analog components involved are needed to thoroughly verify this.

\subsection{Conclusion}

The circuits presented in this chapter are basic circuit designs such as one could find in basic textbooks. A few blocks, such as the decoders, were done as complex logic gates using basic design methods. The design has an overall worst-case delay of under 7 ns, which is less than the maximum allowable delay of $10 \mathrm{~ns}$. 


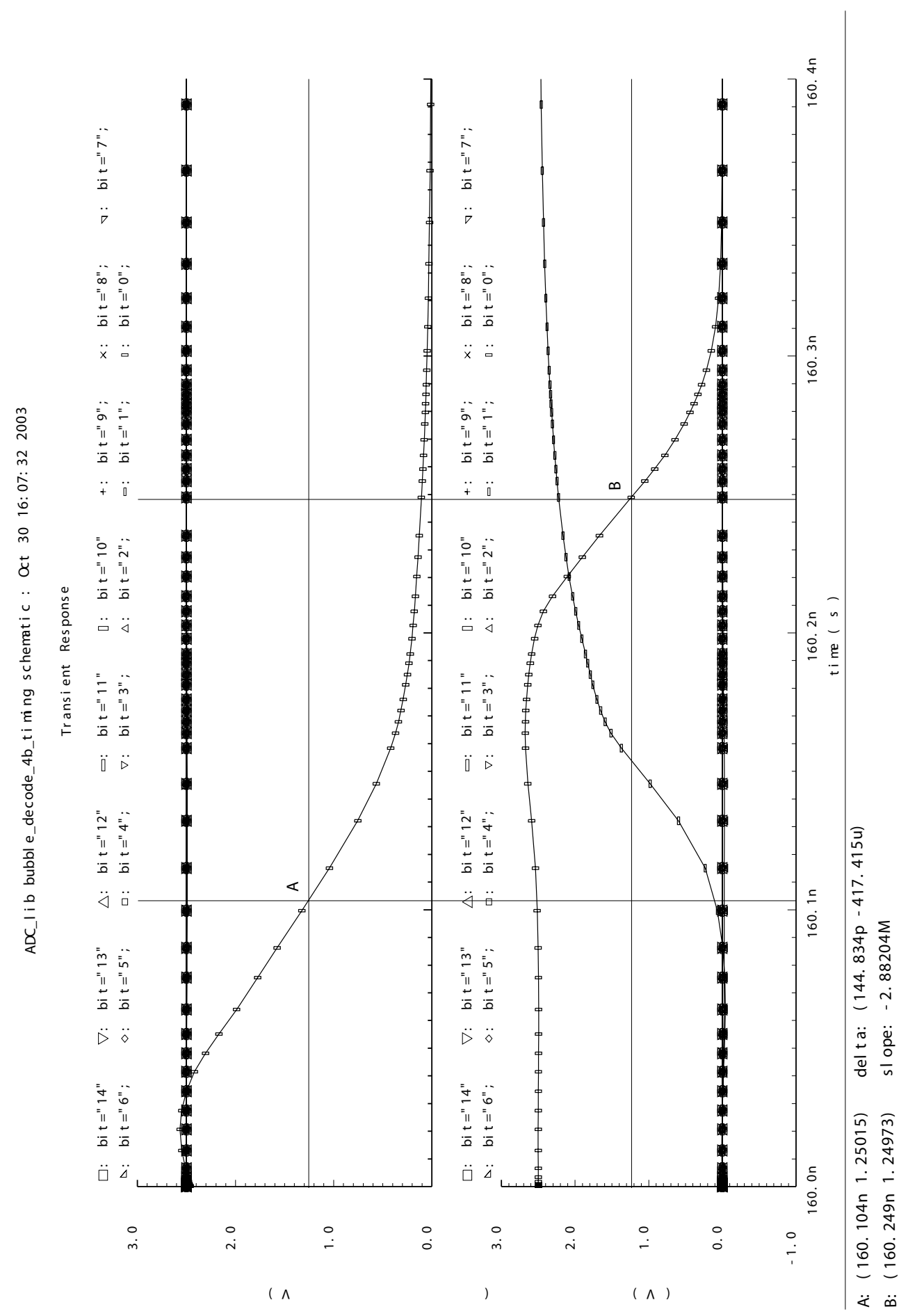

Figure 6.13: Timing Simulation for ReQ Bubble Decode Block 


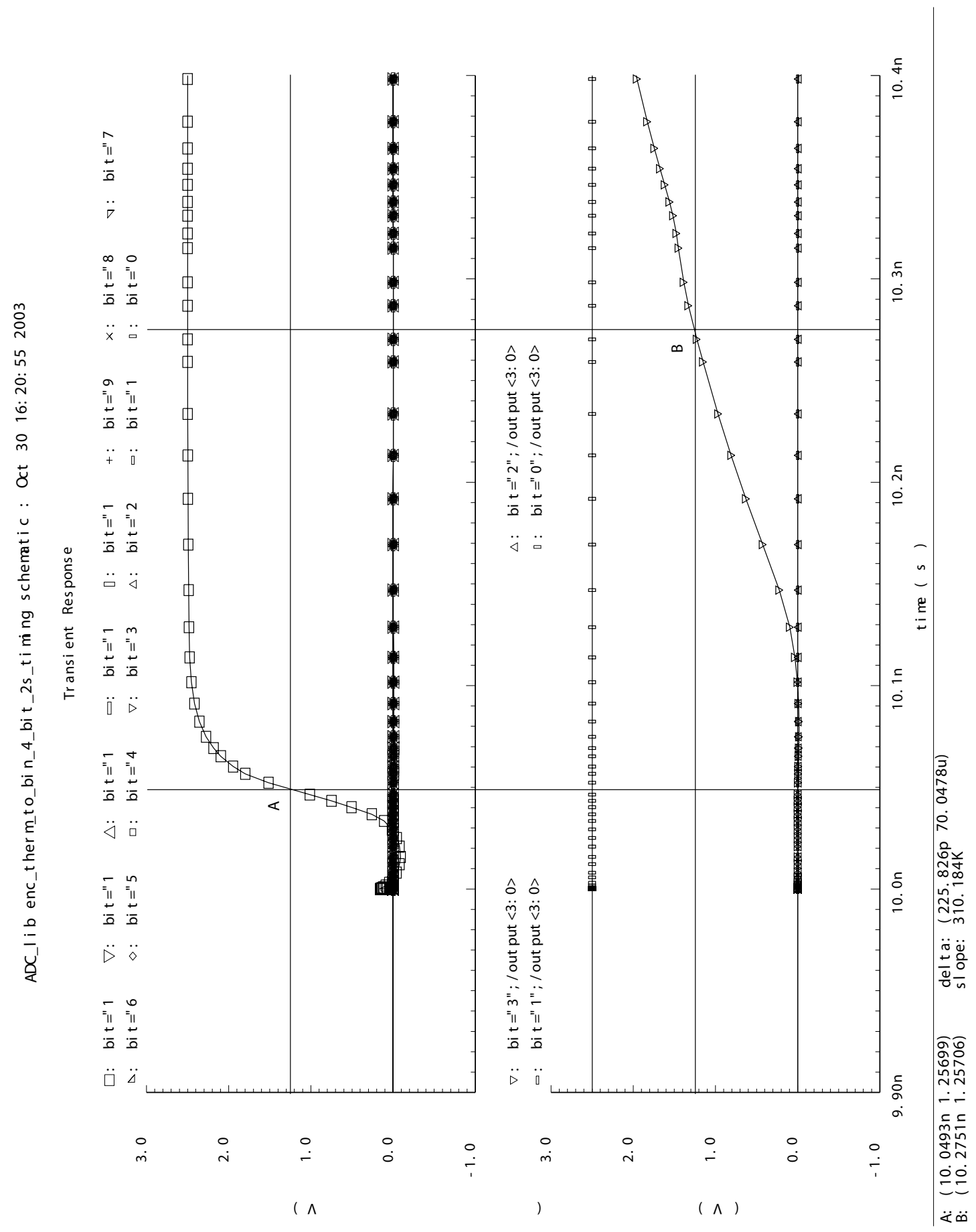

Figure 6.14: Timing Simulation for ReQ Encoder Block 


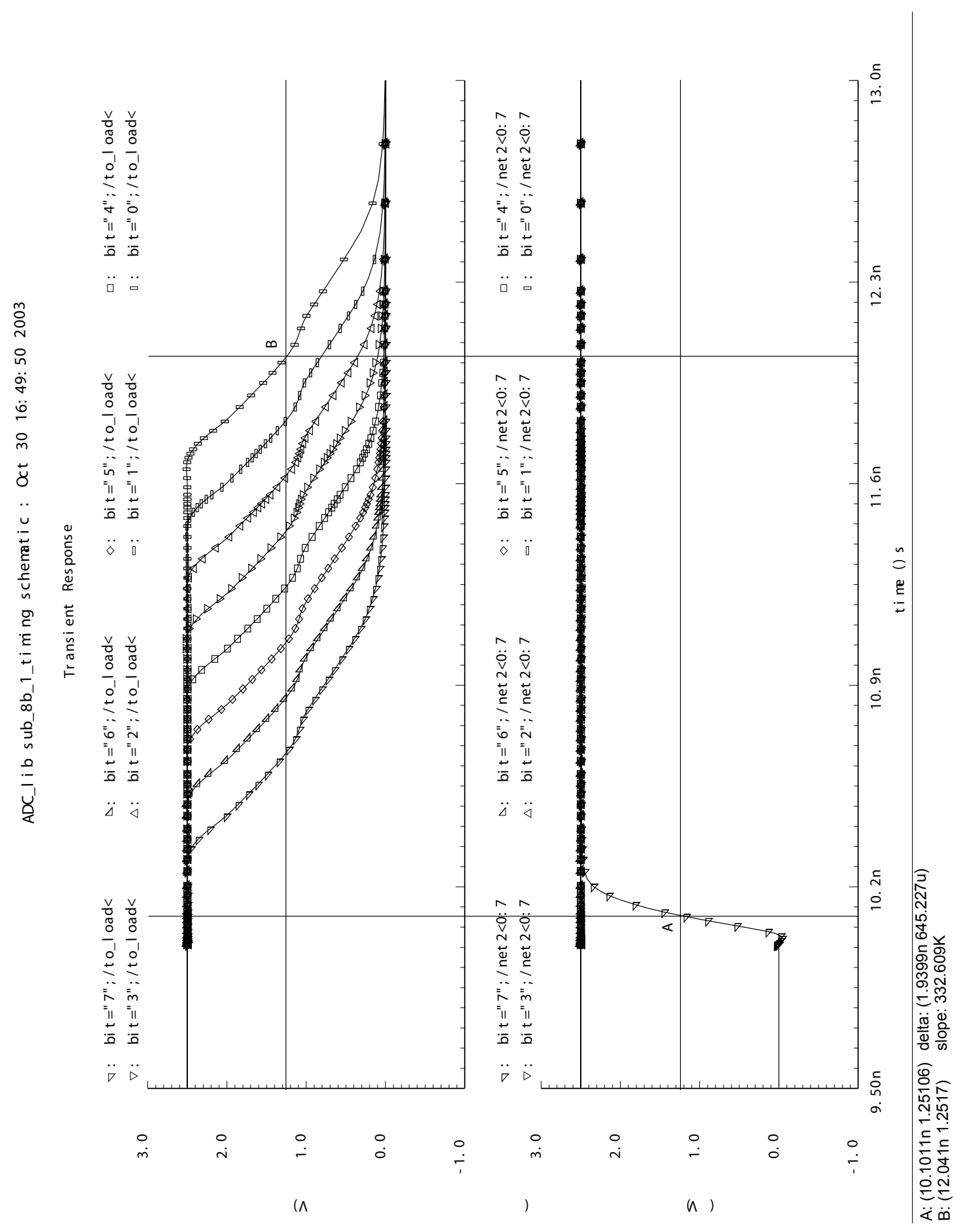

Figure 6.15: Timing Simulation for ReQ 8-bit Subtractor Block 


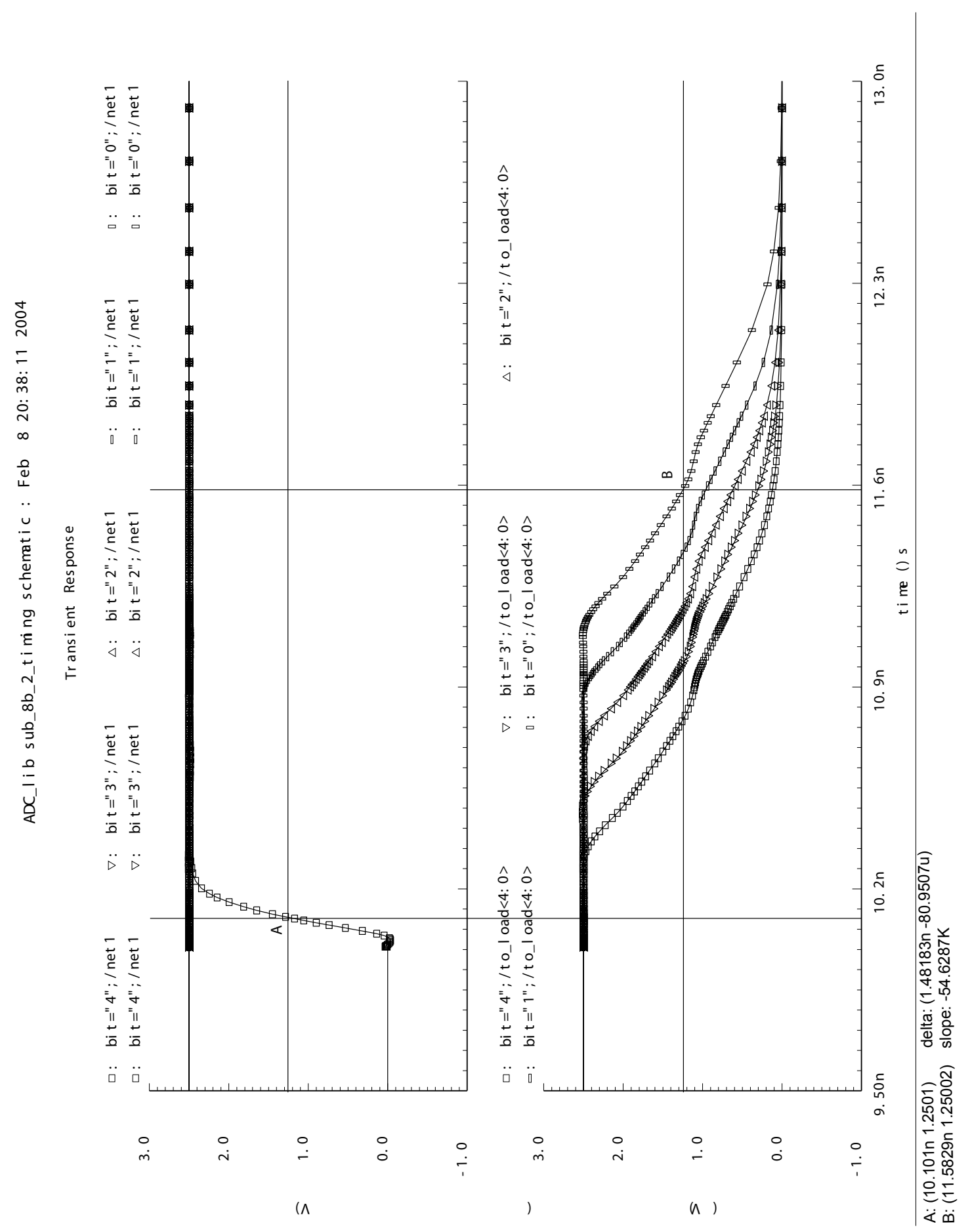

Figure 6.16: Timing Simulation for ReQ 5-bit Subtractor Block 


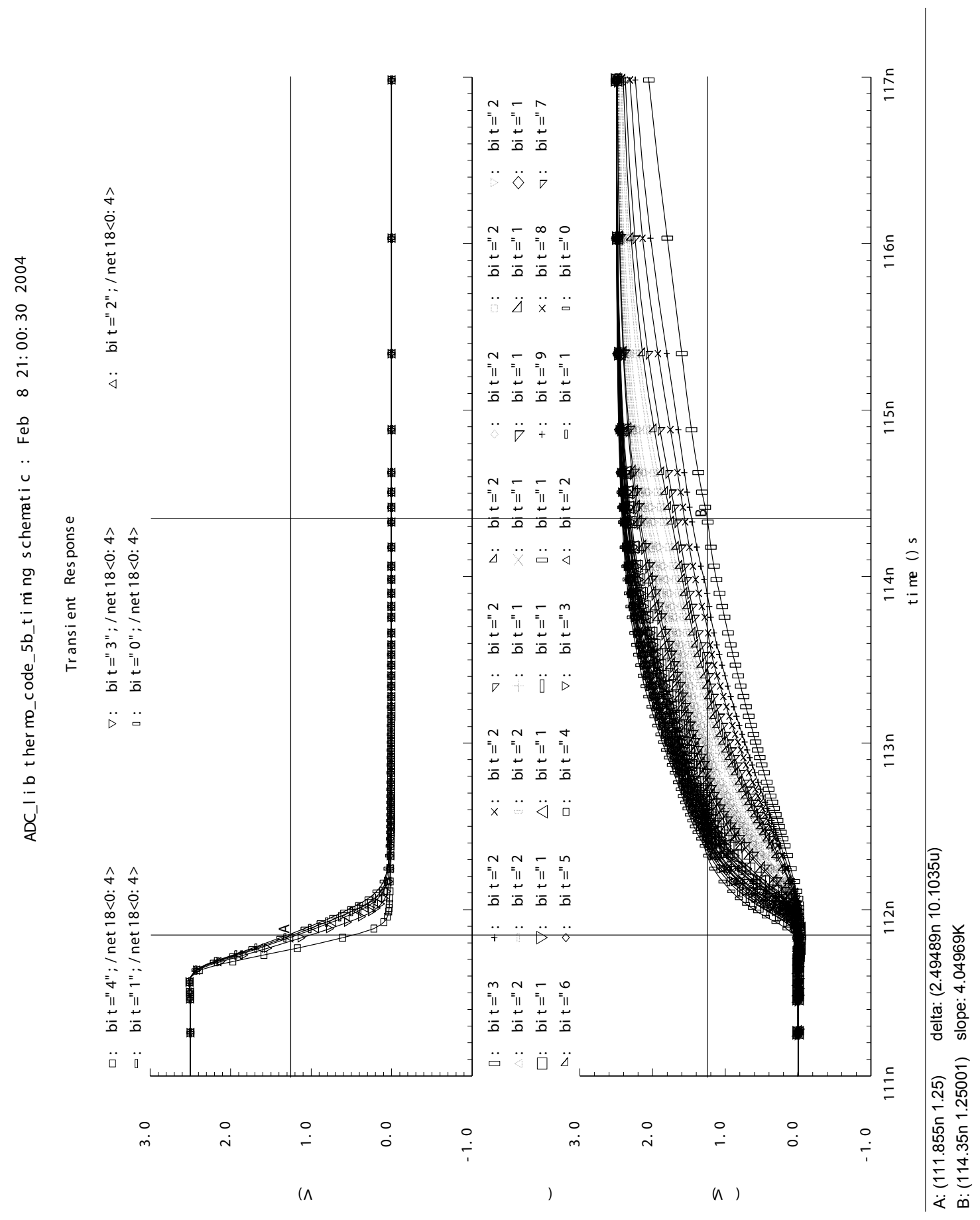

Figure 6.17: Timing Simulation for ReQ Decoder Block 


\section{Chapter 7}

\section{Conclusion}

\section{$7.1 \quad$ Contributions}

The intended contributions of this research are as follows:

- A new presentation and mathematical analysis of the problems involved with segmenting the digital word in a $\Delta \Sigma$ ADC feedback path.

- Design of two potential methods to overcome these problems: a calibration method and a requantization method.

- Simulations for these two potential solutions, along with an analysis and comparison.

- Designs for the circuits of the ReQ method to be used with a $50 \mathrm{MHz} \Delta \Sigma$ ADC design with 8-bit internal quantization and a desired output resolution of over 15 bits, along with timing and functional simulations.

A paper presenting this work has been accepted for publication at the 2004 IEEE International Symposium on Circuits and Systems [15].

\subsection{Summary of Results}

Chapter 3 presented that segmentation of the DAC structures in the feedback path of $\Delta \Sigma$ ADCs is desirable. It simplifies circuitry and allows for higher levels of internal quantization. However, the mismatch between the two DAC banks adds an additional source of error to the system, significantly reducing the effective SNR of the overall modulator. Two potential solutions were proposed: one to calibrate the DAC banks and remove the mismatch, 
and the other to requantize the segmented signals and frequency shape the error resulting from the mismatch away from the band of interest.

The calibration method described in Chapter 4 adds no extra logic to the feedback path of the $\Delta \Sigma \mathrm{ADC}$, insuring that the feedback path does not limit the overall clock speed. It does, however, add an initialization routine to set the calibration values, which must be run before regular operation can occur. This calibration routine must calibrate the DACs for each stage of the $\Delta \Sigma$ modulator, increasing the time required to perform calibration for high-order modulators. In the test case of a second-order modulator with an OSR of 20, an internal quantization of $(4+4)$-bit, and the coarse and fine percent mismatches scaled assuming a 16:1 size ratio between coarse and fine elements, the calibration method achieves an average SNR of $98 \mathrm{~dB}$ at a mismatch of $1 \%$. This is only $0.5 \mathrm{~dB}$ less than the SNR of the full 8-bit DEM reference case.

The ReQ method described in Chapter 5 does add logic to the feedback path, but it requires no initialization or startup routine. The critical path through the required logic is fairly short, and can be optimized to limit the effect of the feedback path logic on the overall clock speed. The DC offset inserted by the ReQ logic can be removed by a small change in the DAC structure, and the overall SNR of this method is acceptable, if a little worse than that of the calibration method. In the standard test case at $1 \%$ fine element mismatch, the ReQ method achieves an average SNR of about $96 \mathrm{~dB}$, which is only $2 \mathrm{~dB}$ less than the SNR of the full 8-bit DEM reference case.

The ReQ method scales better for use with higher-order modulators. It also has fewer implementation-related random factors to influence its actual operation in silicon, as it is completely digital. For these reasons, it is the chosen method to use for the specific $\Delta \Sigma$ design. The circuits were designed (discussed in Chapter 6, and shown in full in Appendix A to minimize power consumption while still meeting the timing goals. The total delay achieved through the feedback path was about 7 ns.

\subsection{Areas for Potential Future Research}

Some areas of suggested research on the material presented in this thesis are: 
- Further develop and verify the analysis of the DC offset introduced by the ReQ method. More specifically identify its source and how to avoid or more completely remove it, preferably using a digital method.

- Adapt a higher-order DEM algorithm that does not introduce significant additionally delay for use in the ADC. This would permit the use of a higher-order ReQ circuit and provide higher output resolution.

- Expand existing background calibration methods that calibrate DAC elements into one which will provide background calibration between the coarse and fine banks [14].

- Further optimize the digital circuitry of the $\Delta \Sigma$ feedback path. This includes higherorder or more efficient methods of performing the ReQ modulation, as well as more efficient circuits to implement all functions. 
Appendix 


\section{Appendix A}

\section{Complete Circuit Schematics}

\section{A.1 Top Level Schematic}

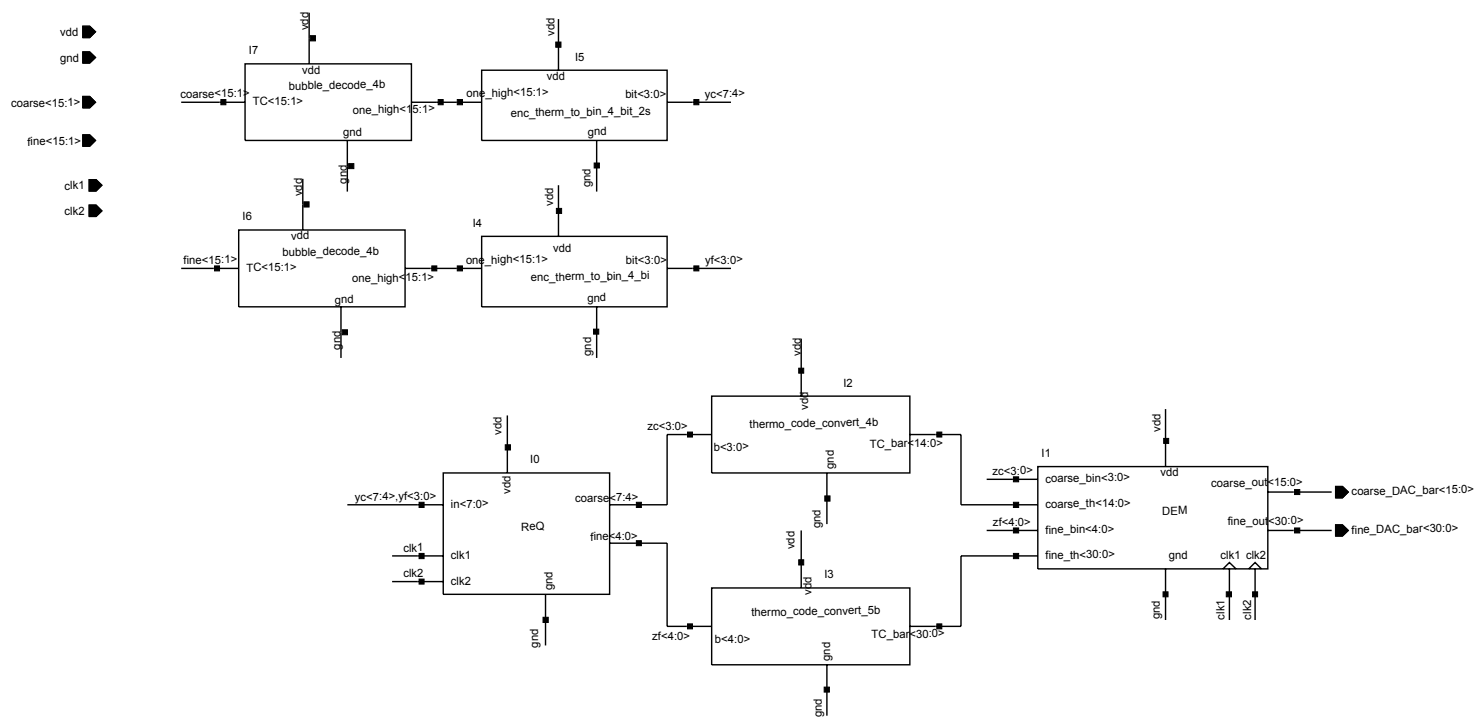

Figure A.1: Overall Schematic for Digital Feedback Path 


\section{A.2 Bubble Decode}
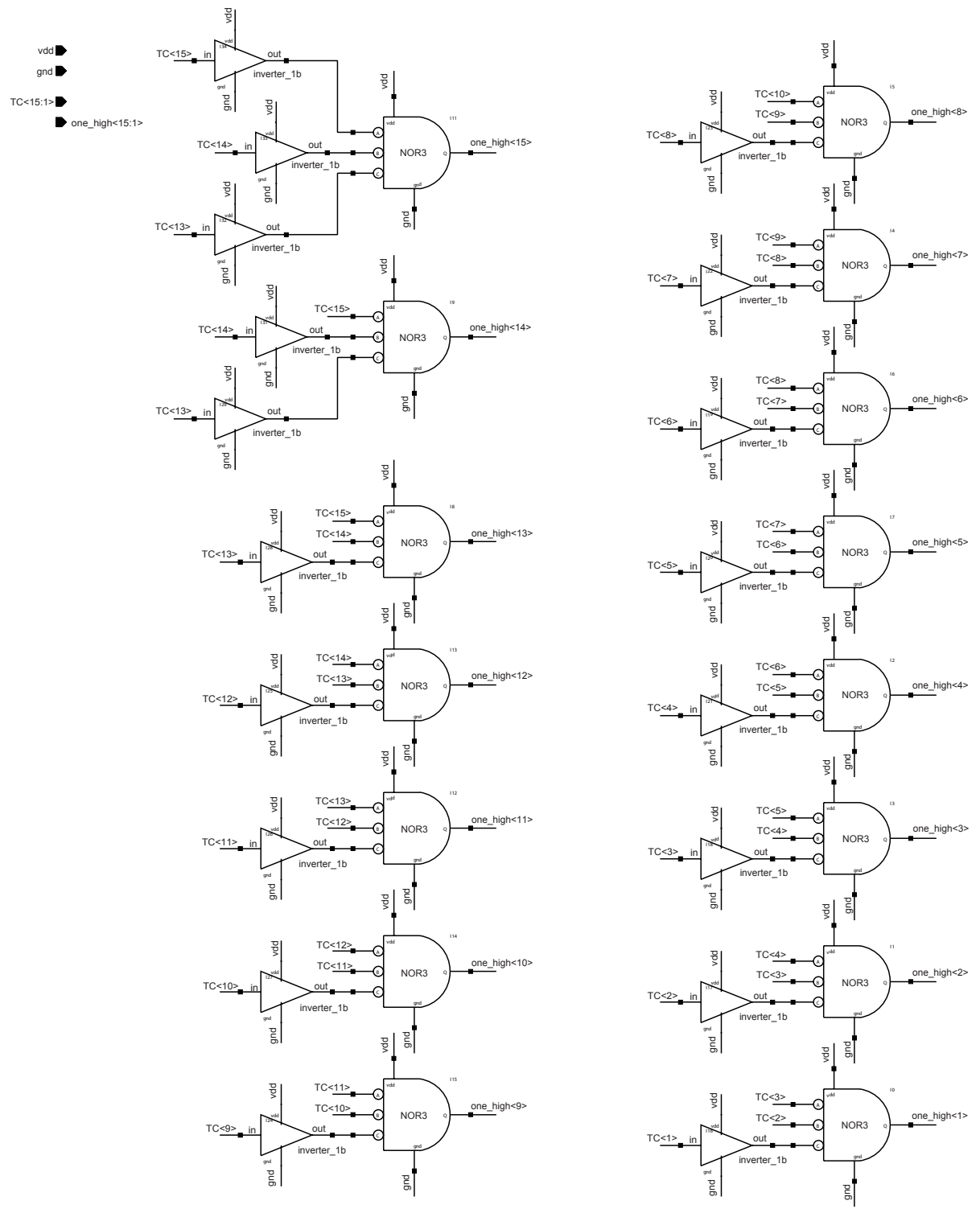

Figure A.2: Bubble Decode Logic 


\section{A.2.1 Inverter}

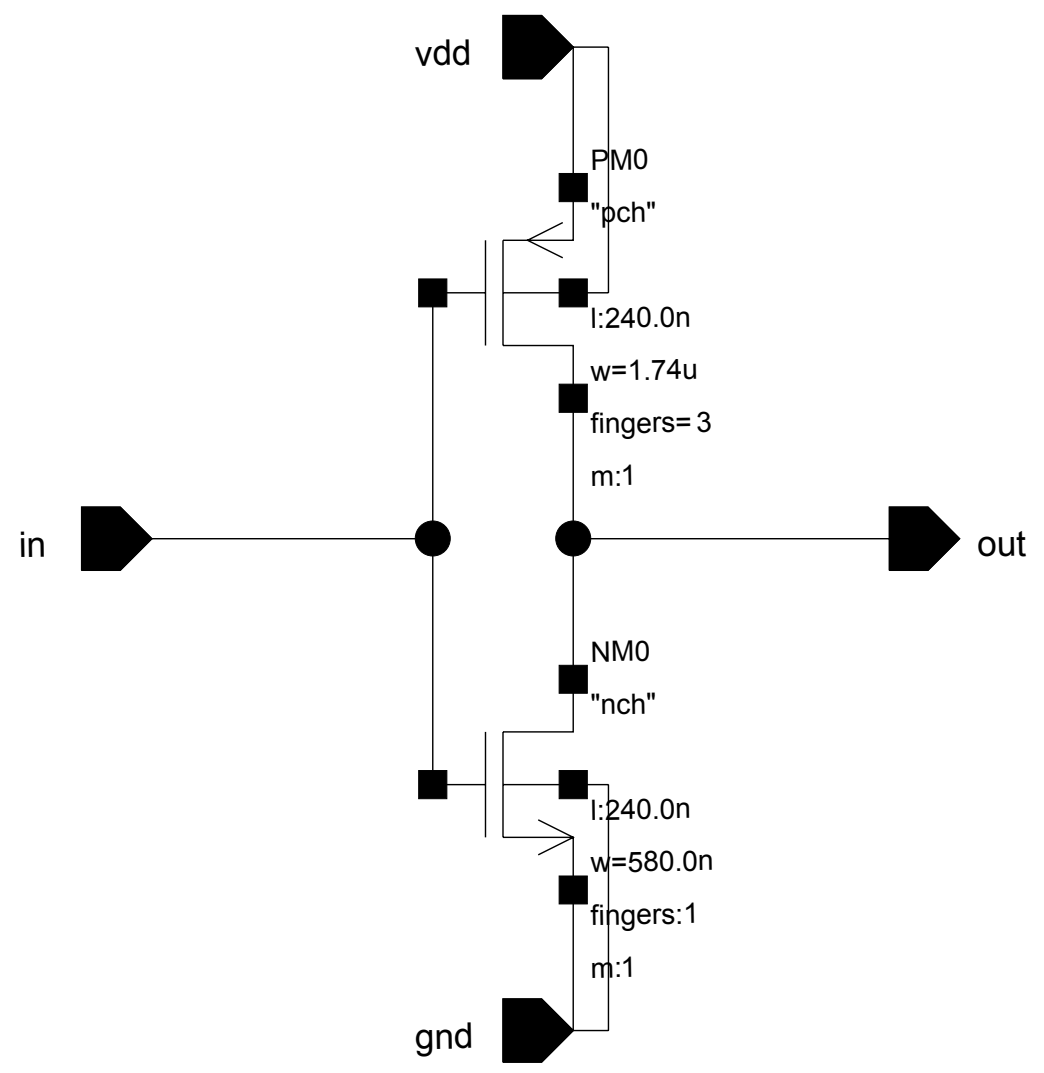

Figure A.3: Inverter 


\section{A.2.2 3 Input Nor}

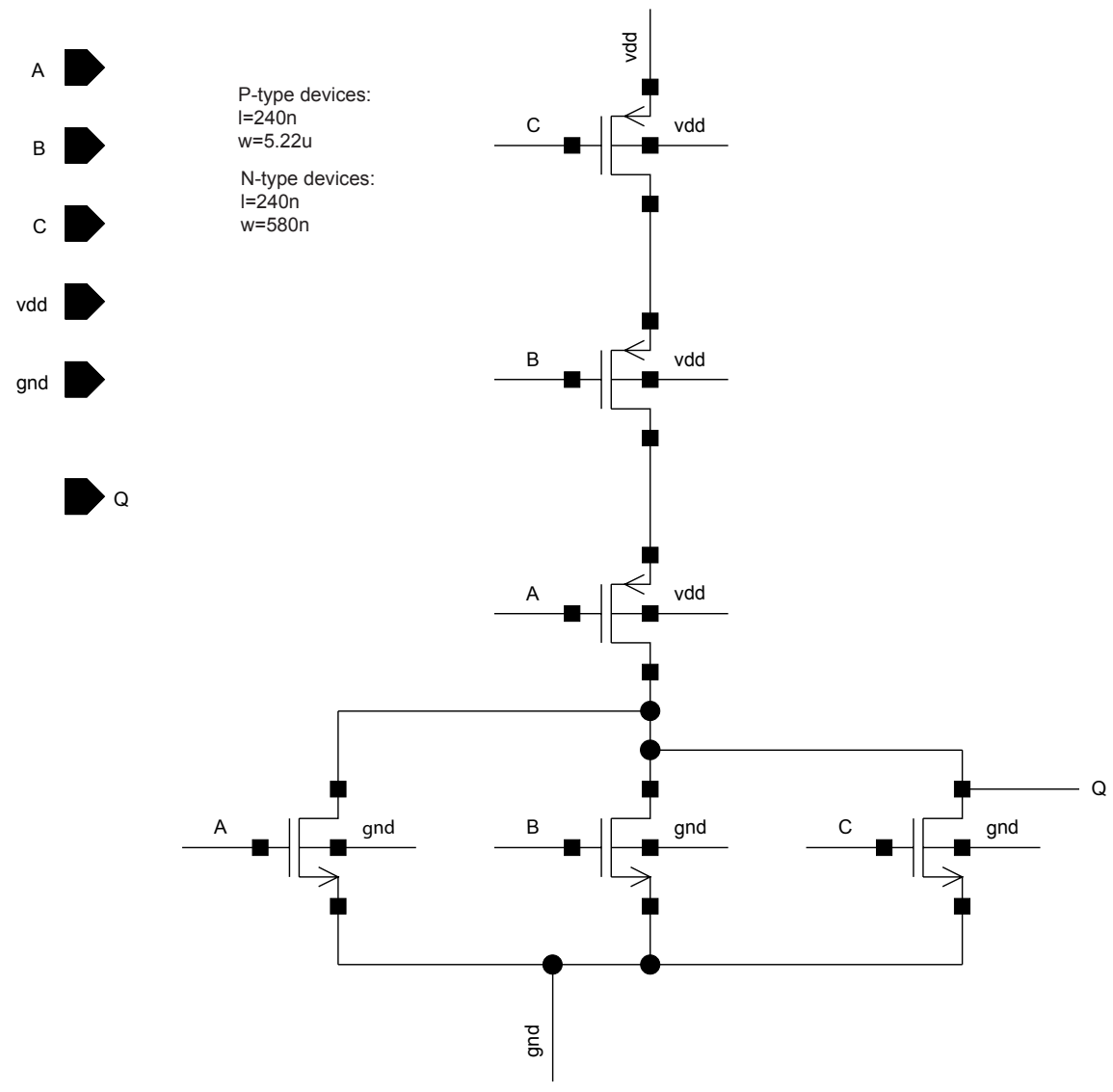

Figure A.4: Nor Gate 


\section{A.3 2's Complement Encoder}

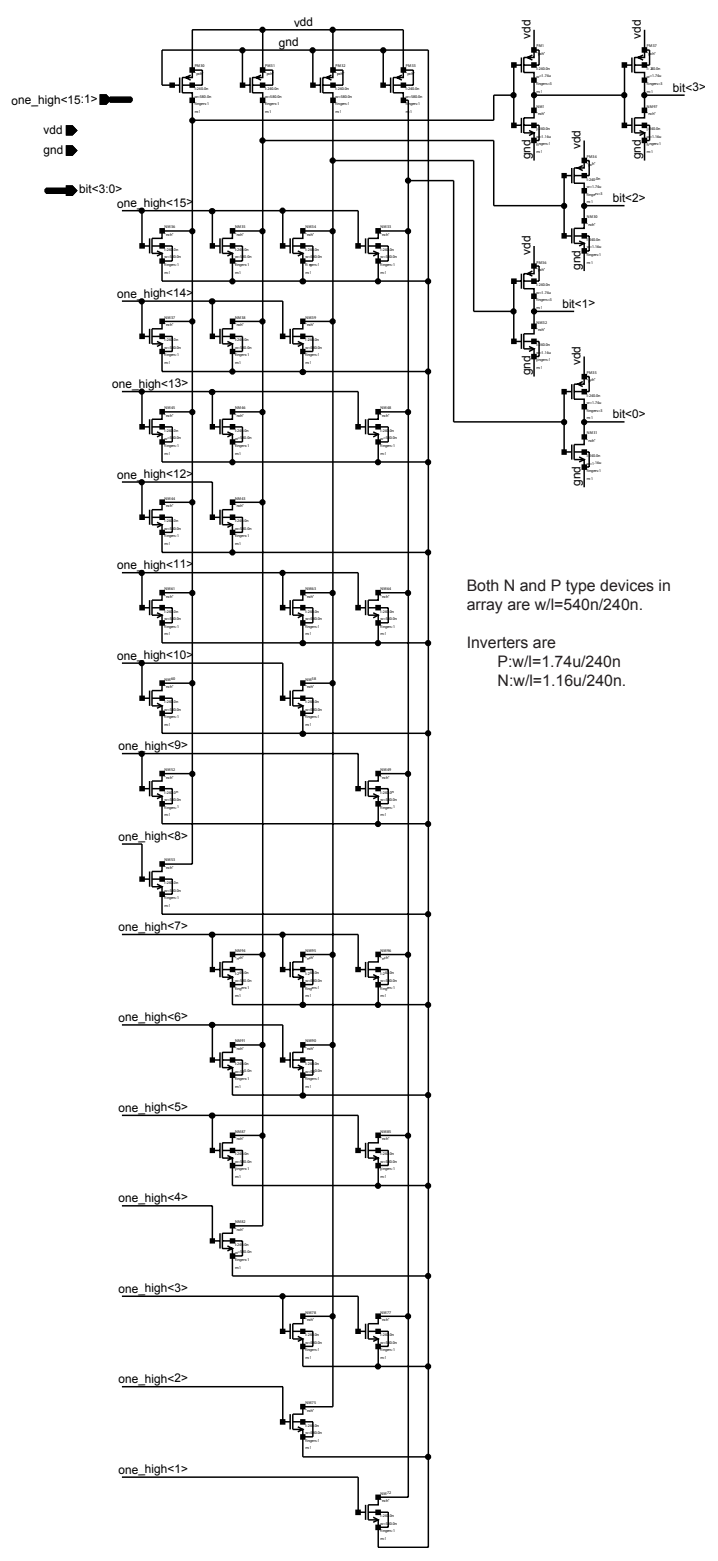

Figure A.5: 2's Complement Encoder 


\section{A.4 Unsigned Encoder}

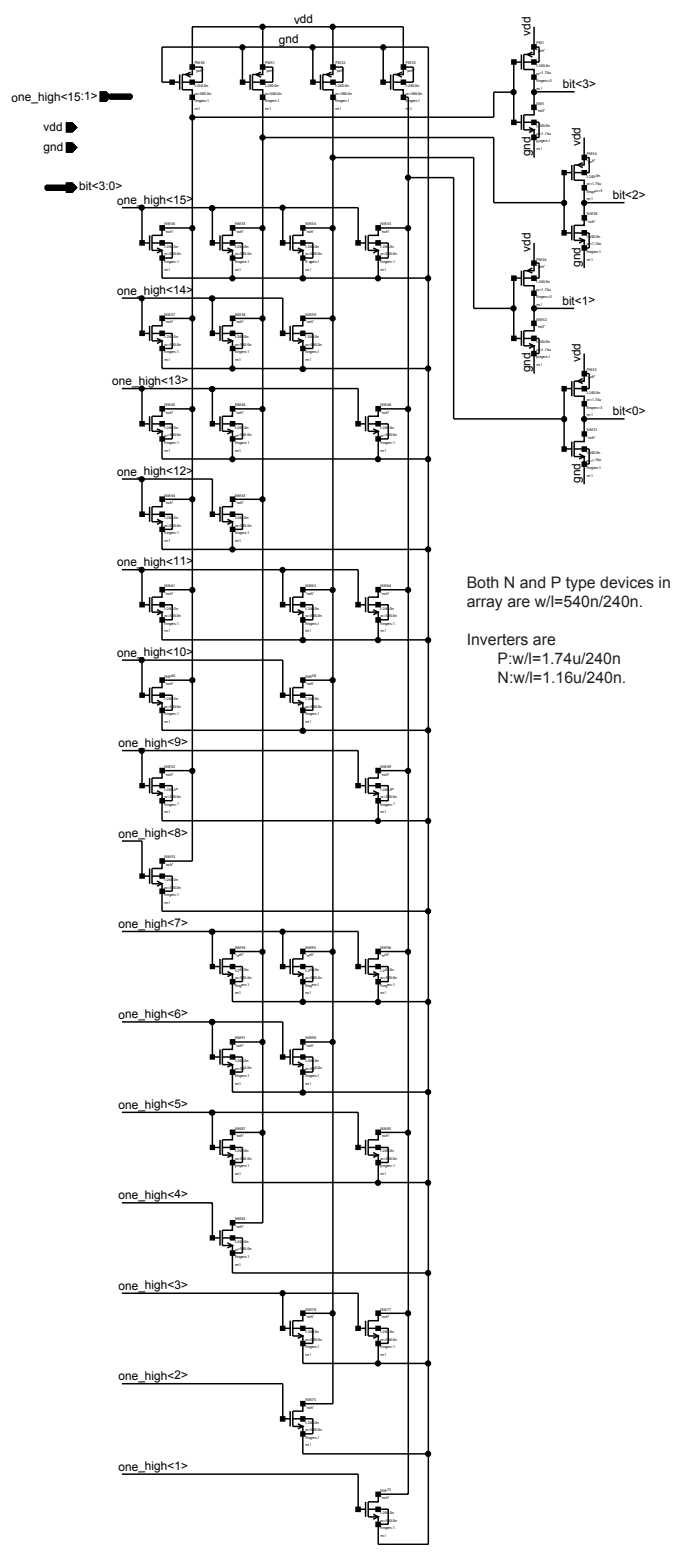

Figure A.6: Unsigned Encoder 


\section{A.5 ReQ}

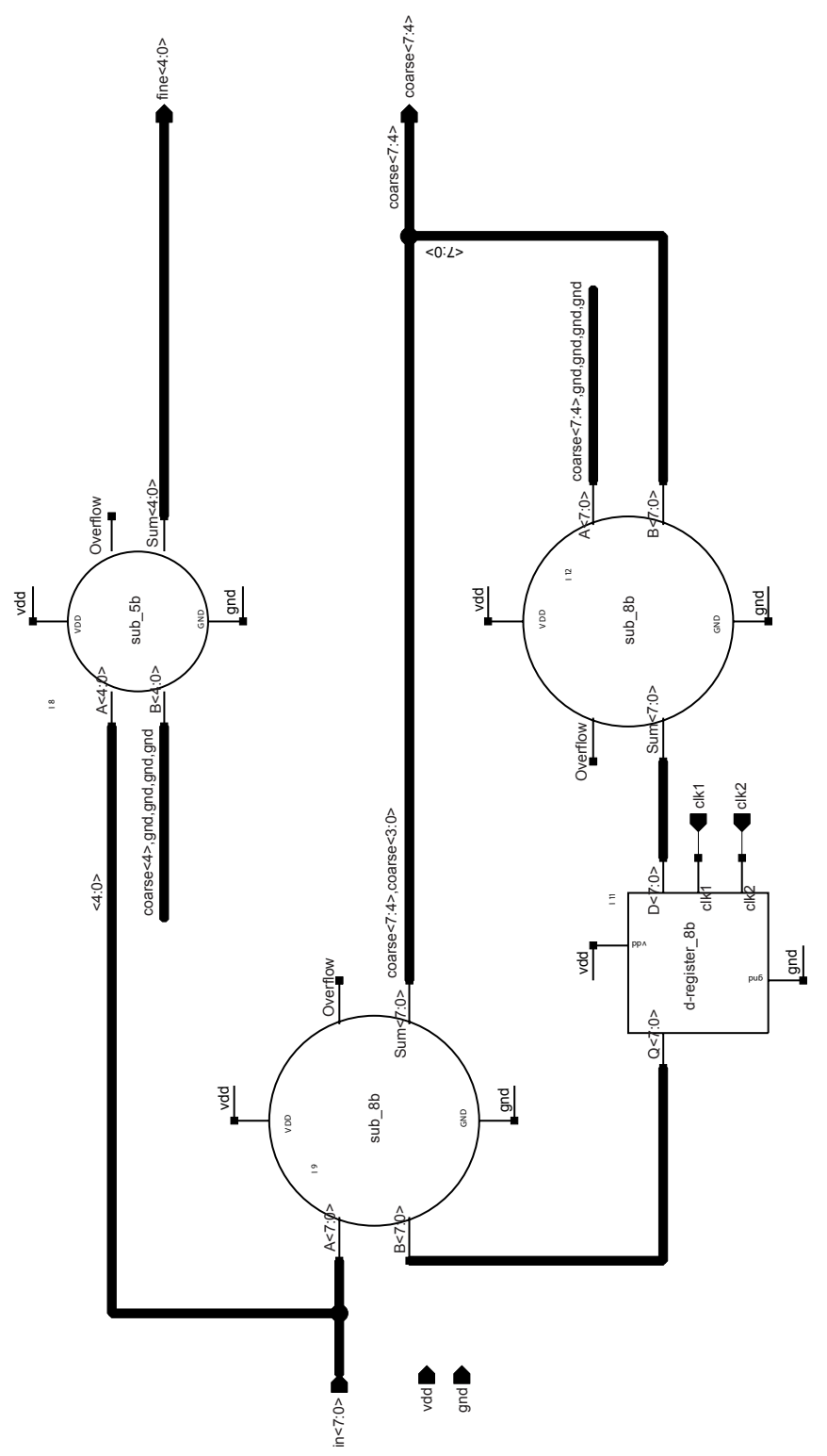

Figure A.7: Requantization Logic 


\section{A.5.1 8-bit Subtractor}

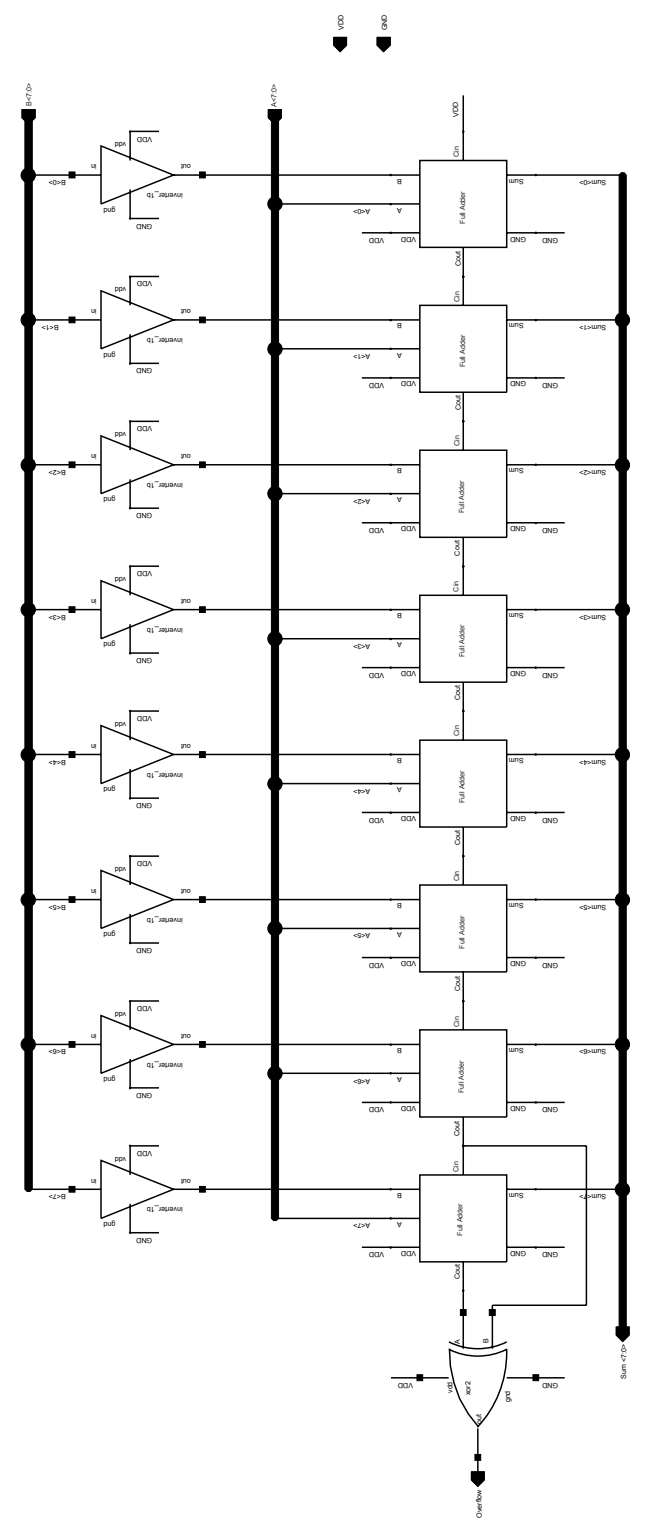

Figure A.8: 8-bit Subtractor 


\section{A.5.2 Full Adder}

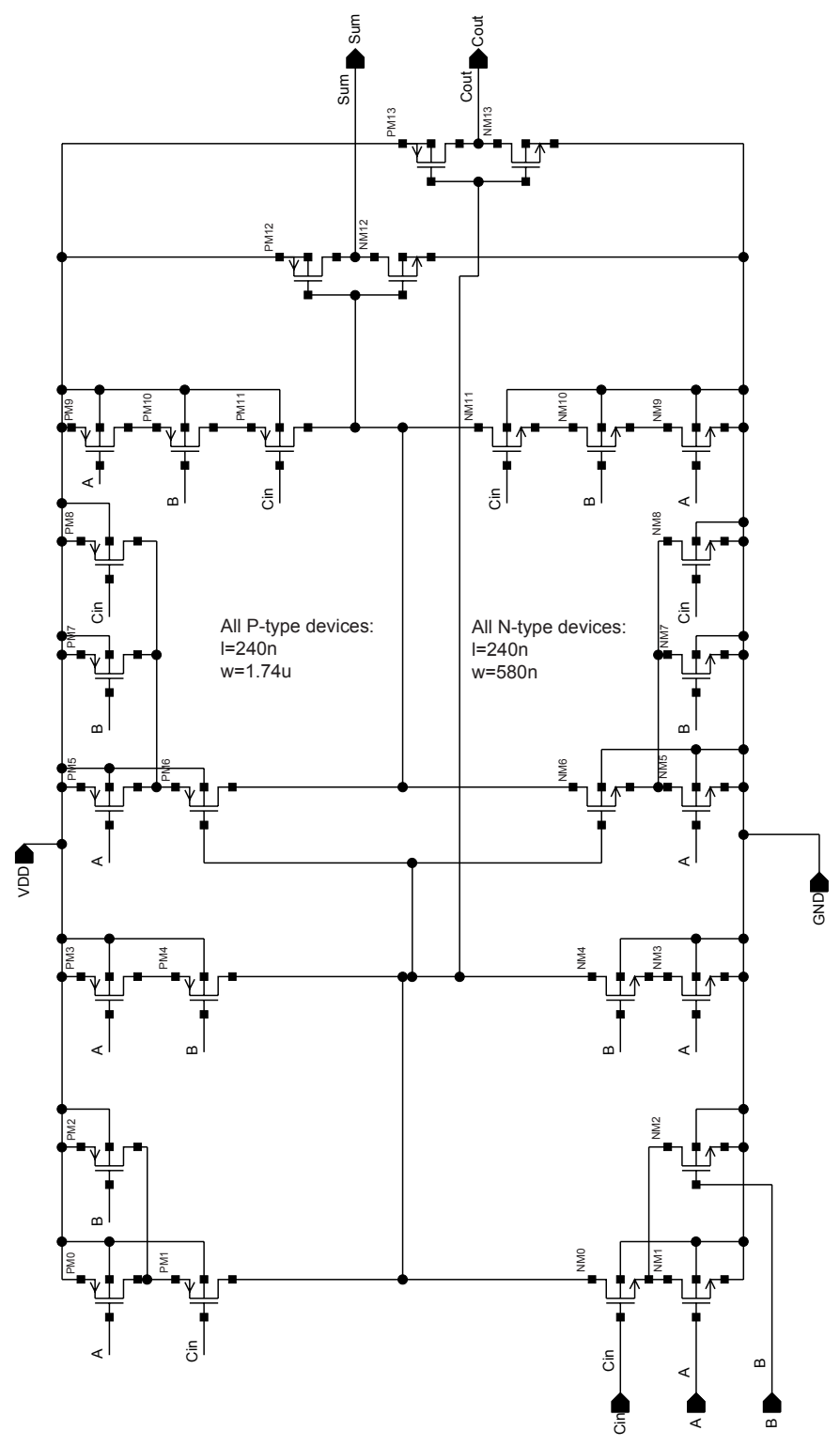

Figure A.9: Full Adder Design 


\section{A.5.3 5-bit Subtractor}

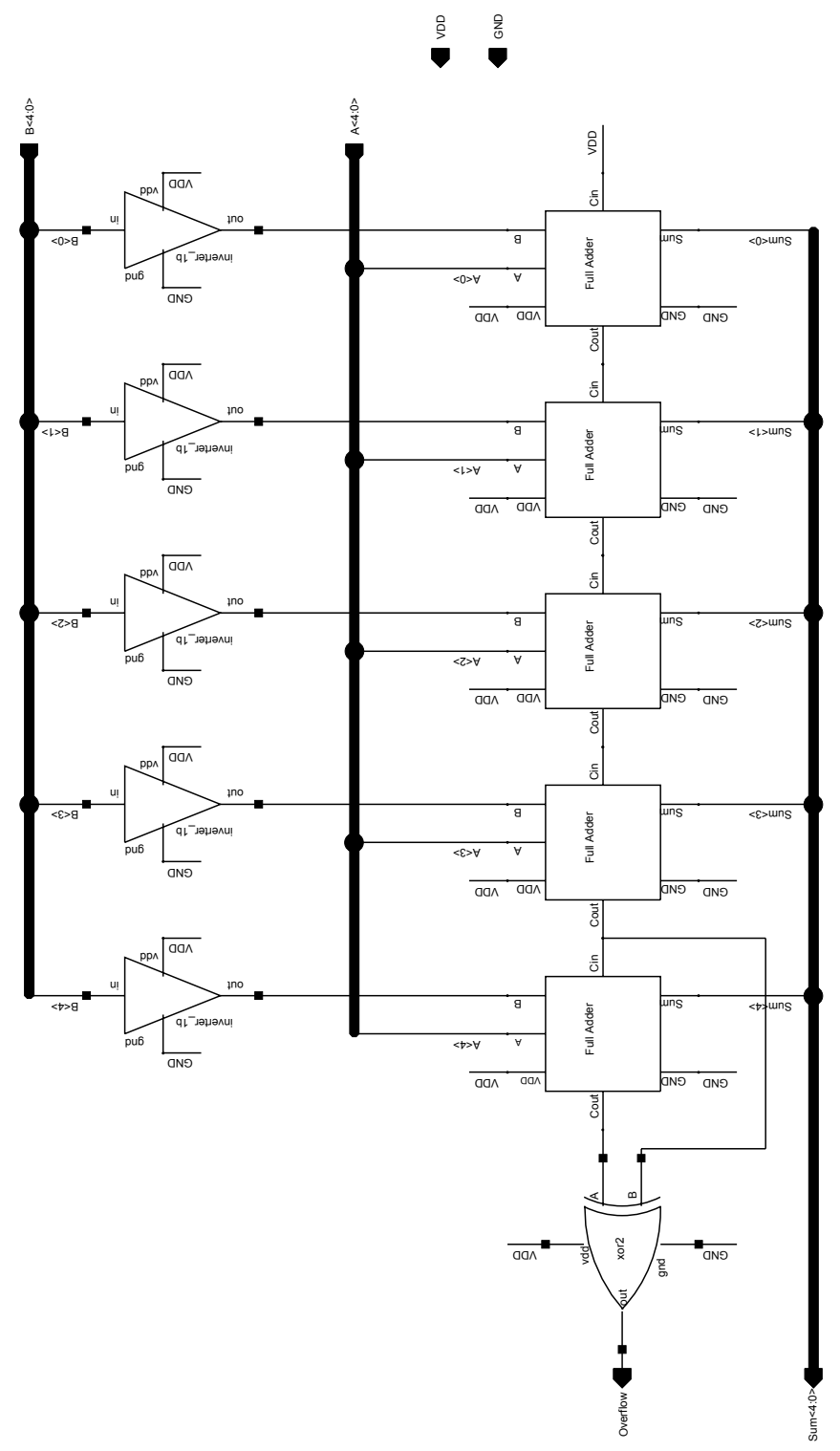

Figure A.10: 5-bit Subtractor 


\section{A.5.4 D-Register Bank}

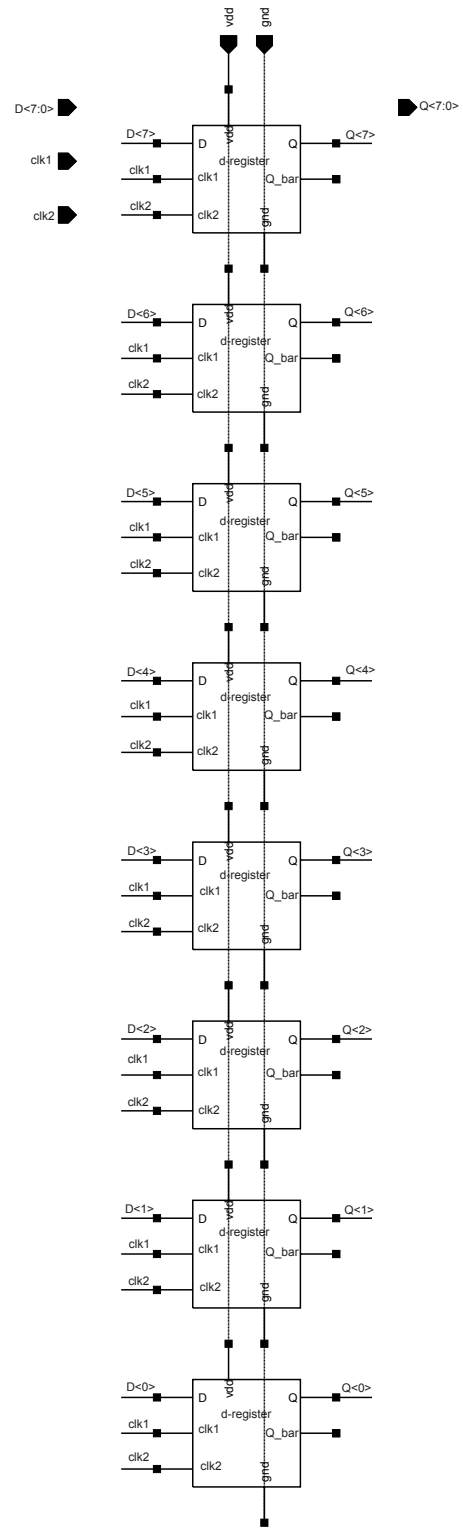

Figure A.11: D-Register Bank 


\section{A.5.5 D-Register}

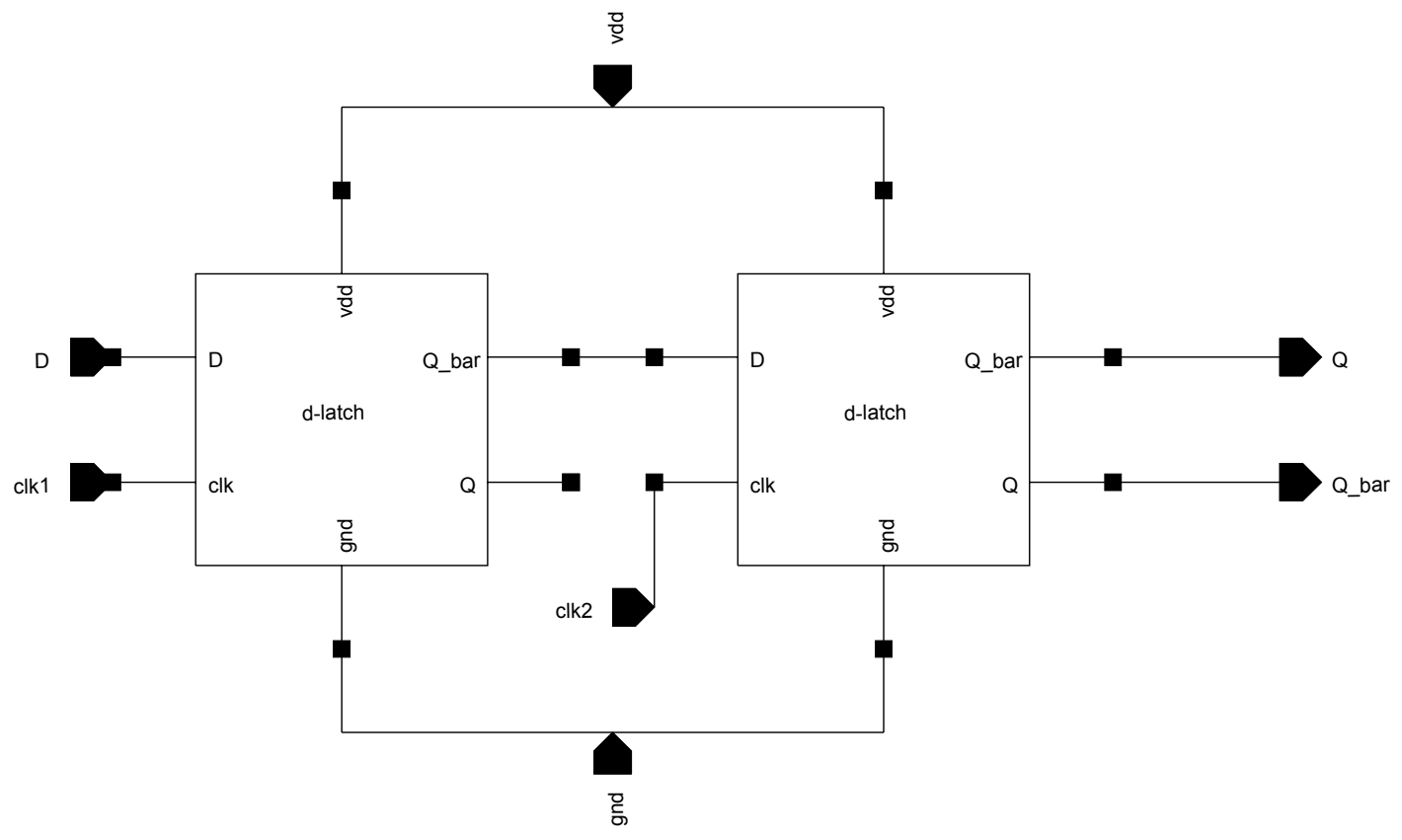

Figure A.12: D-Register 


\section{A.5.6 D-Latch}

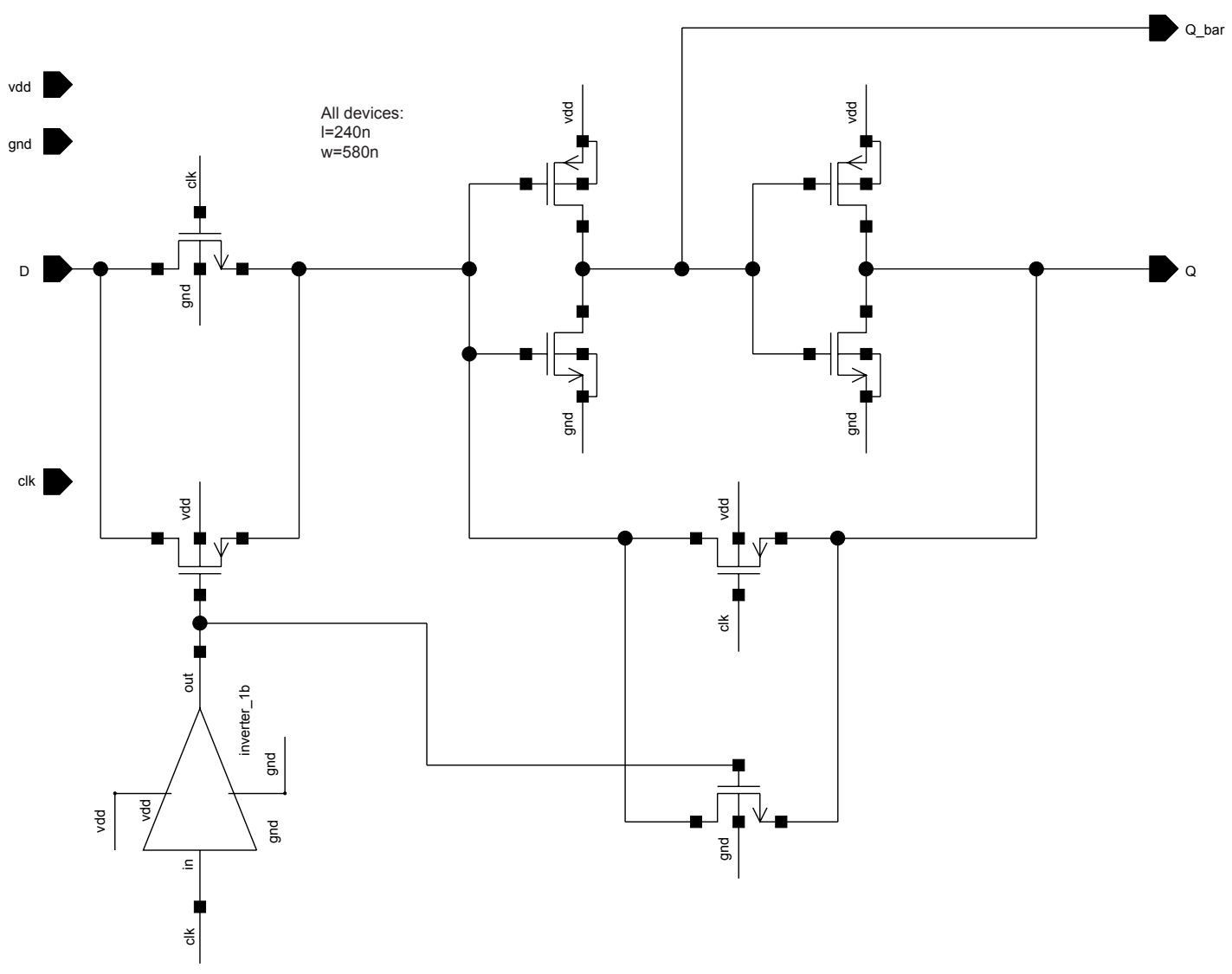

Figure A.13: D-Latch 


\section{A.6 4-bit Decoder Logic}

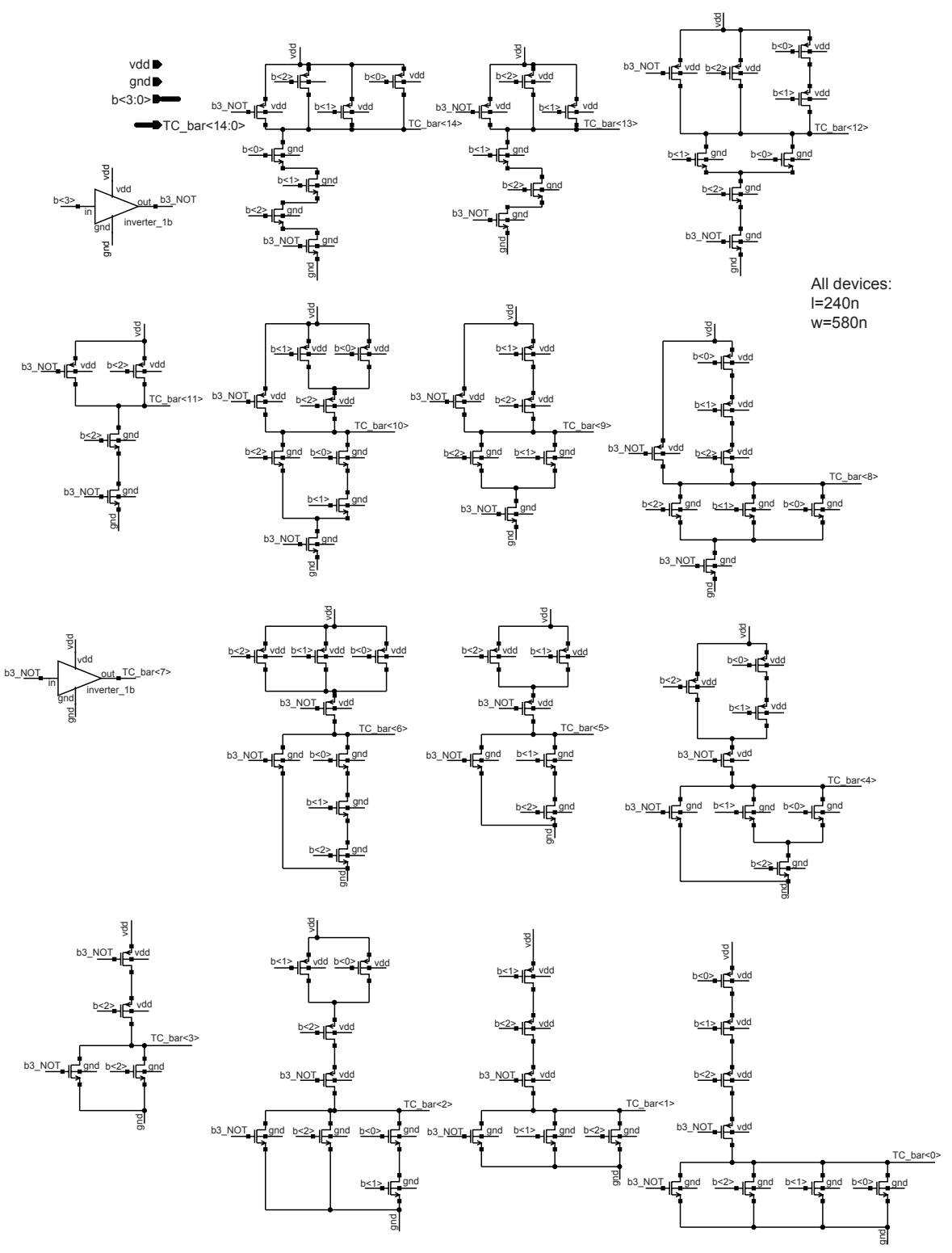

Figure A.14: 4-bit Binary-to-Thermometer Code Decoder 


\section{A.7 5-bit Decoder Logic}

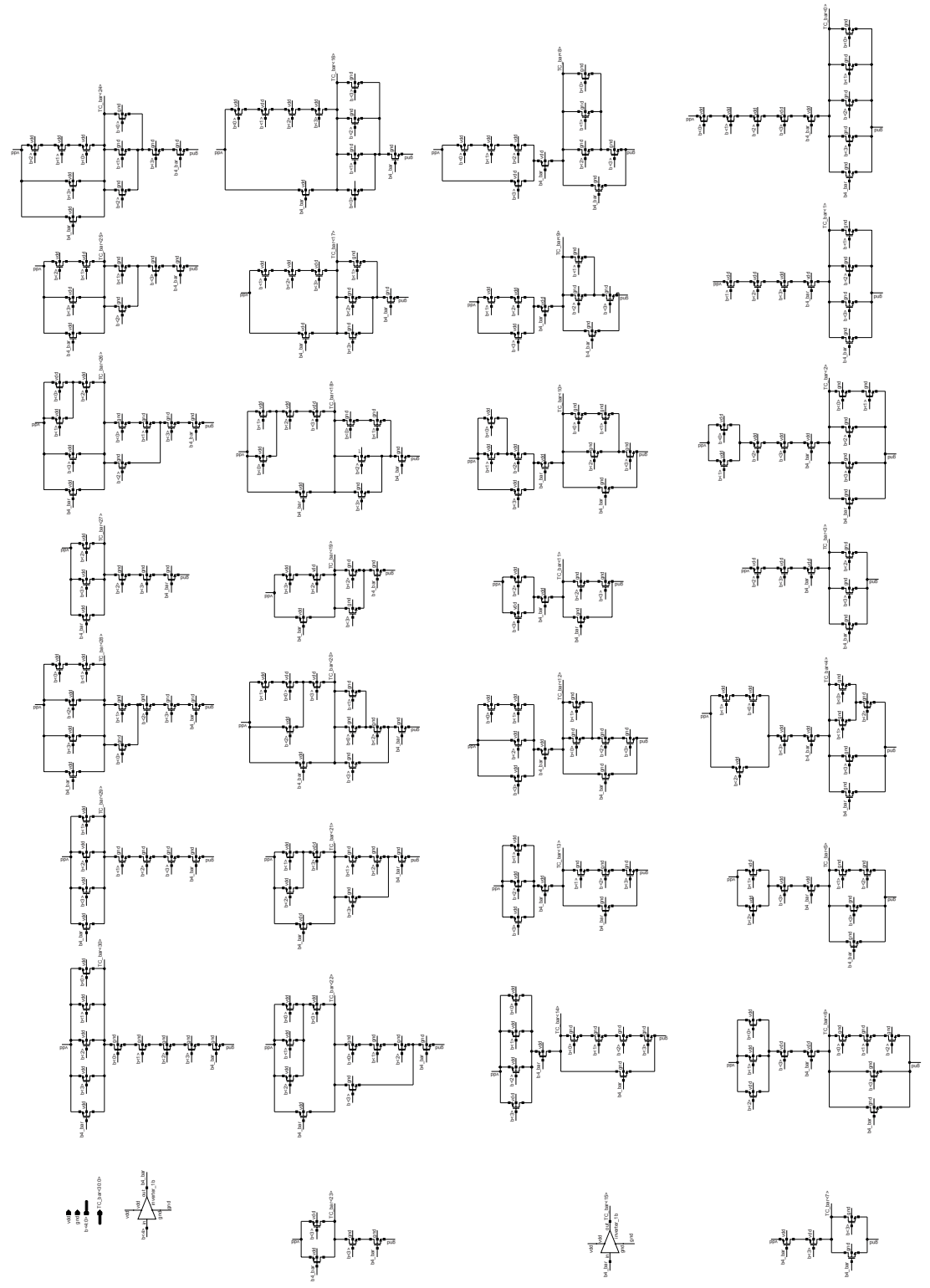

Figure A.15: 5-bit Binary-to-Thermometer Code Decoder 


\section{A.8 DEM Logic}

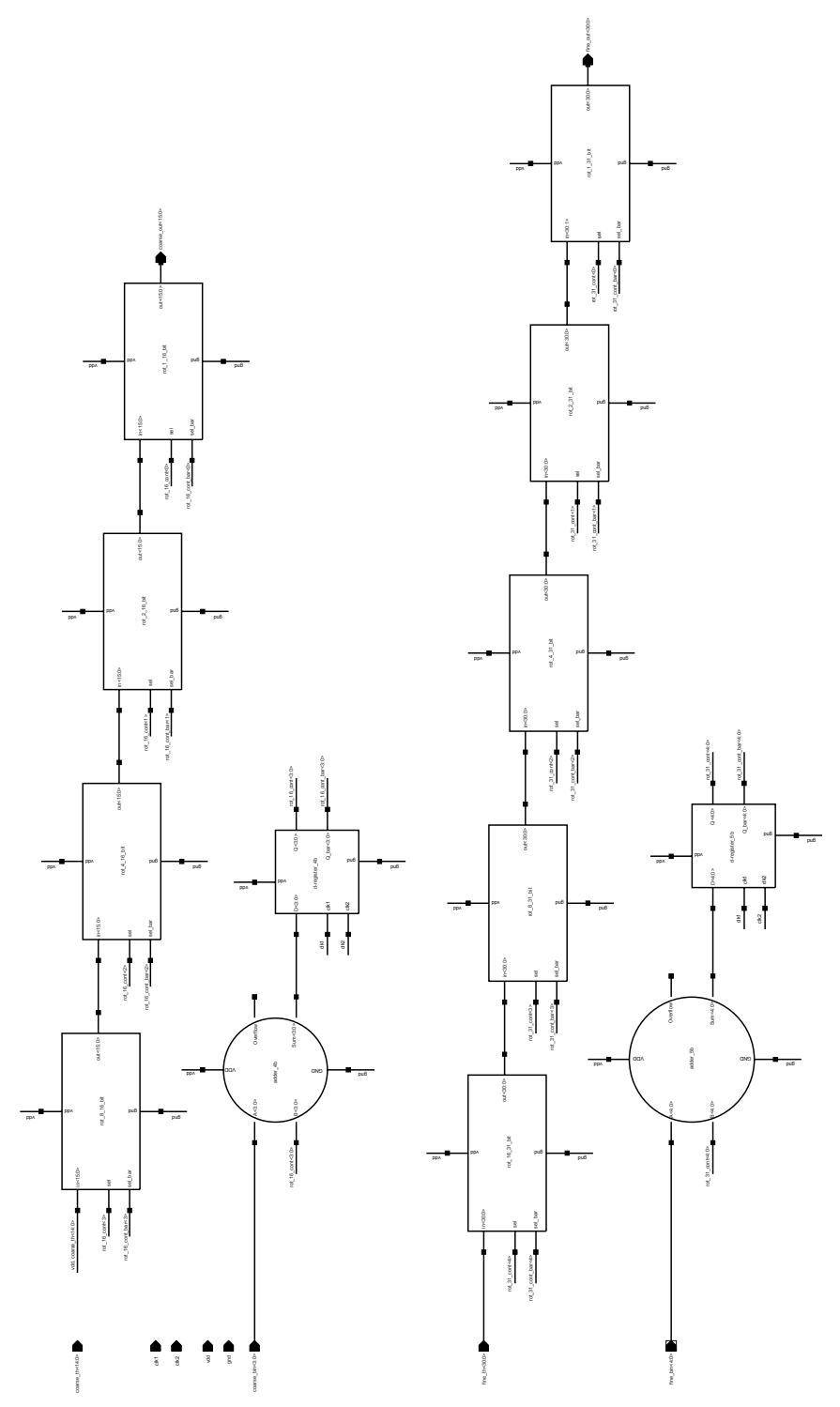

Figure A.16: Dynamic Element Matching Logic 


\section{A.8.1 16-line Rotate-1 Logic}
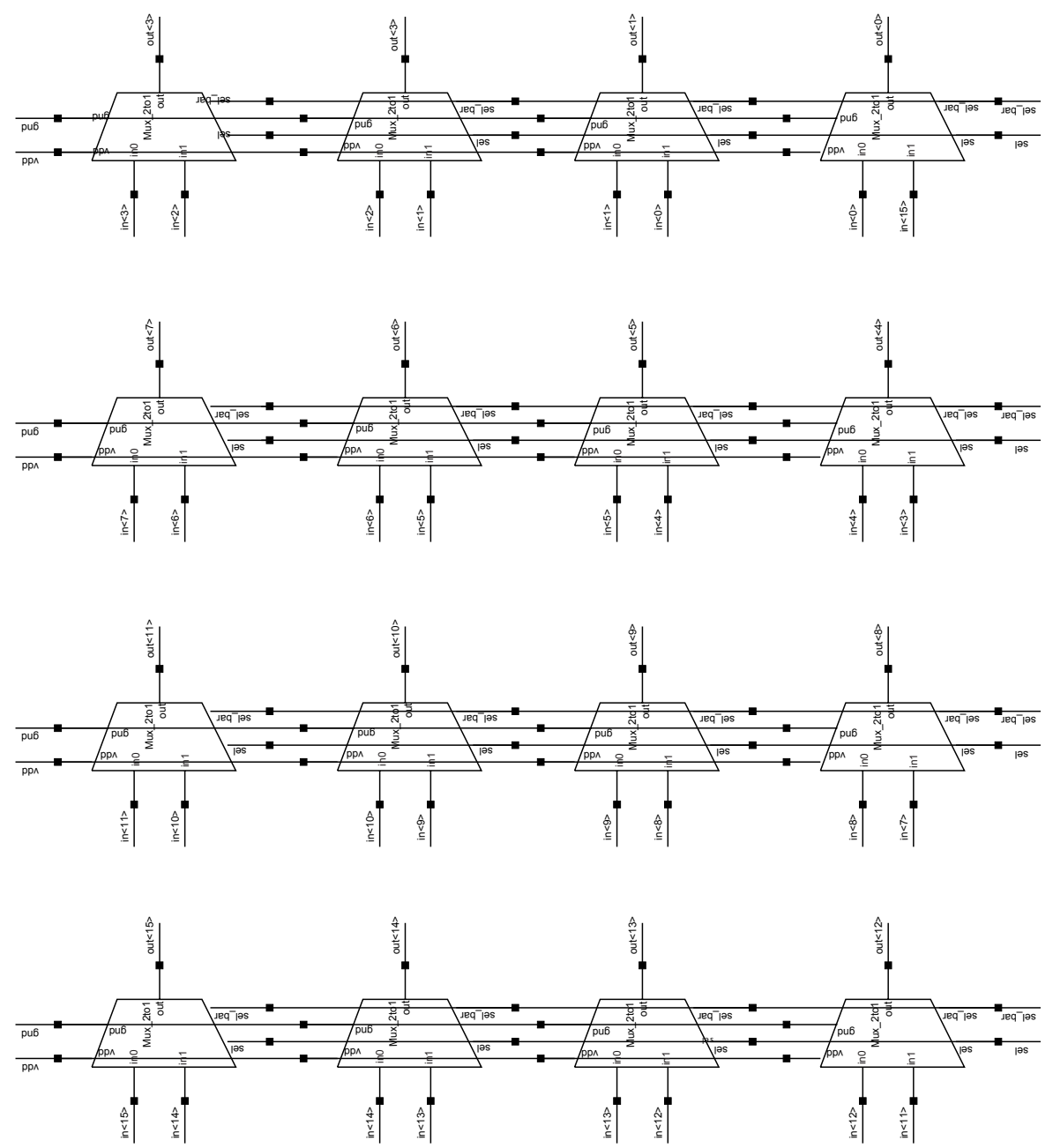

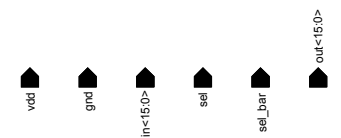

Figure A.17: 16-Input Rotate-1 


\section{A.8.2 31-line Rotate-1 Logic}
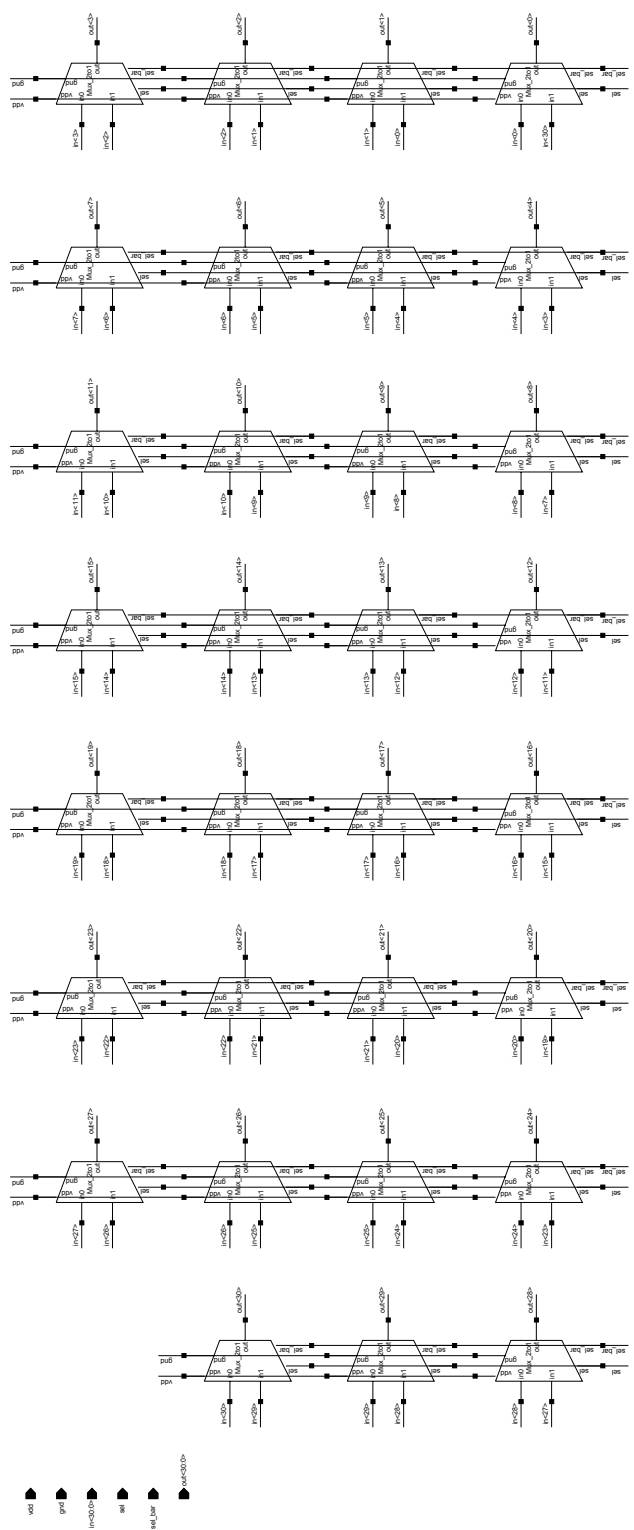

Figure A.18: 31-Input Rotate-1 


\section{A.8.3 2-to-1 Mux}
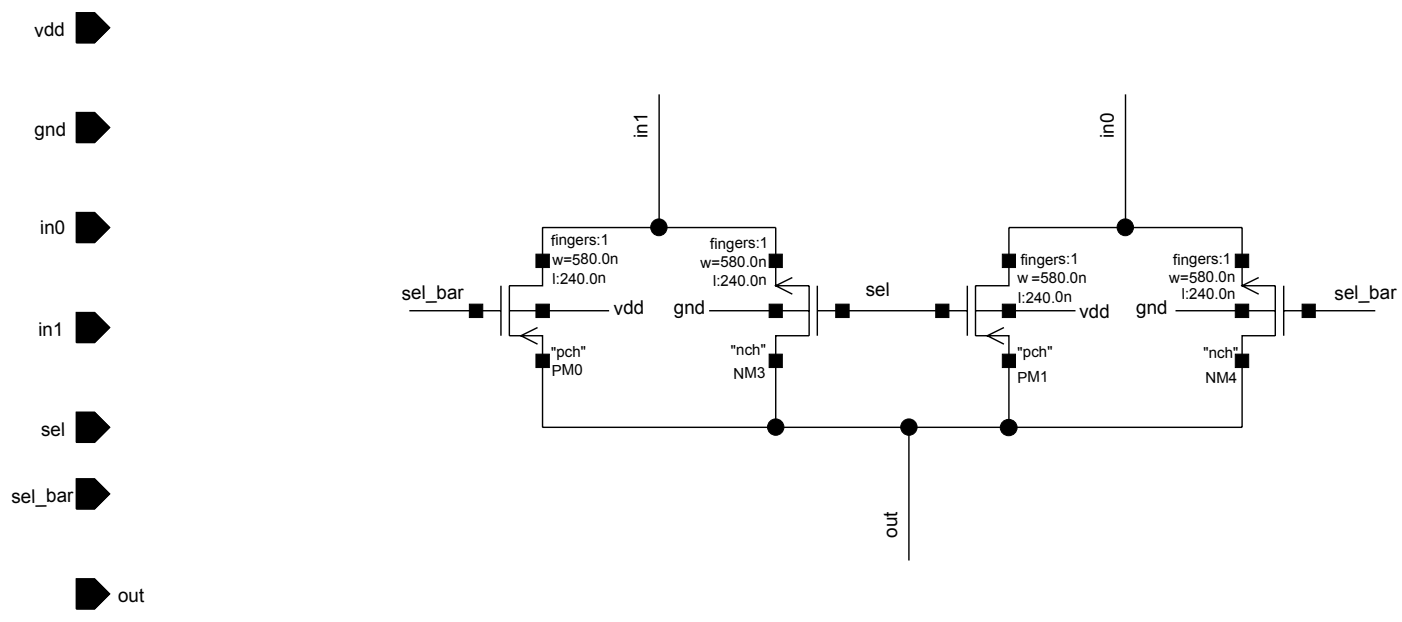

Figure A.19: 2-to-1 Mux 


\section{Appendix B}

\section{MATLAB Code}

\section{B.1 Reference Case Code}

function out $=$ second_order_reference (mismatch, iterate)

$\%$ 2nd order 8-bit sigma-delta model and noise analysis

$\%$ include DAC mismatch and regular, barrel-shifting DEM

$\%$ Craig Petrie 6/24/2002

$\%$ Modified by Brent Nordick

start_time = clock;

format long $g$

$\%$ model parameters

bin $=13 ; \quad \%$ number of sine wave cycles to capture (i.e., fft bin number)

ptspc $=1040 ; \%$ number of points per cycle to plot

amp $=0.8 ; \quad \%$ sine wave amplitude

ref $=1.0 ; \quad \%$ reference voltage (full-scale analog input, positive side)

levels $=256 ; \%$ number of internal quantizer levels

init $=4 ; \quad \%$ initialization points

OSR $=20 ; \quad \%$ oversampling ratio

dacmm $=0 ; \quad \%$ percent mismatch (in $\%$ ) of dac elements

sigbins $=1 ; \quad \%$ bins on either side of signal to lump with signal

NODEM $=0 ; \quad \%$ set to 1 to turn off DEM

loop_iterate $=1 ; \quad \%$ set up to run this many times for averaging

showme $=1 ; \quad \%$ variable to control display of values and plots

$\%$ startup as function necessary stuff

if nargin $>0$

dacmm = mismatch;

end

if nargin > 1

loop_iterate = iterate;

end

if nargout $>0$

showme $=0$;

end

$\%$ Initial calculations

pts = bin*ptspc;

norm_factor $=$ ptspc $/(2 * 0 \mathrm{SR})$; 
$\%$ calculate parameters for internal adc function

$\operatorname{lm} 1$ = levels - 1 ;

$\mathrm{m}=\operatorname{lm} 1 / 2 /$ ref; $\quad \%$ slope

$\mathrm{b}=\operatorname{lm} 1 / 2 ;$

$\%$ set up loop for averaging purposes

for lop $=1$ :loop_iterate

$\mathrm{x}=\operatorname{amp} * \sin (2 * \mathrm{pi} * \mathrm{bin} *[0: \mathrm{pts}-1+\mathrm{init}] / \mathrm{pts}) ; \quad \%$ exactly 'bin' cycles of

$\%$ 'pts'-point sine wave $\mathrm{x}=\mathrm{A} * \sin (2 * \mathrm{pi} * \mathrm{w} * \mathrm{t})$

$\% \mathrm{x}=-1 *$ ones $(1$, length $(\mathrm{x})) ;$

$\% D C$ input for testing purposes

elm1 = getdac (levels, ref, dacmm); $\quad \%$ retrieve dac element values for dac \#1

elm2 = getdac (levels, ref, 0$)$;

$\%$ retrieve dac element values for dac \#2

$\%$ main delta-sigma loop

$\mathrm{u}=0 ; \mathrm{v}=0$;

$\%$ initialize integrator outputs

demptr $=0$;

$\%$ initialize DEM pointer

$\mathrm{y}=\operatorname{zeros}(1, \mathrm{pts}+\mathrm{init}) ; \quad \%$ allocate and initialize output vector

oneelm $=\operatorname{zeros}(1, \mathrm{pts}+$ init $) ; \quad \%$ examine frequency content of a single

for $i=1: p t s+i n i t$

$\%$ element signal

$[\mathrm{z}, \mathrm{y}(\mathrm{i})]=\operatorname{idealq}(\mathrm{v}, \mathrm{m}, \mathrm{b}, \operatorname{lm} 1)$;

$\%$ quantize using second

$\%$ integrator output before 2 nd

if (NODEM)

$\%$ integration

demptr $=0$;

end $\quad \%$ remove DEM pointer memory to turn off DEM

$[$ dacvec, demptr $]=\operatorname{dem}(z, l m 1$, demptr) $; \quad \%$ call DEM (Dynamic Element

$\%$ Matching) algorithm

$\operatorname{oneelm}(i)=\operatorname{dacvec}(1)$;

$\%$ look at first element

$\mathrm{v}=\mathrm{v}+2 *(\mathrm{u}-\operatorname{sum}($ dacvec.*elm 2$)) ; \quad \%$ 2nd integration, use 1st

$\%$ integrator output before 1st integration

$u=u+0.5 *(x(i)-\operatorname{sum}($ dacvec. $*$ elm 1$)) ; \%$ 1st integration

end

$\%$ plot waveforms, spectrum

$\mathrm{x}=\mathrm{x}($ init $+1: \mathrm{pts}+$ init $)$;

$\%$ grab last part of data - ignore init portion

$y=y($ init $+1: p t s+i n i t)$;

$\%$ grab last part of data - ignore init portion

ptsv $=1:$ pts

yspec $=\operatorname{mypsd}(y)$;

$\%$ calculate snr

sigtonoise $=$ mysnr $(y s p e c$, bin, OSR);

sig_to_noise (lop) = sigtonoise; $\%$ vector of values to average

$\%$ compare with ideal snr of 2 nd-order modulator

sigi $=\operatorname{amp} /$ sqrt $(2) ; \quad \%$ rms voltage of sine wave

noisei $=2 *$ ref $/$ levels $/ \operatorname{sqrt}(12) *(\mathrm{pi})^{\wedge} 2 / \operatorname{sqrt}(5) /(\text { OSR })^{\wedge} 2.5$;

$\%$ theoretical rms quantization noise voltage

sigtonoisei $=20 * \log 10($ sigi $/$ noisei $) ;$

$\%$ calculate sfdr

sigv $=\operatorname{yspec}(\max ([1,($ bin-sigbins $)]):$ bin+sigbins $) ; \%$ signal plus spectral bleed noisev $=[y \operatorname{spec}(1:$ bin-sigbins-1) yspec $($ bin+sigbins+1:pts/2/OSR $)] ;$ vector of

$\%$ bins used to calculate noise 


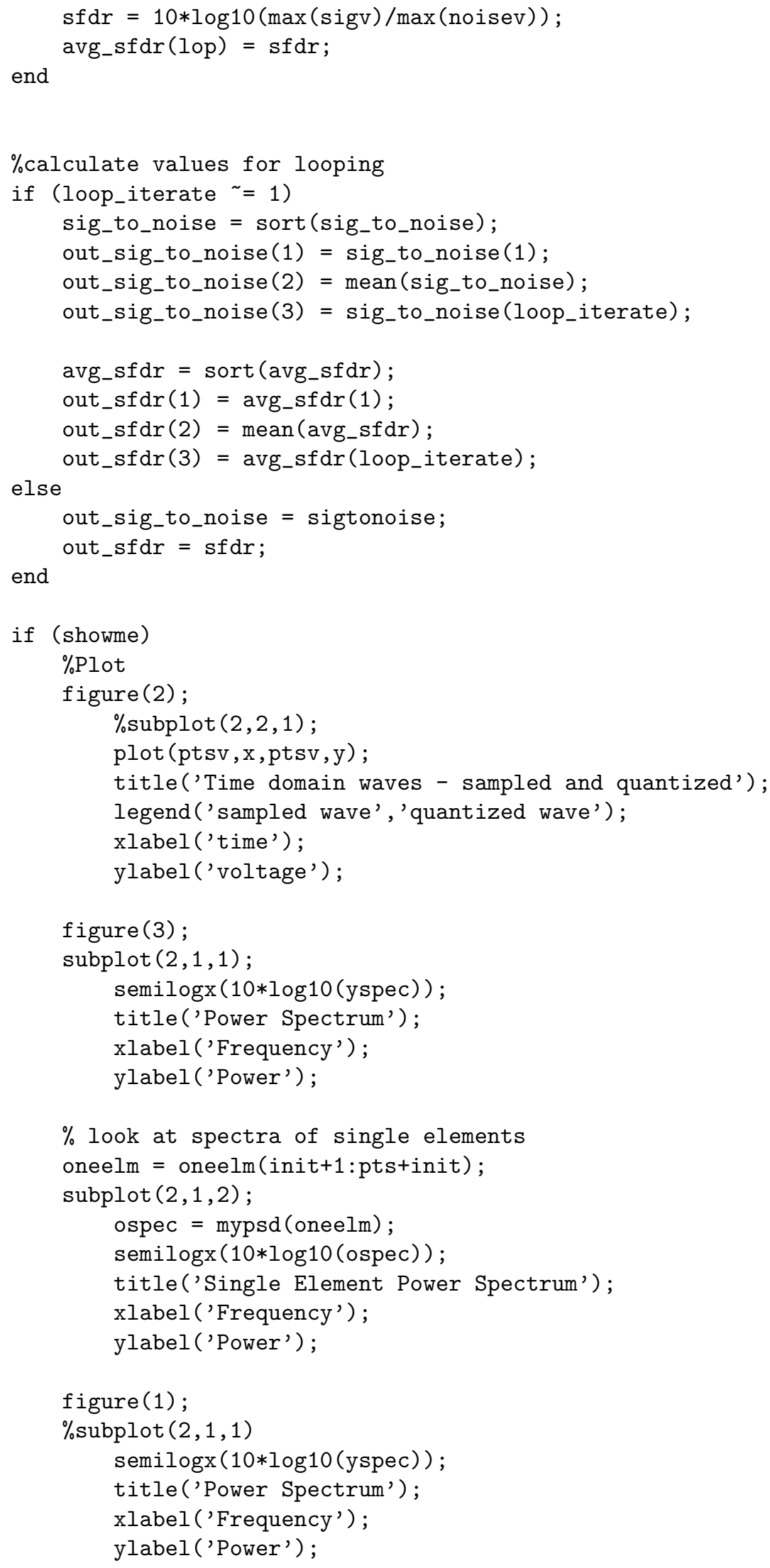


$\%$ display avg/max/min on looping, or just the output when not

sigtonoisei

out_sig_to_noise

out_sfdr

else

$\%$ output of the function

out = out_sig_to_noise;

end

elapsed_time $=$ etime (clock, start_time)

function elmvec $=\operatorname{getdac}($ levs, fs, mm)

$\%$ calculate random element values for the dac; each element either added or

$\%$ subtracted

$\%$ levs output levels generated by the dac; there are (levs-1) elements

$\%$ fs full scale output value, positive side

$\% \quad m m \quad$ desired dac element mismatch, in percent

$\% \quad$ elmvec output vector containing (levs-1) dac element values

nom $=f s /($ levs -1$)$

$\%$ ideal dac element value (mean $\%$ of output array)

elmvec $=\operatorname{nom} *(1+0.01 * m m * r a n d n(1$, levs-1) $) ; \quad \% 1-b y-($ levs -1$)$ array of random $\%$ element values

function [intout, dubout] = idealq(in, $\mathrm{m}, \mathrm{b}, \operatorname{maxin}$ )

$\%$ ideal quantization using pre-calculate slope and intercept values

$\%$ in value to be quantized

$\% \quad \mathrm{~m} \quad$ input multiplier before rounding

$\% \quad b \quad$ input offset before rounding

$\%$ maxin output code forced to be in range [0,maxin]

$\%$ codout output integer quantized value

$\%$ dubout output double quantized value (with same input range as 'in')

intout $=\operatorname{round}(\mathrm{m} *$ in $+\mathrm{b})$;

intout $=\max ([$ intout, 0$])$;

intout $=\min ([$ intout, $\operatorname{maxin}])$;

dubout $=($ intout $-\mathrm{b}) / \mathrm{m}$;

function [vecout,ptrout] = dem(code, $\operatorname{lm} 1$, ptrin)

$\%$ perform barrel-shifing dem algorithm

$\%$ code input integer code

$\% \quad \operatorname{lm} 1 \quad$ (\# dac output levels) - 1; number of dac elements

$\%$ ptrin position where elements start getting used this time, in range

$\%$ [0, levs -1$]$

$\%$ vecout boolean output vector (+1 or -1) corresp to 'on' elements in the DAC

$\%$ ptrout position where elements start getting used next time, in range

$\% \quad[0$, levs -1$]$

ptrout $=\bmod ($ ptrin+code, $\operatorname{lm} 1)$;

if $($ (ptrout $>$ ptrin) | (code $==0)$ )

vecout $=[\operatorname{repmat}(-1,1, \operatorname{ptrin}), \operatorname{repmat}(1,1, \operatorname{code}), \operatorname{repmat}(-1,1, \operatorname{lm} 1-\operatorname{code}-\operatorname{ptrin})]$;

else $\%$ (ptrout < ptrin) | (code == lm1)

vecout $=$

$[\operatorname{repmat}(1,1, \operatorname{code}+\operatorname{ptrin}-\operatorname{lm} 1), \operatorname{repmat}(-1,1, \operatorname{lm} 1$-code $), \operatorname{repmat}(1,1, \operatorname{lm} 1-\operatorname{ptrin})]$;

end 


\section{B.2 Calibration Code}

function out $=$ second_order_calibrate(mm_fine, iterate)

$\%$ 2nd order sigma-delta model and noise analysis

$\%$ include DAC mismatch and course/fine DEM

$\%$ Craig Petrie 6/24/2002

$\%$ Brent Nordick 12-10-03

start_time = clock;

format long $g$

$\%$ model parameters

bin $=13 ; \quad \%$ number of sine wave cycles to capture (i.e., fft bin number)

ptspc $=1040 ; \%$ number of points per cycle

amp $=0.8 ; \quad \%$ sine wave amplitude

ref $=1.0 ; \quad \%$ reference voltage (full-scale analog input, positive side)

clevs $=16 ; \quad \%$ number of course levels (output codes) in two-step internal

$\%$ quantizer

flevs $=16 ; \quad \%$ number of fine levels in internal quantizer

init $=4 ; \quad \%$ initialization points

OSR $=20 ; \quad \%$ oversampling ratio

dacmmc $=0 ; \quad \%$ percent mismatch (in $\%$ ) of course dac elements

dacmmf $=0 ; \quad \%$ percent mismatch (in $\%$ ) of fine dac elements

sigbins $=1 ; \quad \%$ bins on either side of signal to lump with signal

NODEM $=0 ; \quad \%$ set to 1 to turn off DEM

loop_iterate $=1 ; \%$ Make it loop to get average StoN

showme $=1$; $\quad \%$ variale to control display of values and plots

$\%$ startup as function necessary stuff

if nargin $>0$

dacmmf $=$ mm_fine;

end

if nargin > 1

loop_iterate = iterate;

end

if nargout >0

showme $=0$;

end

$\%$ Initial calculations

pts $=$ bin*ptspc;

norm_factor $=$ ptspc $/(2 * 0 S R)$;

$\%$ calculate parameters for internal two-step adc function

$\%$ for fine quantization, input signal is in the range [-ref/clevs, +ref/clevs]

olevs = clevs*flevs; $\%$ number of overall levels in internal quantizer

olm1 = olevs - 1;

$\operatorname{clm} 1=$ clevs -1 ;

$\mathrm{flm} 1=\mathrm{flevs}-1$;

$\mathrm{mc}=\mathrm{olm} 1 / 2 / \mathrm{flevs} / \mathrm{ref} ; \%$ slope for course quantization

$\mathrm{bc}=\mathrm{clm} 1 / 2 ; \quad \%$ offset for course quantization

$\mathrm{mf}=\mathrm{olm} 1 / 2 /$ ref; $\quad \%$ slope for fine quantization

$\mathrm{bf}=\mathrm{flm} 1 / 2 ; \quad \%$ offset for fine quantization 


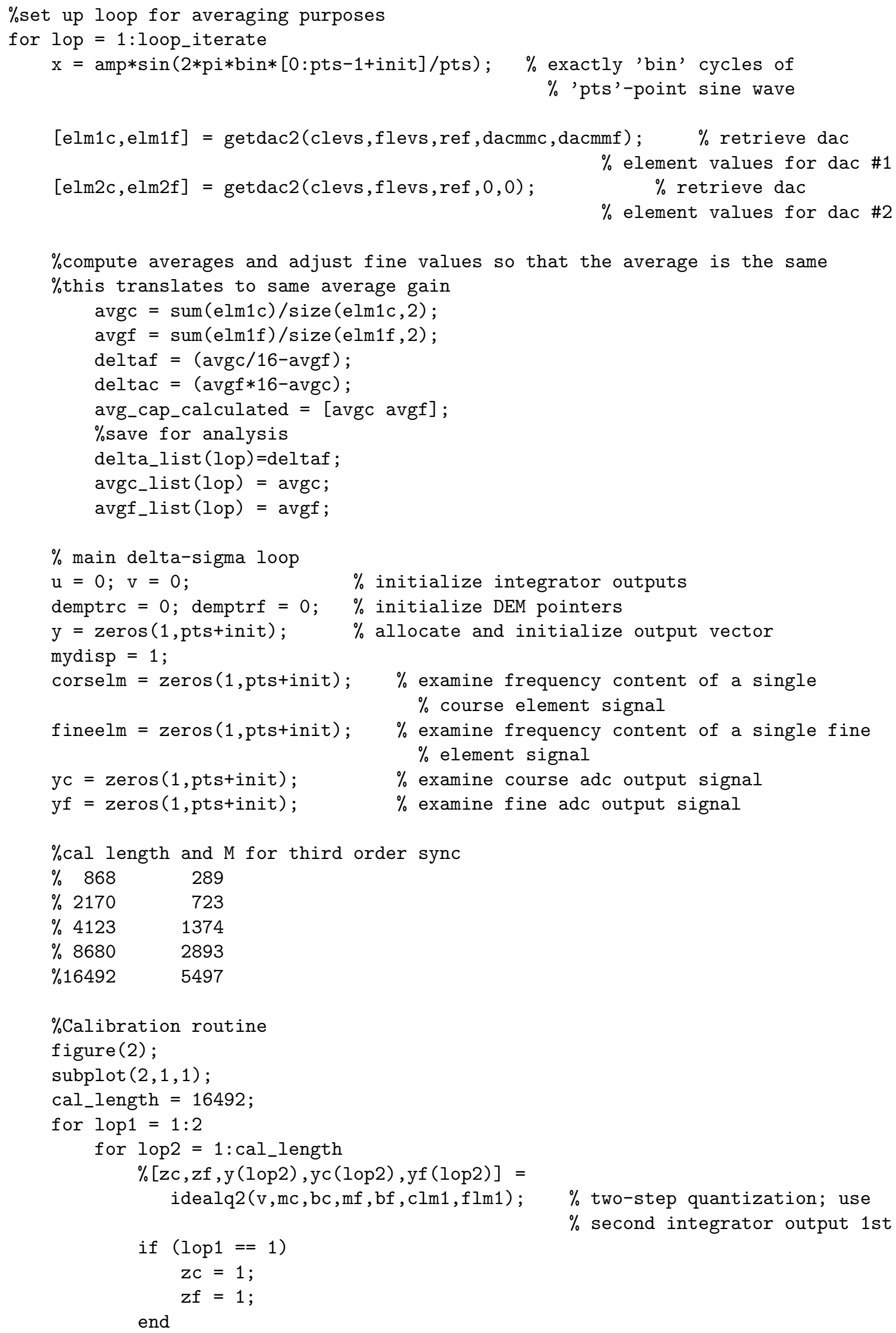




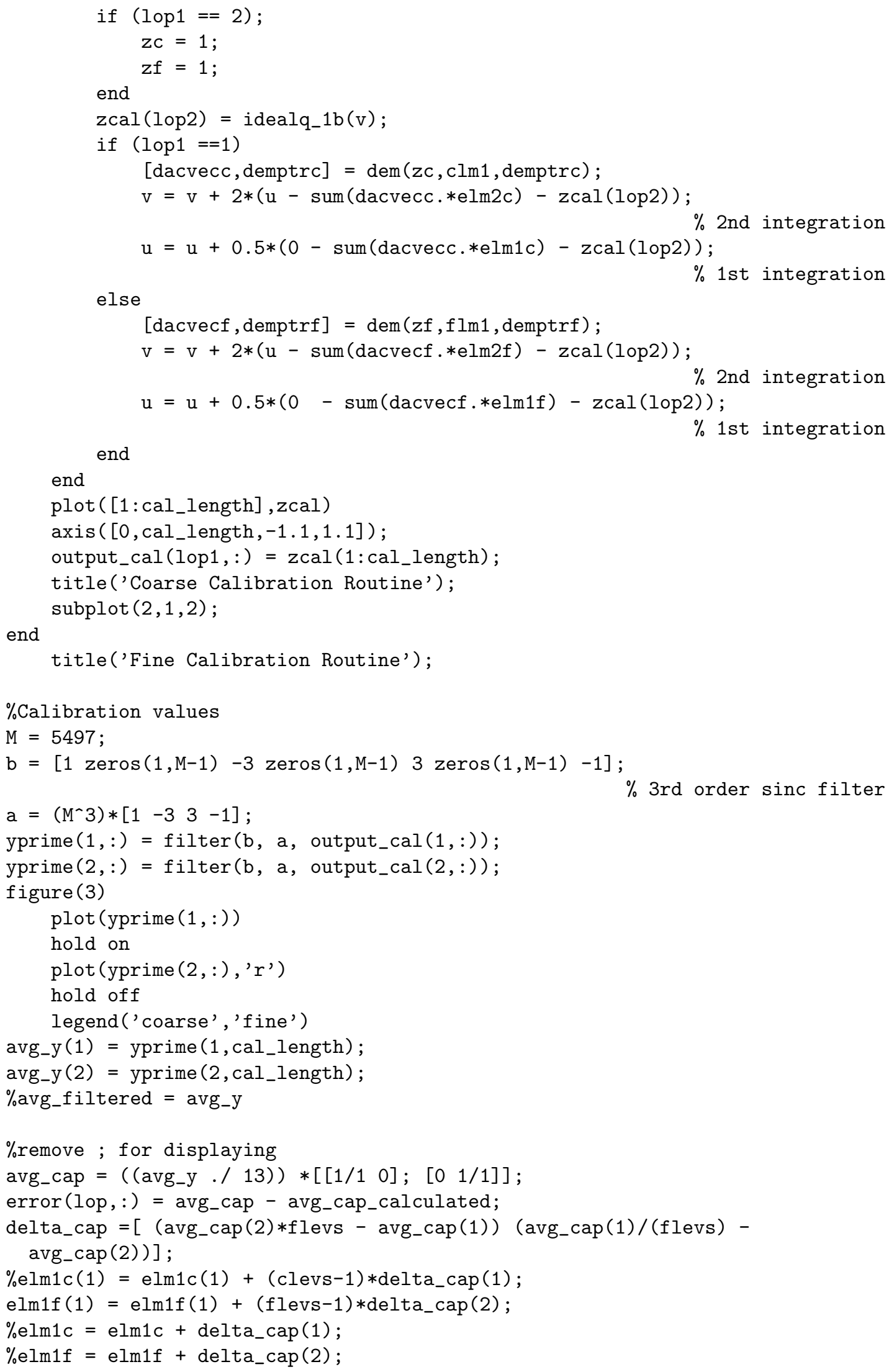




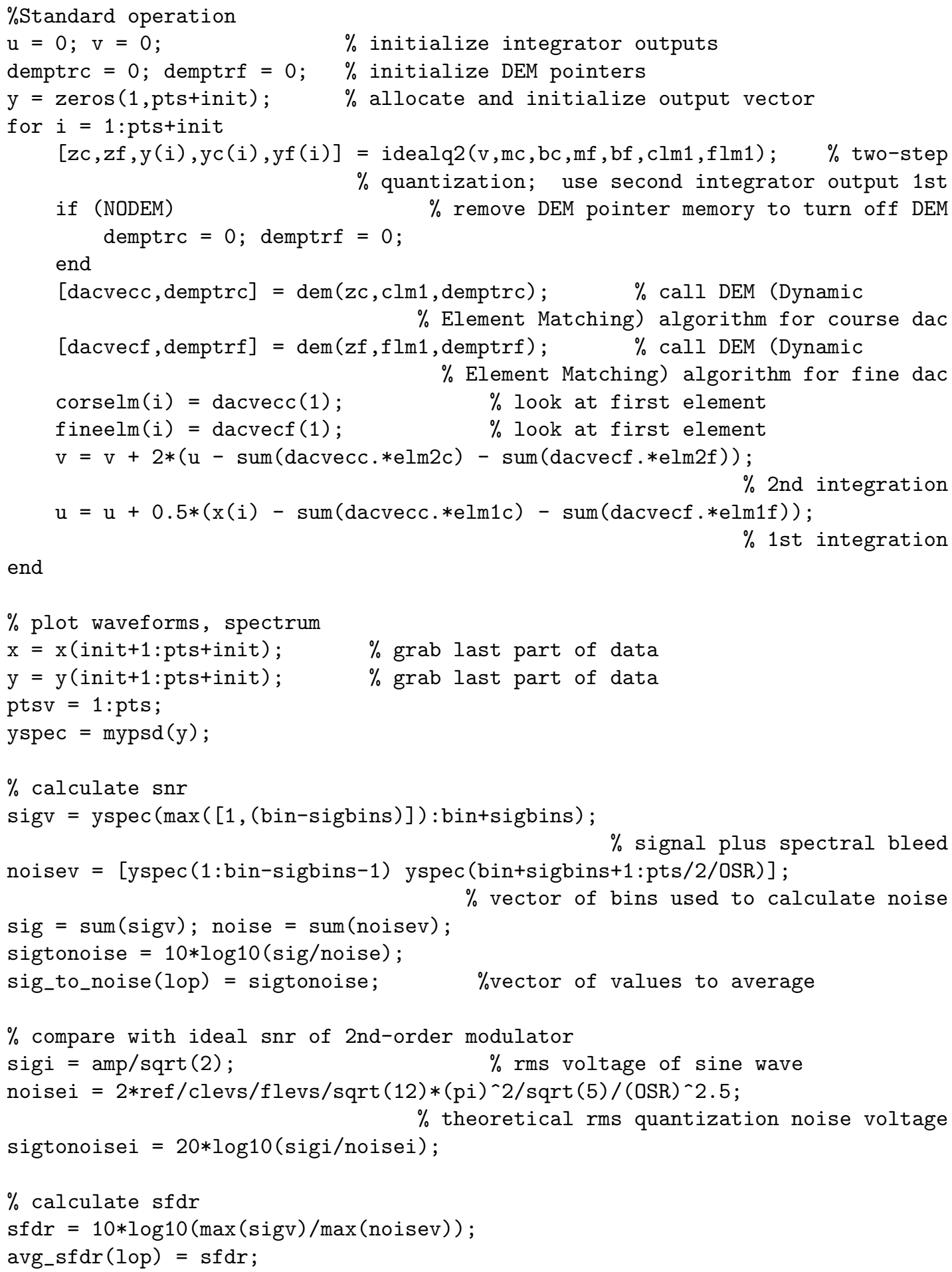




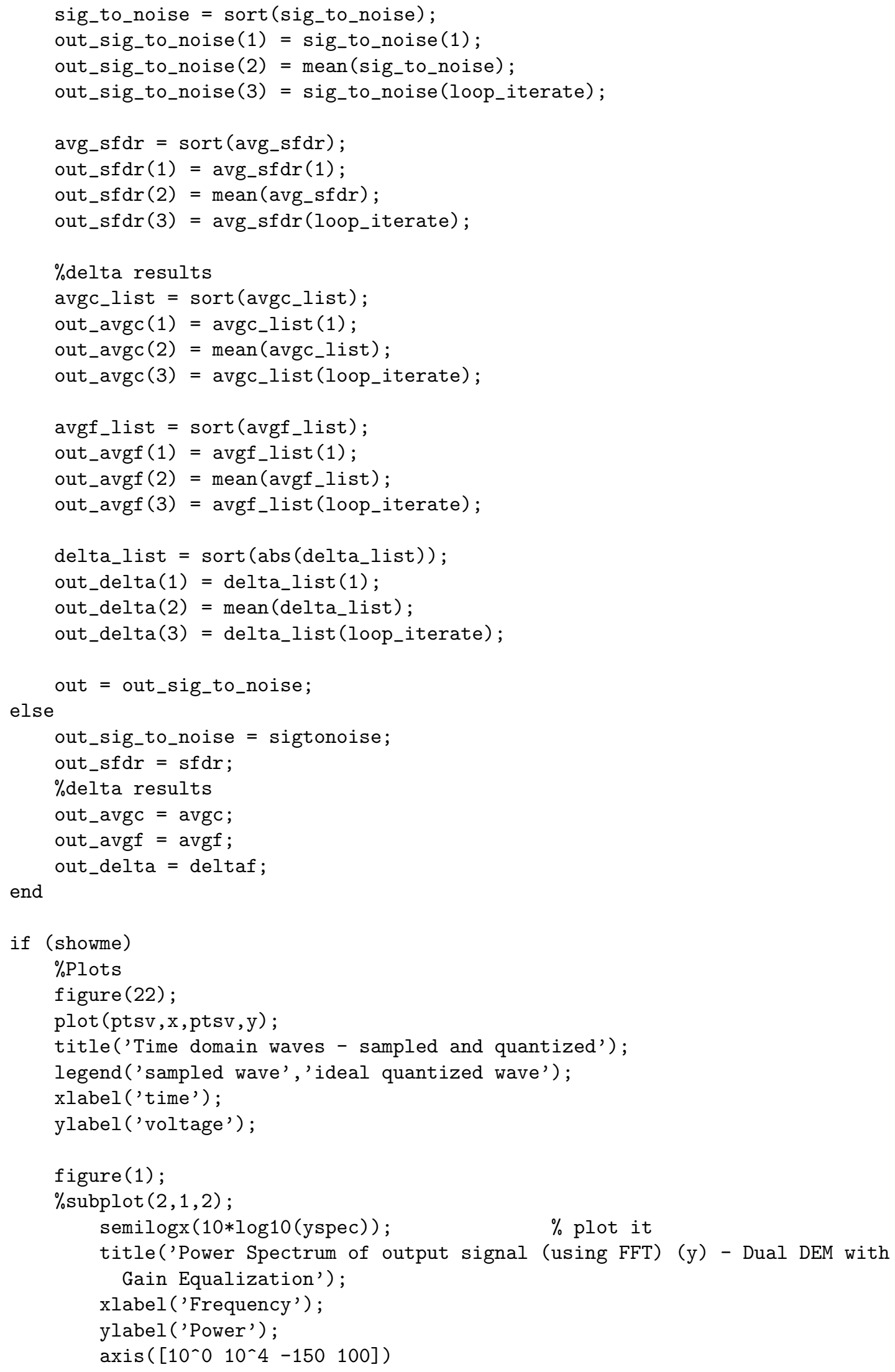




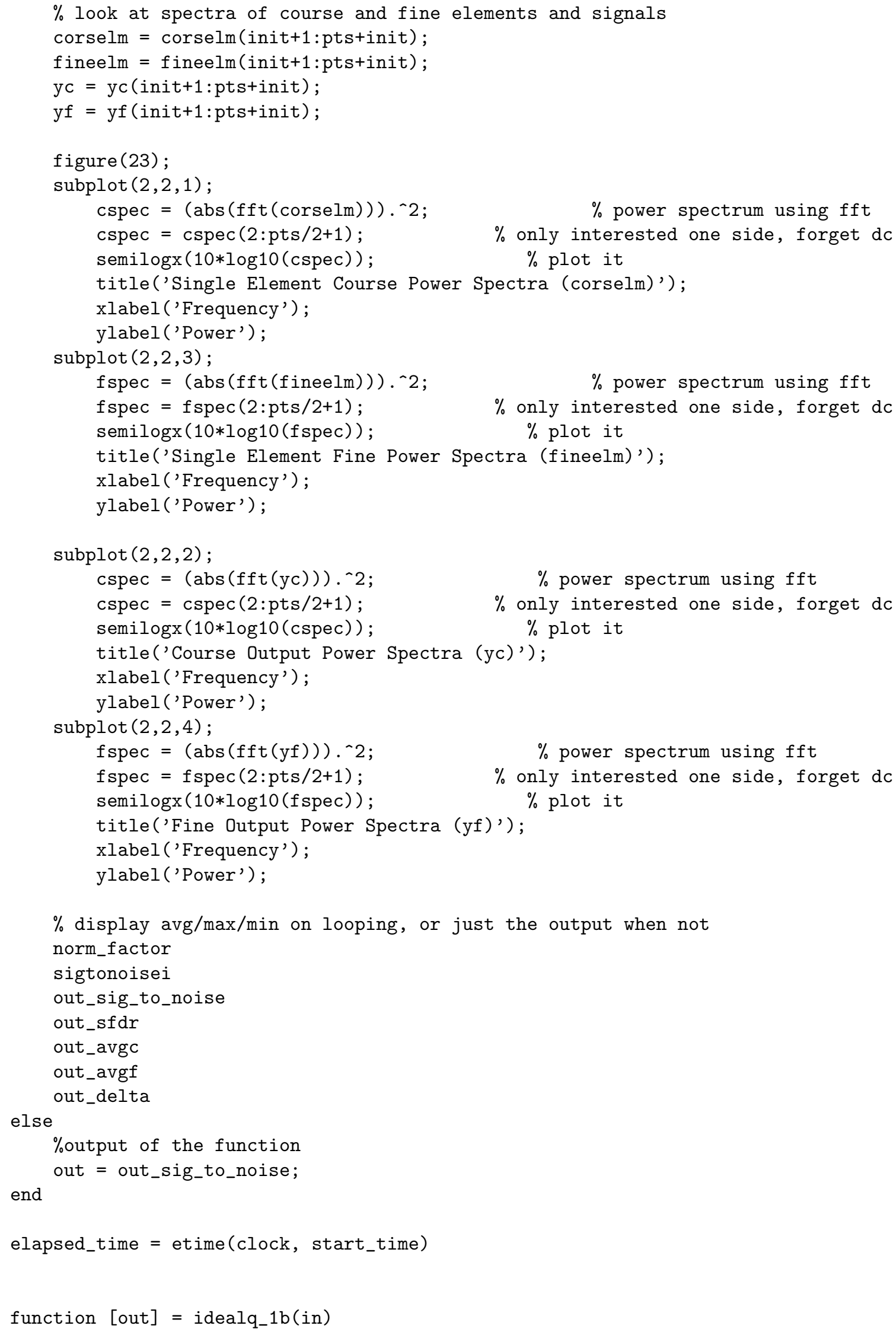




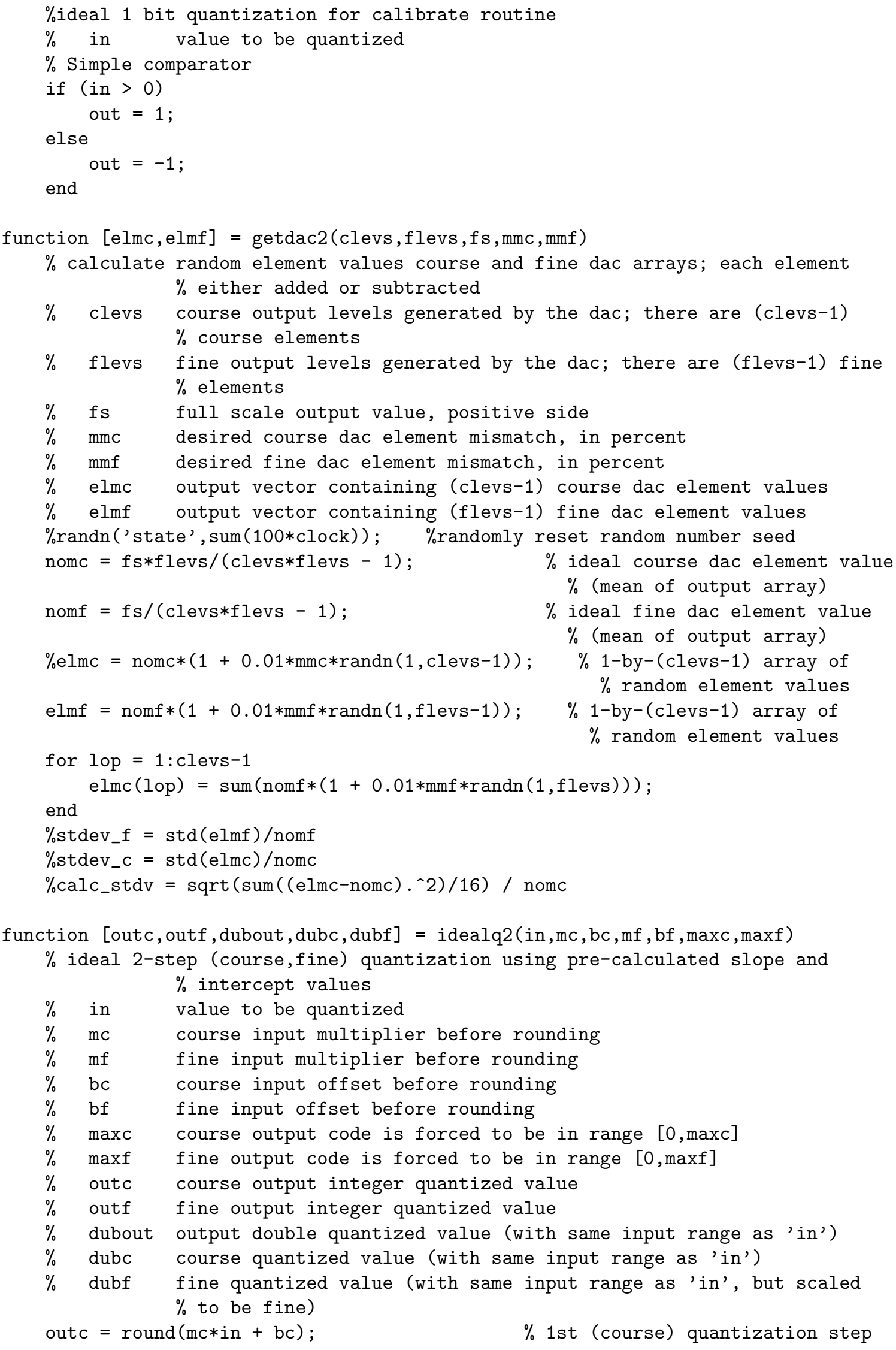




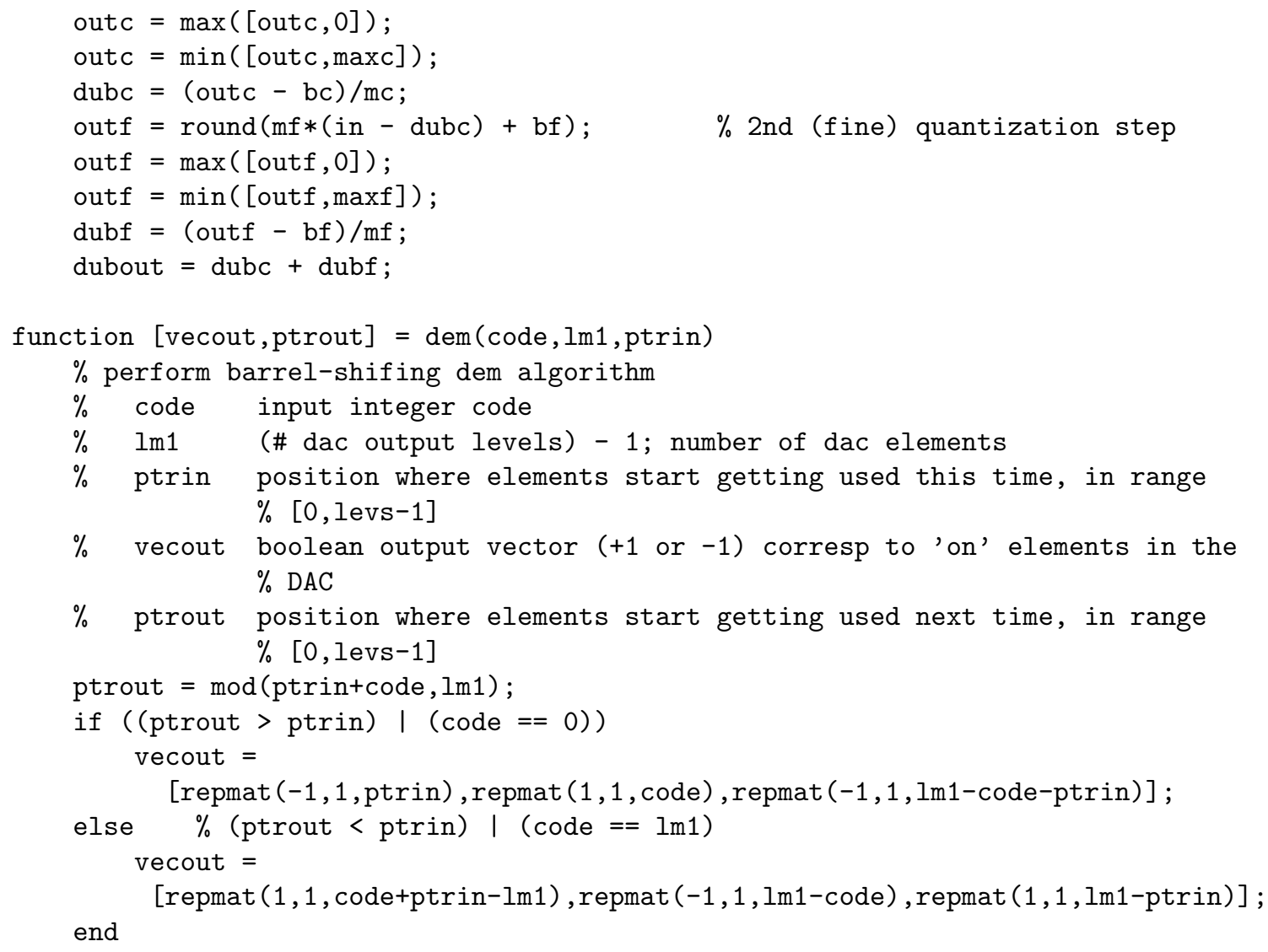




\section{B.3 ReQ Code}

function out $=$ second_order_ReQ(mm_caps, iterate)

$\%$ 2nd order 8-bit sigma-delta model and noise analysis

$\%$ include DAC mismatch and coarse/fine DEM

$\%$ Craig Petrie 6/24/2002

$\%$ Modified by Brent Nordick

start_time $=$ clock;

format long $g$

$\%$ model parameters

bin $=13 ; \quad \%$ number of sine wave cycles to capture (i.e., fft bin number)

ptspc $=1040 ; \%$ number of points per cycle

amp $=0.8 ; \quad \%$ sine wave amplitude

ref $=1.0 ; \quad \%$ reference voltage (full-scale analog input, positive side)

clevs $=16 ; \quad \%$ number of coarse levels (output codes) in two-step internal

$\%$ quantizer

flevs $=16 ; \quad \%$ number of fine levels in internal quantizer

clevs_ReQ $=16$; \% number of coarse levels (output codes) in ReQ circuitry -

$\%$ flevs_ReQ is automatically calculated

$\%$ flevs_ReQ $=32 ; \%$ number of fine levels in ReQ circuitry (total of the ReQ bits

$\%$ should be 1 extra for the overlap)

init $=4 ; \quad \%$ initialization points

OSR $=20 ; \quad \%$ oversampling ratio

dacmmc $=0 ; \quad \%$ percent mismatch $($ in $\%$ ) of coarse dac elements

dacmmf $=0 ; \quad \%$ percent mismatch (in $\%$ ) of fine dac elements

sigbins $=1 ; \quad \%$ bins on either side of signal to lump with signal

NODEM $=0 ; \quad \%$ set to 1 to turn off DEM

loop_iterate $=1 ; \%$ Make it loop to get average StoN

showme $=1 ; \quad \%$ variable to control display of values and plots

$\%$ startup as function necessary stuff

if nargin $>0$

dacmmf $=\mathrm{mm}_{-}$caps;

end

if nargin > 1

loop_iterate $=$ iterate;

end

if nargout $>0$

showme $=0$;

end

$\%$ Initial calculations

pts $=$ bin*ptspc;

norm_factor $=$ ptspc/ $(2 *$ OSR $)$;

flevs_ReQ $=2 *$ clevs $* f l e v s / c l e v s \_R e Q ;$

$\%$ calculate parameters for internal two-step adc function

$\%$ for fine quantization, input signal is in the range [-ref/clevs, +ref/clevs]

olevs = clevs*flevs; $\%$ number of overall levels in internal quantizer

olm1 = olevs - 1;

clm1 = clevs - 1;

$\mathrm{flm} 1=\mathrm{flevs}-1$; 


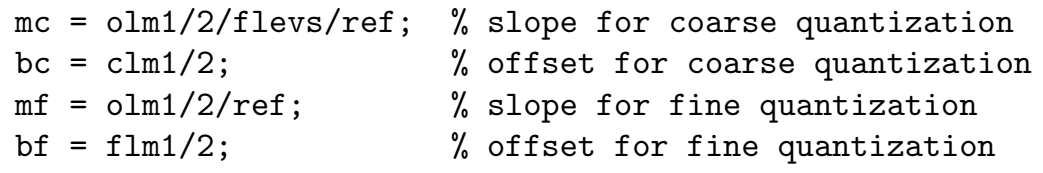




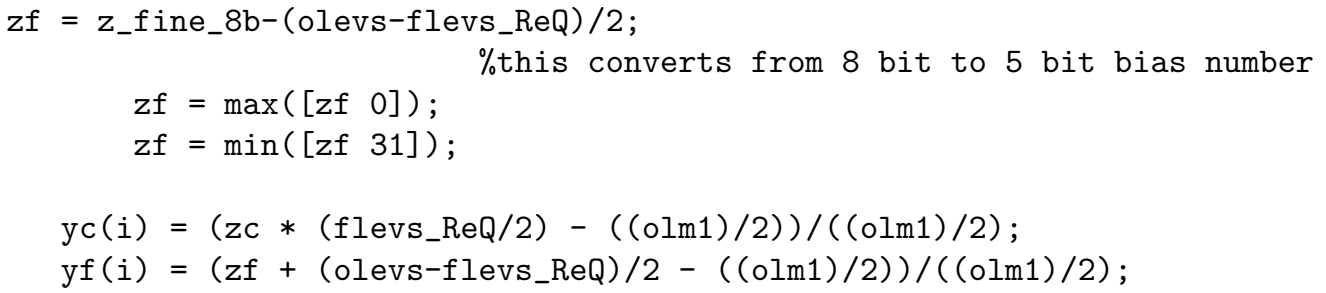

$\%$ 1st integration end

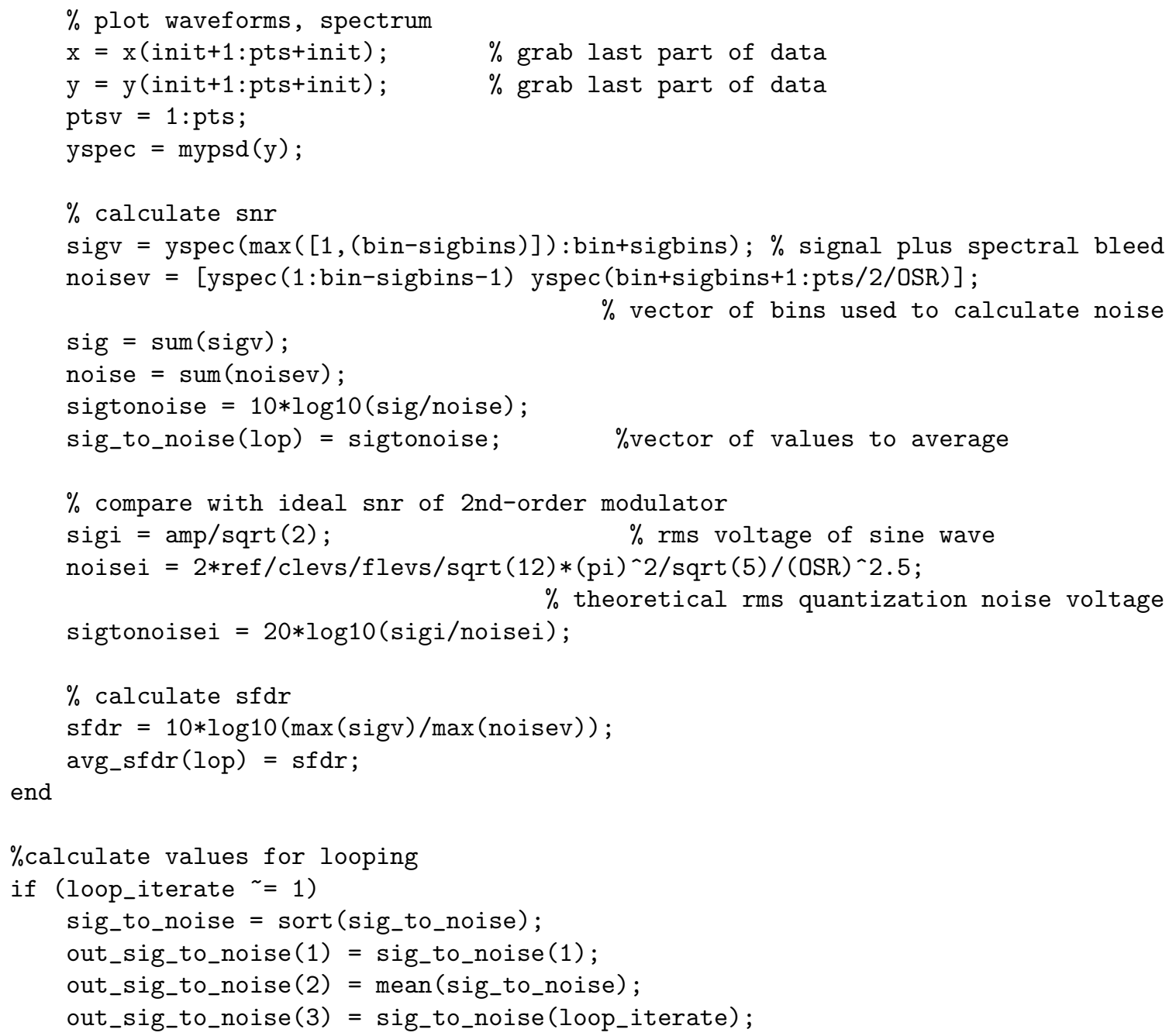




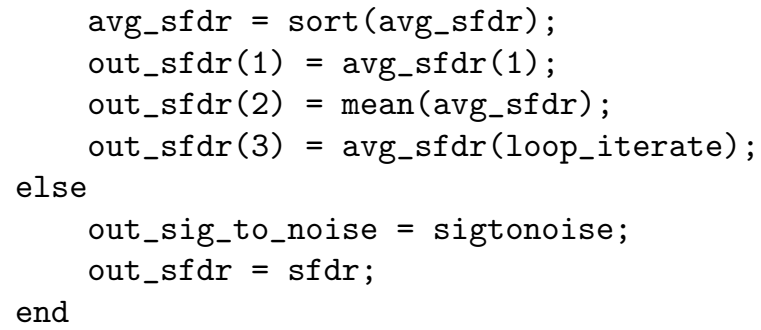




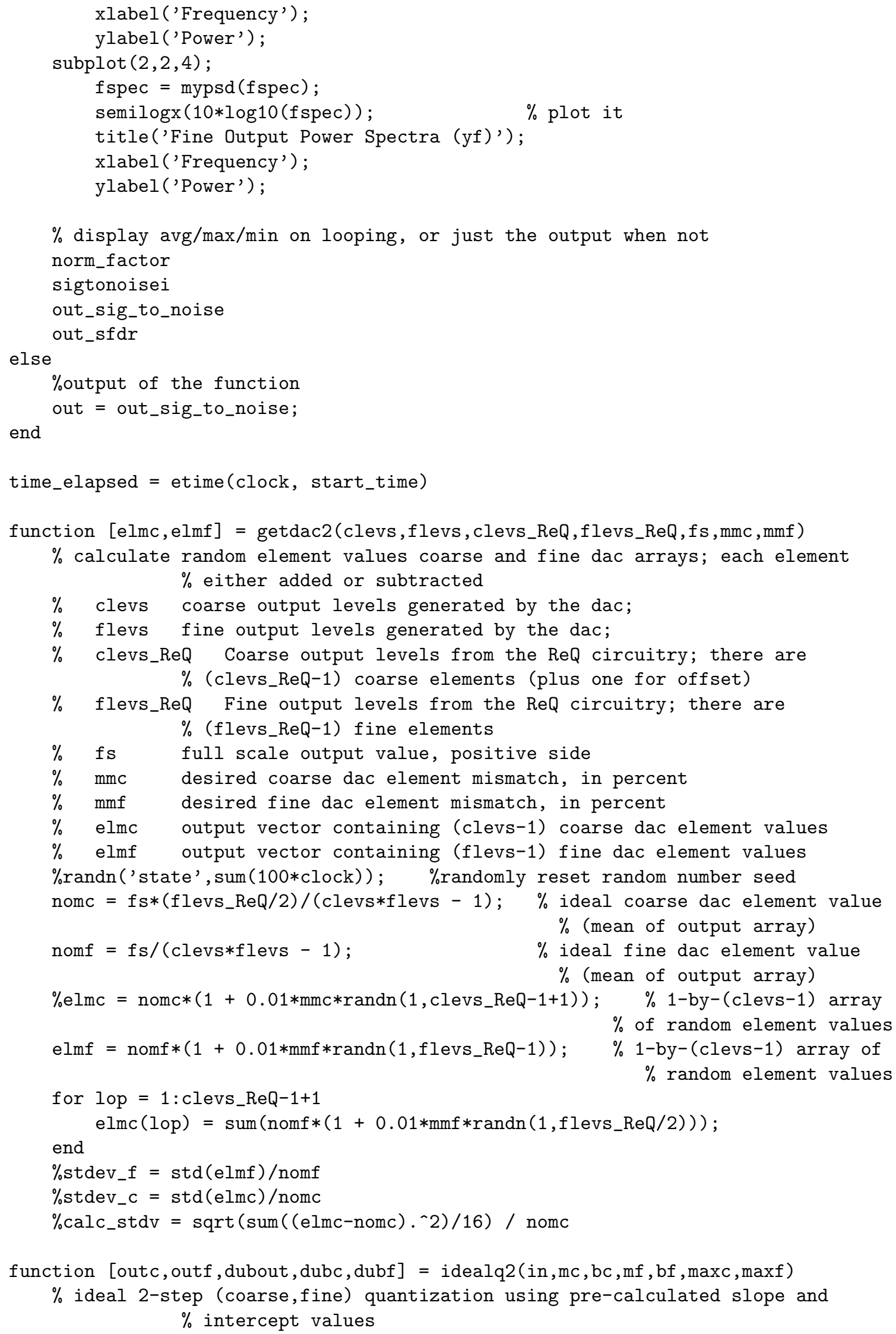




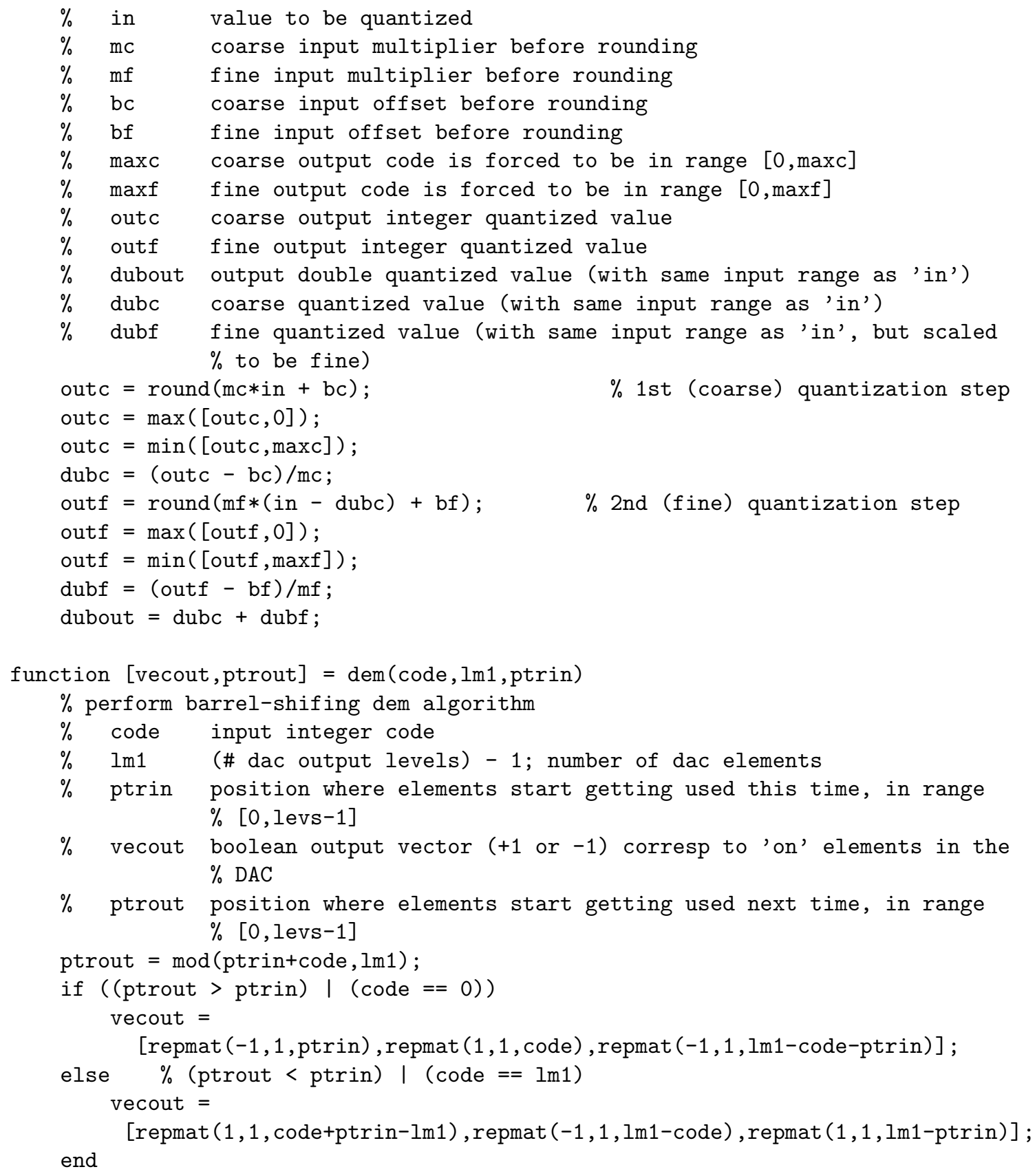




\section{B.4 Manual Calibration Code}

function out $=$ second_order_man_calibrate(mm_fine, iterate)

$\%$ 2nd order 8-bit sigma-delta model and noise analysis

$\%$ include DAC mismatch and course/fine DEM

$\%$ Craig Petrie 6/24/2002

$\%$ Modified by Brent Nordick

start_time = clock;

format long $g$

$\%$ model parameters

bin $=13 ; \quad \%$ number of sine wave cycles to capture (i.e., fft bin number)

ptspc $=1040 ; \%$ number of points per cycle

amp $=0.8 ; \quad \%$ sine wave amplitude

ref $=1.0 ; \quad \%$ reference voltage (full-scale analog input, positive side)

clevs $=16 ; \quad \%$ number of course levels (output codes) in two-step internal

$\%$ quantizer

flevs $=16 ; \quad \%$ number of fine levels in internal quantizer

init $=4 ; \quad \%$ initialization points

OSR $=20 ; \quad \%$ oversampling ratio

dacmmc $=0 ; \quad \%$ percent mismatch (in $\%$ ) of course dac elements

dacmmf $=0 ; \quad \%$ percent mismatch (in $\%$ ) of fine dac elements

sigbins $=1 ; \quad \%$ bins on either side of signal to lump with signal

NODEM $=0 ; \quad \%$ set to 1 to turn off DEM

loop_iterate $=1 ; \%$ Make it loop to get average StoN

showme $=1$; $\quad \%$ variale to control display of values and plots

$\%$ startup as function necessary stuff

if nargin $>0$

dacmmf = mm_fine;

end

if nargin $>1$

loop_iterate = iterate;

end

if nargout $>0$

showme $=0$;

end

$\%$ Initial calculations

pts = bin*ptspc;

norm_factor $=$ ptspc $/(2 * 0 S R)$;

$\%$ calculate parameters for internal two-step adc function

$\%$ for fine quantization, input signal is in the range [-ref/clevs, +ref/clevs]

olevs = clevs*flevs; $\%$ number of overall levels in internal quantizer

olm1 = olevs - 1;

$\operatorname{clm} 1=$ clevs -1 ;

$\mathrm{flm} 1=\mathrm{flevs}-1$;

$\mathrm{mc}=\mathrm{olm} 1 / 2 / \mathrm{flevs} / \mathrm{ref} ; \%$ slope for course quantization

$\mathrm{bc}=\mathrm{clm} 1 / 2 ; \quad \%$ offset for course quantization

$\mathrm{mf}=\mathrm{olm} 1 / 2 /$ ref; $\quad \%$ slope for fine quantization

$\mathrm{bf}=\mathrm{flm} 1 / 2 ; \quad \%$ offset for fine quantization 
$\%$ set up loop for averaging purposes

for lop $=1:$ loop_iterate

$$
\begin{aligned}
& \mathrm{x}=\operatorname{amp} * \sin (2 * \mathrm{pi} * \mathrm{bin} *[0: \mathrm{pts}-1+\mathrm{init}] / \mathrm{pts}) ; \quad \% \text { exactly 'bin' cycles of } \\
& \% \text { 'pts'-point sine wave } \\
& {[e \operatorname{lm} 1 c, e \operatorname{lm} 1 \mathrm{f}]=\operatorname{getdac} 2(\mathrm{clevs}, \mathrm{flevs}, \mathrm{ref}, \mathrm{dacmmc}, \mathrm{dacmmf}) ; \quad \% \text { retrieve dac }} \\
& \% \text { element values for dac \#1 } \\
& {[e l m 2 c, e l m 2 f]=\operatorname{getdac} 2(c l e v s, f l e v s, r e f, 0,0) ;} \\
& \% \text { retrieve dac } \\
& \% \text { element values for dac \#2 }
\end{aligned}
$$

$\%$ compute averages and adjust fine values so that the average is the same

$\%$ this translates to same average gain

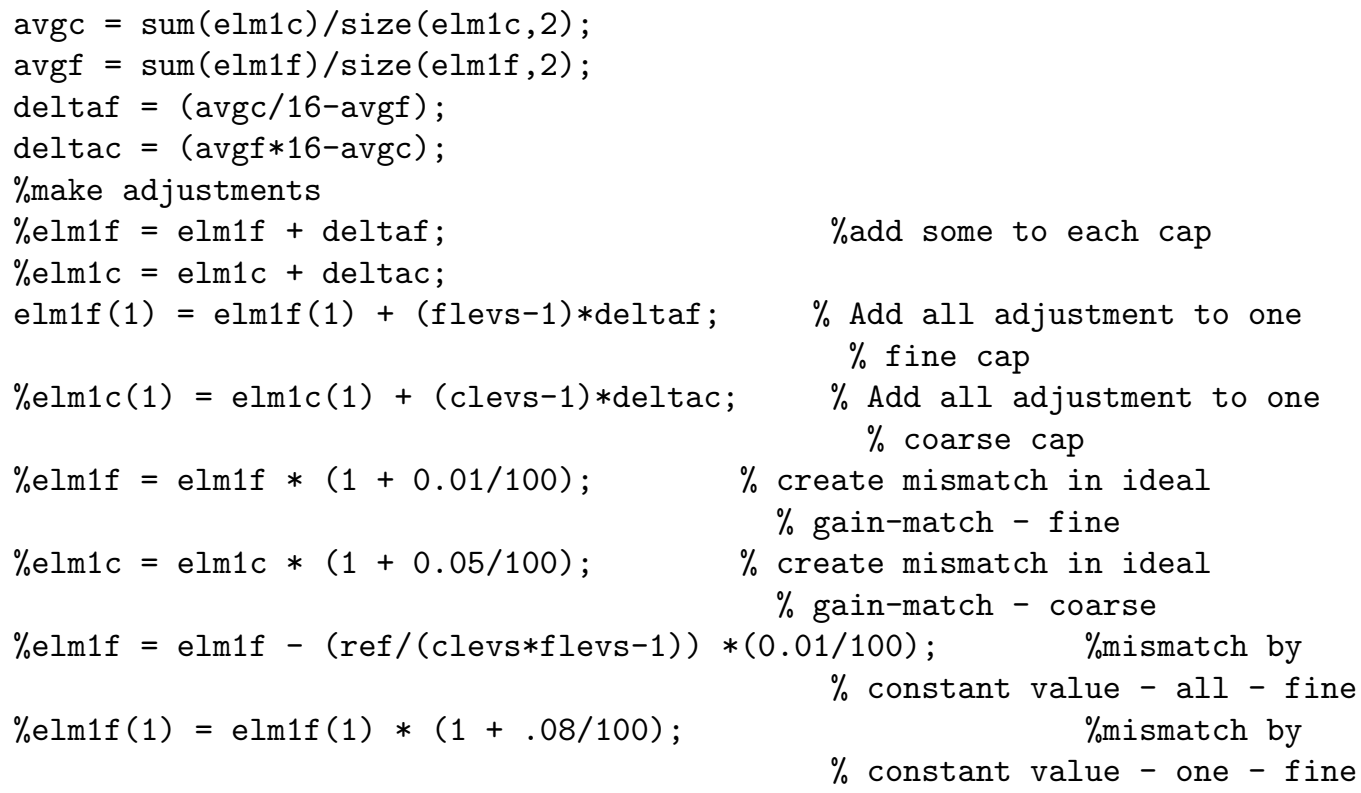




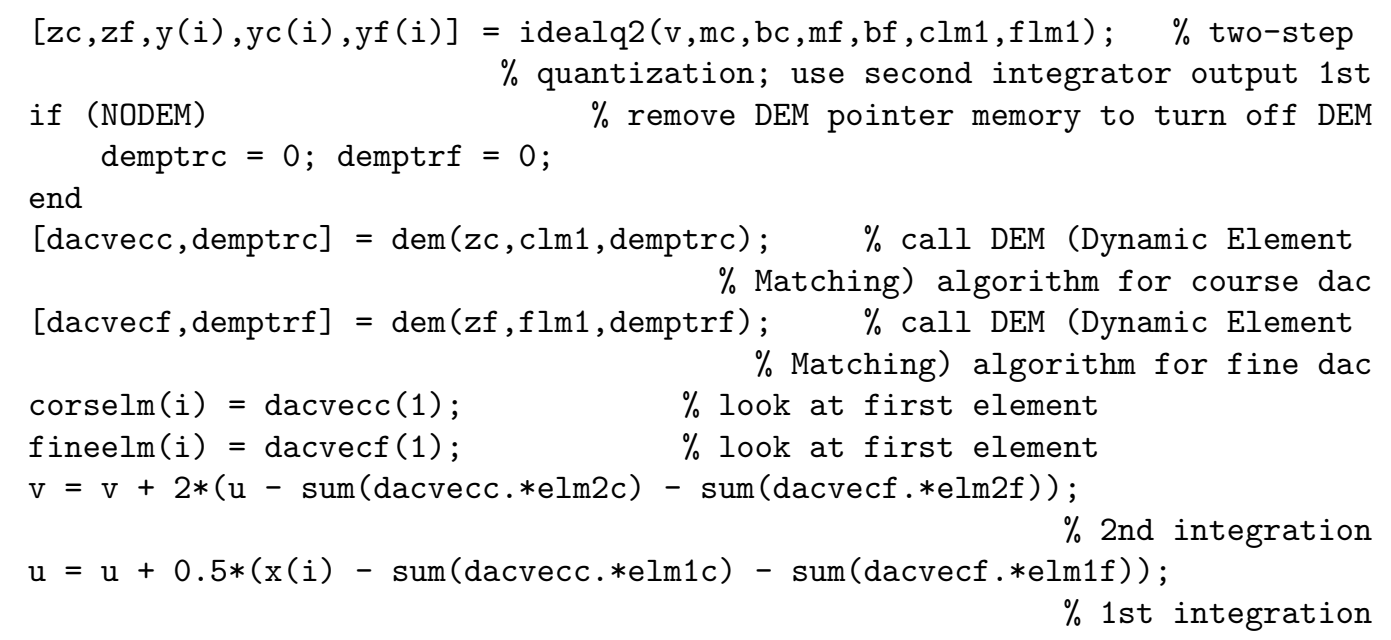
end

$\%$ plot waveforms, spectrum $\mathrm{x}=\mathrm{x}$ (init+1:pts+init); $\quad \%$ grab last part of data

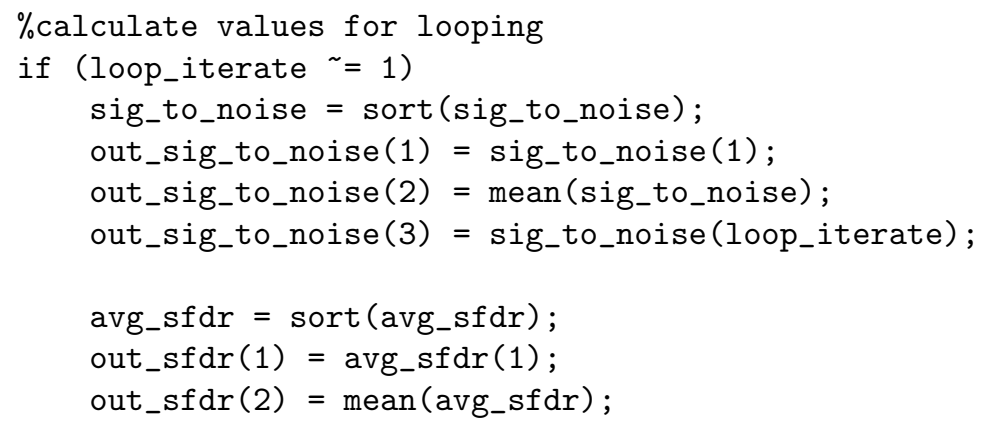




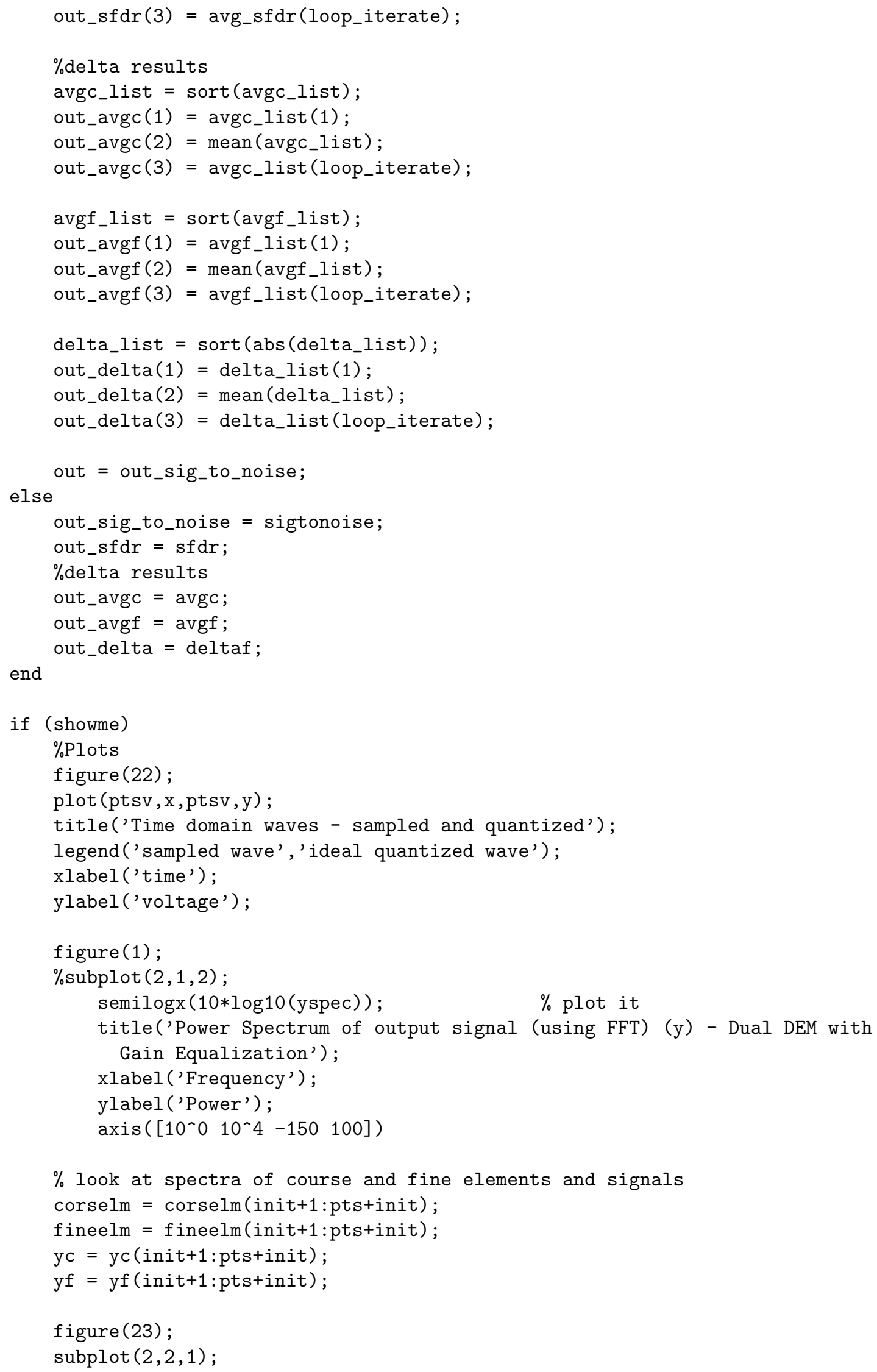




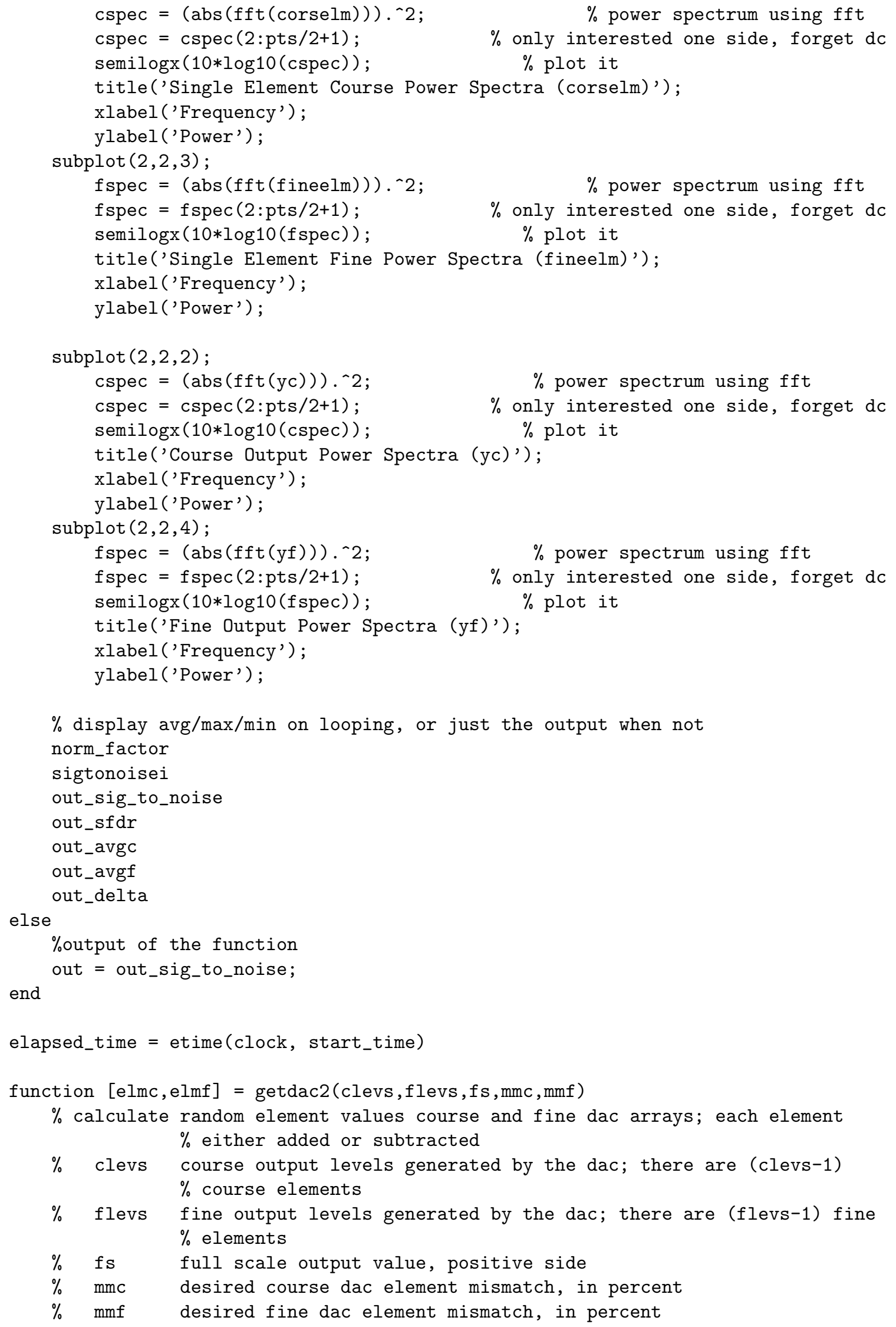




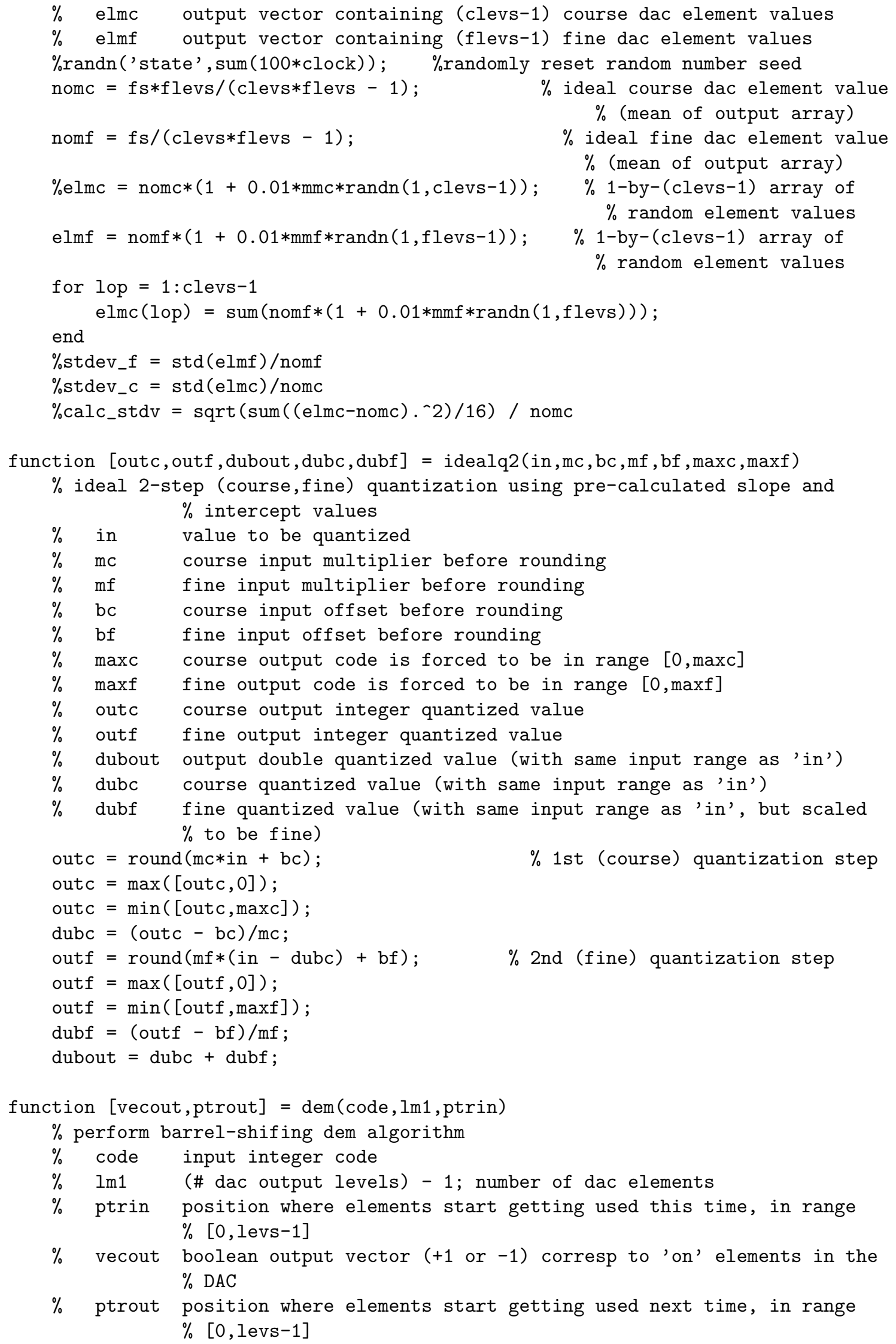




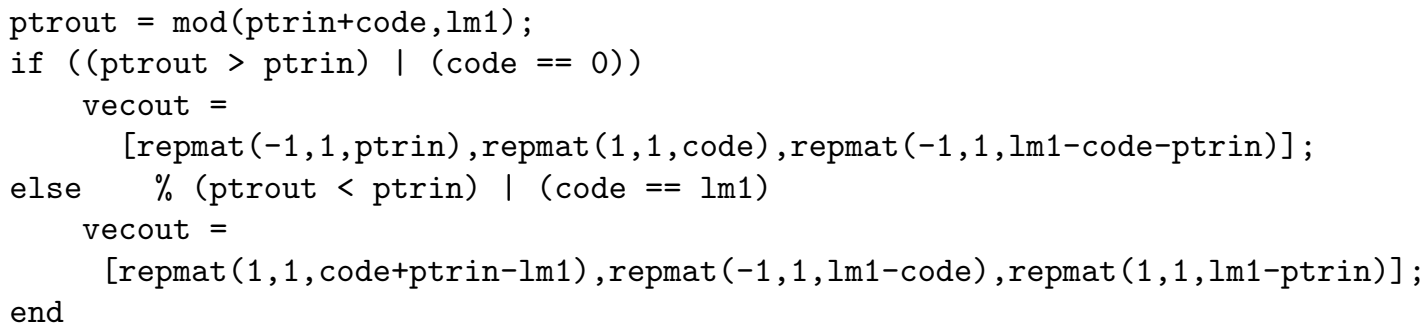




\section{B.5 Additional Functions}

\section{B.5.1 mypsd}

function out $=\operatorname{mypsd}($ signal)

$\%$ Attempt to create a "mypsd" function

$\%$ Brent Nordick 01/17/03

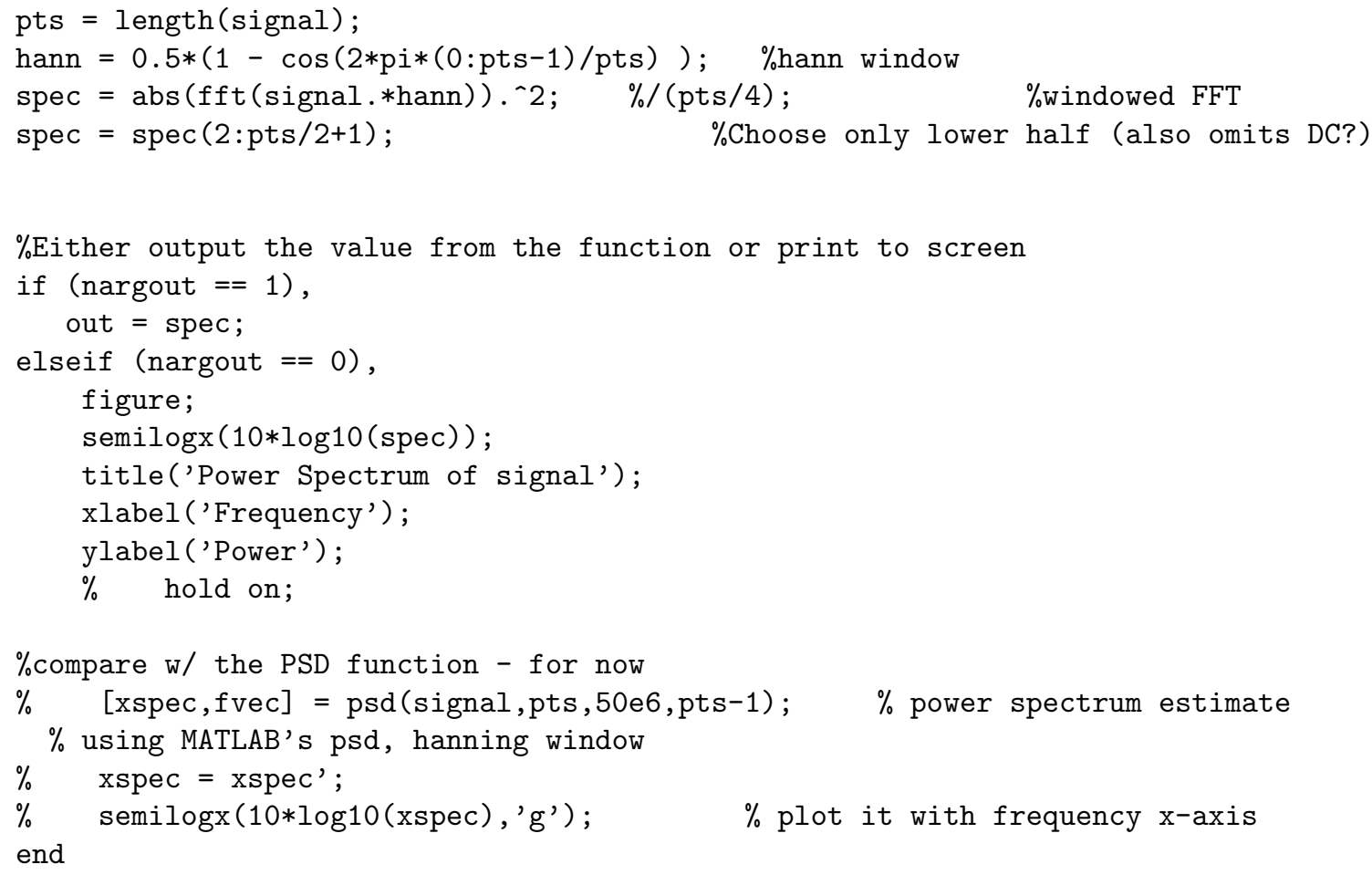




\section{B.5.2 mysnr}

function out $=$ mysnr (signal, bin, OSR)

$\%$ Attempt to create a "mysnr" function to calculate the signal-to-noise ratio $\%$ of a given signal

$\%$ Brent Nordick 01/22/03

$\%$ signal: the signal to be analized

\% bin: number of bins to divide the signal into

\%temp or needed to pass?

sigbins = 1 ;

pts $=\operatorname{size}($ signal $)$;

sigv $=\operatorname{signal}(\max ([1,($ bin-sigbins $)]):$ bin+sigbins $) ; \quad \%$ signal plus spectral bleed noisev $=[$ signal $(1$ : bin-sigbins-1) signal (bin+sigbins+1:pts (2)/OSR) $]$

$\%$ vector of bins used to calculate noise

sig $=\operatorname{sum}($ sigv $)$;

$\%$ aparently wants pts/2/OSR to be an integer

noise $=\operatorname{sum}($ noisev);

sigtonoise $=10 * \log 10(\mathrm{sig} /$ noise $)$;

$\%$ Either output the value from the function or print to screen

if (nargout $==1$ ), out = sigtonoise;

elseif (nargout $==0$ ), disp ('SNR');

disp (sigtonoise);

end 


\section{Bibliography}

[1] S. R. Norsworthy, R. Schreier, and G. C. Temes, Delta-Sigma Data Converters: Theory, Design, and Simulation, IEEE Press, New York, 1997.

[2] e. a. D. R. Welland, "A stereo 16 bit delta-sigma A/D converter for digital audio", in J. Audio Eng. Soc, 1989, vol. 37, pp. 476-485.

[3] K. Yamamura, A. Nogi, and A. Barlow, "A low power 20 bit instrumentation deltasigma ADC", in Proceedings of the IEEE 1994 Custom Integrated Circuits Conferenc, 1994, pp. 23.7.1-23.7.4.

[4] Y. Geerts, A. M. Marques, M. S. J. Steyaert, and W. Sansen, "A 3.3-v, 15-bit, deltasigma ADC with a signal bandwidth of $1.1 \mathrm{MHz}$ for ADSL applications", in IEEE Journal of Solid-State Circuits, Jul. 1999, vol. 34, pp. 927-936.

[5] R. Jiang and T. S. Fiez, "A 1.8v 14b delta-sigma A/D converter with 4Msamples/s conversion,", in Digest of Technical Papers, IEEE 2002 International Solid-State Circuits Conference (ISSCC), Feb. 2002, vol. 31, pp. 220-461.

[6] R. Adams, K. Nguyen, and K. Sweetland, "A 113-dB SNR oversampling DAC with segmented noise-shaped scrambling", in IEEE Journal of Solid-State Circuits, Dec. 1998, pp. 1871-1878.

[7] H. Stark and J. W. Woods, Probability and Random Processes with Applications to Signal Processing, Prentice Hall, Upper Saddle River, New Jersey, third edition, 2002.

[8] R. T. Baird and T. S. Fiez, "Linearity enhancement of multibit delta-sigma A/D and D/A converters using data weighted averaging", in IEEE Trans. Circuits Syst. II, Dec. 1995, vol. 42, pp. $753-762$. 
[9] O. Nys and R. K. Henderson, "A 19-bit low-power multibit sigma-delta ADC based on data weighted averaging", in IEEE Journal of Solid State Circuits, July 1997, vol. 32, pp. 933-942.

[10] S. Lindfors and K. A. I. Halonen, "Two-step quantization in multibit delta-sigma modulators", in IEEE Trans. Circuits Syst. II, Feb. 2001, vol. 48, pp. 171-176.

[11] Y. Cheng, C. Petrie, and B. Nordick, "A 4th-order single-loop delta-sigma ADC with 8-bit two-step flash quantization", in Accepted to Proc. 2004 IEEE International Symposium on Circuits and Systems (ISCAS), May 2004.

[12] A. Fishov, E. Siragusa, J. Welz, E. Fogleman, and I. Galton, "Segmented mismatchshaping D/A conversion", in Proc. 2002 IEEE International Symposium on Circuits and Systems (ISCAS), May 2002, vol. 4, pp. IV-679-IV-682.

[13] M. R. Miller and C. S. Petrie, "A multibit sigma-delta ADC for multimode receivers", in IEEE J. Solid-State Circuits, Mar. 2003, vol. 38, pp. 475-482.

[14] C. Petrie and M. R. Miller, "A background calibration technique for multibit delta-sigma modulators", in Proc. 2000 IEEE International Symposium on Circuits and Systems (ISCAS), May 2000, vol. 2, pp. 29-32.

[15] B. Nordick, C. Petrie, and Y. Cheng, "Dynamic element matching techniques for deltasigma ADCs with large internal quantizers", in Accepted to Proc. 2004 IEEE International Symposium on Circuits and Systems (ISCAS), May 2004. 